\title{
Graphical calculus of volume, inverse volume and Hamiltonian operators in loop quantum gravity
}

\author{
Jinsong Yang ${ }^{1,2, a}$, Yongge $\mathbf{M a}^{3, b}$ \\ ${ }^{1}$ Department of Physics, Guizhou University, Guiyang 550025, China \\ 2 Institute of Physics, Academia Sinica, Taipei 115, Taiwan \\ ${ }^{3}$ Department of Physics, Beijing Normal University, Beijing 100875, China
}

Received: 24 February 2016 / Accepted: 25 February 2017 / Published online: 12 April 2017

(C) The Author(s) 2017. This article is an open access publication

\begin{abstract}
To adopt a practical method to calculate the action of geometrical operators on quantum states is a crucial task in loop quantum gravity. In this paper, the graphical calculus based on the original Brink graphical method is applied to loop quantum gravity along the line of previous work. The graphical method provides a very powerful technique for simplifying complicated calculations. The closed formula of the volume operator and the actions of the Euclidean Hamiltonian constraint operator and the so-called inverse volume operator on spin-network states with trivalent vertices are derived via the graphical method. By employing suitable and non-ambiguous graphs to represent the action of operators as well as the spin-network states, we use the simple rules of transforming graphs to obtain the resulting formula. Comparing with the complicated algebraic derivation in some literature, our procedure is more concise, intuitive and visual. The resulting matrix elements of the volume operator is compact and uniform, fitting for both gauge-invariant and gauge-variant spin-network states. Our results indicate some corrections to the existing results for the Hamiltonian operator and inverse volume operator in the literature.
\end{abstract}

\section{Introduction}

As a non-perturbative approach to quantum gravity, loop quantum gravity (LQG) has made considerable achievements (see $[1,2]$ for review articles, and [3,4] for books). This theory rigorously enforces the lesson of general relativity and is built on a strict mathematical foundation. In LQG, the quantum kinematical Hilbert space $\mathcal{H}_{\text {kin }}$ was successfully constructed with the spin-network states as its orthonormal basis. The elementary operators are the holonomy and flux operators. By suitable regularization schemes, quantum geometric operators, such as the length, area, and volume operators corresponding to their classical quantities, were well defined on $\mathcal{H}_{\text {kin }}$ [5-11]. The volume operator is a cornerstone on which some physical interesting operators, for instance, the Hamiltonian constraint operator determining the quantum dynamics of LQG, can be constructed.

It is well known that quantum dynamics is a central issue in LQG. There are two main approaches to the quantum dynamics, based on the canonical and covariant quantization programs, respectively. In canonical quantization, the quantum dynamics is determined by some quantum Hamiltonian constraint operator. In the covariant program the quantum dynamics is to define a reasonable transition amplitude. One expects that the quantum dynamics from the two different approaches can make same physical predictions. Such an expectation has been achieved at least in 3-dimensional LQG to certain sense [12]. Although some progress has been made for 4-dimensional case in checking the consistency between the two approaches [13-15], the issue is not yet understood up to now. To understand the relation between the canonical and covariant quantum dynamics, we not only need a suitable definition of the Hamiltonian constraint operator, but also have to calculate its matrix elements on given quantum states. In the light of the seminal work by Thiemann [16,17], some mathematically well-defined Hamiltonian constraint operators have been constructed in LQG. All of these Hamiltonian operators are defined by the volume operator. There are two versions of the volume operator in the literature. The first one, based on "external" regularization scheme, was introduced by Rovelli and Smolin in loop representation [8] and re-obtained in the connection representation [10]. The

\footnotetext{
${ }^{a}$ e-mail: yangksong@gmail.com

be-mail: mayg@bnu.edu.cn
} 
second one, based on the "internal" regularization, was firstly defined by Ashtekar and Lewandowski [10]. In [11], Thiemann presented a rather short and compact regularization procedure to re-derive the second version of the volume operator. Playing a crucial role in LQG, the spectra of the volume operator is pursued. Certain matrix elements of the volume operator were calculated in the framework of loop representation by using a graphical tangle-theoretic Temperley-Lieb formulation in [18]. Then they were also derived in connection representation by a rigorous but tedious algebraic method in [11,19], and their special case was re-derived using generalized Wigner-Eckart theory [20] as well as the graphical methods in [13,21-23]. Although those components of the volume operator are rigorously defined, the computation of their actions on spin-network states are difficult. The main reason is the following. The volume element operator at a vertex $v$ of a graph $\gamma$ reads $V_{v}=\sqrt{\left|\hat{q}_{v}\right|}$. Although the matrix elements of $\hat{q}_{v}$ can be calculated using recoupling theory, the matrix has no obvious symmetries and hence is difficult to diagonalize analytically for the case that the dimension of the matrix is bigger than nine. On the one hand, the derivation of closed formula in [19] is rigorous. But there is no universal formula with so tedious and abstract method. Thanks to the calculation of the matrix elements of the volume operator in certain special case, some matrix elements of Thiemann's Hamiltonian constraint operator and its generalization were derived in [21,22]. Later on, the matrix elements was re-derived in [13], and then the formula in [13] was corrected by sign factors in [14,23] using graphical method. Matter coupling is also an important issue in LQG. In the case of gravity coupled to a scalar field, the whole Hamiltonian constraint operator was constructed [17,24]. The matter part of the whole Hamiltonian constraint operator usually contains the "inverse volume operator", which is defined by the co-triad operators. In the symmetric model of loop quantum cosmology (LQC) [25], the analog of the inverse volume operator is bounded above. This fact is sometimes thought of as a reason for the singularity resolution in LQC. In particular, it is shown in [26] that in spatially curved anisotropic models inverse volume effects may become important to bind expansion and shear scalars. However, it is shown in [27] that the inverse volume operator with certain ordering in full LQG is unbounded on the zero volume eigenstates (at a gauge-invariant trivalent vertex). This throws doubt on whether one can generalize the conclusions of LQC to LQG. To understand definitely the inverse volume operator in LQG and its relation to the analogs in certain symmetric models, it is necessary to calculate in detail its action on the quantum states in LQG. There is no doubt that a simple and practical calculation method is desirable to further understand the volume, inverse volume and Hamiltonian constraint operators.

As a powerful tool for practical calculation, graphic calculus has been introduced in LQG in a few papers (see e.g., $[13,14,18,21-23,28,29])$. These graphical methods are based on the graphical methods developed by Yutsis in [30], Brink in [31], Varshalovich in [32], and Kauffman in [33]. In order to represent conveniently the Clebsch-Gordan coefficients, Brink slightly modified the Yutsis' graphical method by introducing a line with an arrow on it to represent "metric tensor". Comparing to the Yutsis' method, Brink's graphical method is more convenient and has wider scope of application. Varshalovich's method gives a way to represent the Dirac's bra and ket notation by introducing a line with an arrow outgoing from a node to represent "ket" (state vector) and a line with double arrow coming into a node to represent the "bra" (dual state vector). The above three methods are usually used to deal with the coupling problem of angular momentum in quantum mechanics. Moreover, Kauffman introduced a graphical method for the Temperley-Lieb algebra. Kauffman's graphical method was firstly used in LQG in $[18,21,22]$. It is worth noting that Kauffman's method was in fact used in [13] while the graphical notations in its main text are similar to those in [22]. Brink's graphical method was only recalled in the appendix of [13]. Then Varshalovich's method was adopted in $[14,23,28]$. Brink's graphical method was also taken to study the propagator of spinfoam models in [29], in which the graphical method was only used to calculate the action of the right-invariant vector field (the "grasping operator") on the intertwiners but not the action of holonomy operator. Graphical method was also introduced to quantum reduced gravity in [34]. In this paper, the graphical calculus based on the Brink graphical method [31] and its suitable extension ${ }^{1}$ will be employed to study the volume, inverse volume and Hamiltonian constraint operators in LQG. Our aim is in two folds. One is to show that the graphical method is suitable to calculate the actions of different kinds of operators on spin-network states. The other is to cross-check the results obtained in the literature, on which some important applications are based. This method consists of two ingredients, graphical representation and graphical calculation. The algebraic formula will be represented by corresponding graphical formula in an unique and unambiguous way. Then the graphical calculation will be performed following the simple rules of transforming graphs, corresponding uniquely to the algebraic manipulation of the formula. A central goal of this paper is to derivate the closed formula for the matrix element of the volume operator, which involves only the flux operator, based on the rigorous graphical method. Comparing to the algebraic method, our derivation is obviously more compact and simple. Our analysis shows that the formula of the matrix elements for certain cases in [19] is also valid for other cases and hence can be regarded as a general expression. Then we will consider the actions of the gravitational Hamiltonian constraint operator and the inverse volume operator on spin-network states in the graphical

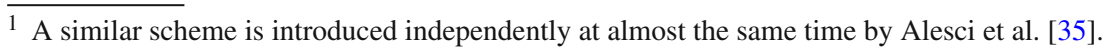


method. Both operators depend also on the honolomies in addition to fluxes. Note that, besides the regularization introduced by Thiemann [16], other proposal for the regularization of the Hamiltonian constraint operator is also available [36]. On the contrary to the conclusion in [27], our calculation shows that the inverse volume operator is bounded (zero-valued) at a trivalent non-planar vertex of the gauge-invariant spin-network states, which is a non-trivial eigenstate with zero-eigenvalue of the volume operator. This result opens a possible way to lift the result of singularity resolution of LQC to LQG.

This paper is organized as follows. Section 2 is devoted to a brief review of the elements of LQG. In Sect. 3, the graphical method to LQG will be introduced systematically. In Sect. 4, we will derive the closed formula for the matrix element of the volume operator by the graphical method. It is shown how the simple rules of transforming graphs tremendously simplify our calculation. In Sect. 5, the construction of Thiemann's Euclidean Hamiltonian constraint operator will be briefly reviewed, and its action on gauge invariant trivalent spin-network states will be calculated by the graphical method. In Sect. 6, we will compute the action of the inverse volume operator appeared in the Hamiltonian constraint for gravity coupled to matter field. The results will be discussed in Sect. 7. In Appendix A, we will review the representation theory of $S U(2)$ group, including the notation of intertwiners and basic components of Brink's graphical representation and some rules of transforming graphs. The detailed proof of some identities and results in the main text will be presented in Appendix B and Appendix C separately.

\section{Preliminaries}

In this section, we briefly summarize the elements of LQG to establish our notations and conventions (see [1-4] for details). The classical starting point of LQG is the Hamiltonian formalism of GR, formulated on a 3-dimensional manifold $\Sigma$ of arbitrary topology. With Ashtekar-Barbero variables [37,38], GR can be cast in the form of a dynamical theory of the connection with $S U(2)$ gauge group. We denote spatial indices by $a, b, c, \ldots$ and internal indices by $i, j, k, \ldots=1,2,3$. The phase space consists of canonical pairs $\left(A_{a}^{i}, \tilde{E}_{i}^{a}\right)$ of fields on $\Sigma$, where $A_{a}^{i}$ is a connection 1-form which takes values in the Lie algebra $s u(2)$, and $\tilde{E}_{i}^{a}$ is a vector density of weight 1 . The densitized triad $\tilde{E}_{i}^{a}$ is related to the co-triad $e_{a}^{i}$ by $\tilde{E}_{i}^{a}:=\frac{1}{2} \tilde{\epsilon}^{a b c} \epsilon_{i j k} e_{b}^{j} e_{c}^{k} \operatorname{sgn}\left(\operatorname{det}\left(e_{d}^{l}\right)\right)$, where $\tilde{\epsilon}^{a b c}$ is the Levi-Cività tensor density of weight 1 , and $\operatorname{sgn}\left(\operatorname{det}\left(e_{d}^{l}\right)\right)$ denotes the sign of $\operatorname{det}\left(e_{d}^{l}\right)$. The 3-metric on $\Sigma$ is expressed in terms of co-triads through $q_{a b}=e_{a}^{i} e_{b}^{j} \delta_{i j}$. The only non-trivial Poisson bracket reads

$\left\{A_{a}^{i}(x), \tilde{E}_{j}^{b}(y)\right\}=\kappa \beta \delta_{a}^{b} \delta_{j}^{i} \delta^{3}(x, y)$,

where $\kappa=8 \pi G$, and $\beta$ is the Barbero-Immirzi parameter. The behavior of the connection under finite gauge transformations is

$A \mapsto A^{g}=-(\mathrm{d} g) g^{-1}+g A g^{-1}$.

The fundamental variables in LQG are the holonomy of the connection along a curve and the flux of densitized triad through a 2-surface. Given an edge $e:[0,1] \rightarrow \Sigma$, the holonomy $h_{e}(A)$ of connection $A_{a}^{i}$ along the edge $e$ is

$h_{e}(A):=\mathcal{P} \exp \left(\int_{e} A\right)=\mathbb{I}_{2}+\sum_{n=1}^{\infty} \int_{0}^{1} \mathrm{~d} t_{1} \times \int_{t_{1}}^{1} \mathrm{~d} t_{2} \cdots \int_{t_{n-1}}^{1} \mathrm{~d} t_{n} A\left(e\left(t_{1}\right)\right) \cdots A\left(e\left(t_{n}\right)\right)$,

where $A(e(t)):=\dot{e}^{a}(t) A_{a}^{i}(e(t)) \tau_{i}$, with $\dot{e}^{a}(t)$ being the tangent vector of $e$, and $\tau_{i}:=-i \sigma_{i} / 2$ (with $\sigma_{i}$ being the Pauli matrices), $\mathcal{P}$ denotes the path ordering which orders the smallest path parameter to the left. The holonomy $h_{e}(A)$ is the unique solution $h_{e([0, t=1])}(A)$ of the parallel transport equation

$\frac{\mathrm{d} h_{e([0, t])}(A)}{\mathrm{d} t}=h_{e([0, t])}(A) A(e(t))$

with the initial value $h_{e([0,0])}(A)=\mathbb{I}_{2}$. Define a combination $\circ$ of two edges $e_{1}, e_{2}$ satisfying $e_{1}(1)=e_{2}(0)$ as

$\left[e_{1} \circ e_{2}\right](t):=\left\{\begin{array}{ll}e_{1}(2 t), & t \in\left[0, \frac{1}{2}\right] \\ e_{2}(2 t-1), & t \in\left[\frac{1}{2}, 1\right]\end{array}\right.$, 
and the inversion of an edge as

$e^{-1}(t):=e(1-2 t)$

The holonomy (2.3) has two key properties

$h_{e_{1} \circ e_{2}}(A)=h_{e_{1}}(A) h_{e_{2}}(A), \quad h_{e^{-1}}(A)=h_{e}(A)^{-1}$.

The transformation behavior (2.2) of the connection $A$ under a gauge transformation leads to the corresponding transformation behavior of holonomy as

$h_{e}\left(A^{g}\right)=g(b(e)) h_{e}(A) g(f(e))^{-1}$,

where $b(e), f(e)$ denote the beginning and final points of $e$, respectively. The flux $\tilde{E}_{i}(S)$ of densitized triad $\tilde{E}_{i}^{a}$ through a 2 -surface $S$ is defined by

$\tilde{E}_{i}(S):=\int_{S} \epsilon_{a b c} \tilde{E}_{i}^{a}$,

where $\epsilon_{a b c}$ is the 3-dimensional Levi-Cività tensor density of weight -1 .

Consider a finite piecewise analytic graph $\gamma$ in $\Sigma$, which consists of analytic edges $e$ incident at vertices $v$. We insert a pseudo-vertex $\tilde{v}$ into each edge $e$ and split $e$ into two segments $s_{e}$ and $l_{e}$ such that $e=s_{e} \circ l_{e}^{-1}$ and the orientations of $s_{e}$ and $l_{e}$ are all outgoing from the two endpoints of $e$. We call the new graph the standard graph obtained from the original graph by splitting edges and adding pseudo-vertices. Denote the standard graph by $\gamma$, the set of its edges by $E(\gamma)$, and the set of vertices, containing the true vertices $v$ and pseudo-vertices $\tilde{v}$, by $V(\gamma)$. Our following discussion is based on the standard graphs.

To construct quantum kinematics, one has to extend the configuration space $\mathcal{A}$ of smooth connections to the space $\overline{\mathcal{A}}$ of distributional connections. A function $f$ on $\overline{\mathcal{A}}$ is said to be cylindrical with respect to a graph $\gamma$ if and only if it can be written as $f=f_{\gamma} \circ p_{\gamma}$, where $p_{\gamma}(A)=\left(h_{e_{1}}(A), \ldots, h_{e_{n}}(A)\right)$ and $e_{1}, \ldots, e_{n}$ are the edges of $\gamma$. Here $h_{e}(A)$ is the holonomy along $e$ evaluated at $A \in \overline{\mathcal{A}}$ and $f_{\gamma}$ is a complex-valued function on $S U(2)^{n}$. Since a function cylindrical with respect to a graph $\gamma$ is automatically cylindrical with respect to any graph bigger than $\gamma$, a cylindrical function is actually given by a whole equivalence class of functions $f_{\gamma}$. We will henceforth not distinguish the functions in one equivalence class. The set of cylindrical functions is denoted by $\operatorname{Cyl}(\overline{\mathcal{A}})$. The space $\operatorname{Cyl}(\overline{\mathcal{A}})$ can be completed as the kinematical Hilbert space $\mathcal{H}_{\text {kin }}:=L^{2}\left(\overline{\mathcal{A}}, \mathrm{d} \mu_{o}\right)$ with $\mathrm{d} \mu_{o}$ being the Ashtekar-Lewandowski measure.

Now let us consider the transformation behavior of the cylindrical function in order to understand the purpose of introducing the intertwiner. The cylindrical function can be decomposed by the representations $\pi_{j_{e}}\left(h_{e}(A)\right)$ of $h_{e}(A)$ as

$f_{\gamma}\left(\left\{h_{e}(A)\right\}_{e \in E(\gamma)}\right)=\bigoplus_{\vec{j} \equiv\left\{j_{e}\right\}_{e \in E(\gamma)}}\left(f_{\vec{j}} \cdot \bigotimes_{e \in E(\gamma)} \pi_{j_{e}}\left(h_{e}(A)\right)\right)$,

where $\cdot$ stands for contracting operator. Under finite gauge transformations, the above equation changes to

$$
\begin{aligned}
f_{\gamma}\left(\left\{h_{e}\left(A^{g}\right)\right\}_{e \in E(\gamma)}\right)= & \bigoplus_{\vec{j} \equiv\left\{j_{e}\right\}_{e \in E(\gamma)}}\left[f_{\vec{j}} \cdot \bigotimes_{e \in E(\gamma)}\left(\pi_{j_{e}}(g(v)) \cdot \pi_{j_{e}}\left(h_{e}(A)\right) \cdot \pi_{j_{e}}\left(g(\tilde{v})^{-1}\right)\right]\right. \\
& =\bigoplus_{\vec{j} \equiv\left\{j_{e}\right\}_{e \in E(\gamma)}}\left[f_{\vec{j}} \cdot \bigotimes_{v \in V(\gamma)}\left(\bigotimes_{b(e)=v} \pi_{j_{e}}(g(v))\right) \cdot \bigotimes_{e \in E(\gamma)} \pi_{j_{e}}\left(h_{e}(A)\right) \cdot \bigotimes_{\tilde{v} \in V(\gamma)}\left(\bigotimes_{f(e)=\tilde{v}} \pi_{j_{e}}\left(g(\tilde{v})^{-1}\right)\right)\right] \\
& =\bigoplus_{\vec{j} \equiv\left\{j_{e}\right\}_{e \in E(\gamma)}}\left[f_{\vec{j}} \cdot \bigotimes_{v \in V(\gamma)}\left(\bigoplus_{J}\left(i_{v}^{J}\right)^{-1} \cdot \pi_{J}(g(v)) \cdot i_{v}^{J}\right) \cdot \bigotimes_{e \in E(\gamma)} \pi_{j_{e}}\left(h_{e}(A)\right)\right.
\end{aligned}
$$




$$
\left.\cdot \bigotimes_{\tilde{v} \in V(\gamma)}\left(\bigoplus_{J^{\prime}}\left(i_{\tilde{v}}^{J^{\prime}}\right)^{*} \cdot \pi_{j_{e}}\left(g(\tilde{v})^{-1}\right) \cdot\left(\left(i_{\tilde{v}}^{J^{\prime}}\right)^{*}\right)^{-1}\right)\right]
$$

where in the last step we have employed the Clebsch-Gordan decomposition for the direct product of representations, $i_{v}^{J}$ is called the intertwining operator (tensor) in the representation theory of groups [39] and its components is the complex conjugate of (generalized) Clebsch-Gordan coefficients (CGCs) in quantum mechanics. Notice that $i_{v}^{J}$ and $\left(i_{\tilde{v}}^{J^{\prime}}\right)^{*}$ are independent for different vertices $v, \tilde{v} \in V(\gamma)$ and different total angular momenta $J$. Hence we can use them to expand the tensor $f_{\vec{j}}$ so that the cylindrical function in Eq. (2.10) can be written as

$$
f_{\gamma}\left(\left\{h_{e}(A)\right\}_{e \in E(\gamma)}\right)=\bigoplus_{\vec{j}=\left\{j_{e}\right\}_{e \in E(\gamma)}}\left\{\bigoplus_{J, J^{\prime}}\left[f_{\vec{j}}^{J, J^{\prime}} \cdot \bigotimes_{v \in V(\gamma)} i_{v}^{J} \cdot \bigotimes_{e \in E(\gamma)} \pi_{j_{e}}\left(h_{e}(A)\right) \cdot \bigotimes_{\tilde{v} \in V(\gamma)}\left(i_{\tilde{v}}^{J^{\prime}}\right)^{*}\right]\right\} .
$$

The orthogonality of CGCs ensures that the cylindrical function $f_{\gamma}\left(\left\{h_{e}(A)\right\}_{e \in E(\gamma)}\right)$ in $(2.12)$ is gauge invariant for $J=J^{\prime}=0$. Hence the tensor $i_{v}^{J}$ is also called the gauge-invariant (variant) intertwiner, associated to $v$, corresponding to $J$ takes 0 (nonvanishing value). The above discussion means that the basis of $\mathcal{H}_{\text {kin }}$ is ${ }^{2}$

$$
T_{\gamma, \vec{j}, \vec{i}}(A):=\bigotimes_{v \in V(\gamma)} i_{v} \cdot \bigotimes_{e \in E(\gamma)} \pi_{j_{e}}\left(h_{e}(A)\right) \cdot \bigotimes_{\tilde{v} \in V(\gamma)} i_{\tilde{v}}^{*},
$$

where $\cdot$ stands for contracting the upper (or former) indices of representation matrices $\pi_{j_{e}}\left(h_{e}(A)\right.$ ) with indices of intertwines $i_{v}$ at true vertices $v$, the lower (or later) indices of $\pi_{j_{e}}\left(h_{e}(A)\right)$ with indices of conjugate intertwiners $i_{\tilde{v}}^{*}$ at pseudo-vertices $\tilde{v}$, and we denote $\vec{i} \equiv\left\{i_{v}, i_{\tilde{v}}^{*}\right\}_{v, \tilde{v} \in V(\gamma)}$. The states (2.13) are called the spin-network states.

Given $n$ edges with $n$ spins $j_{1}, \ldots, j_{n}$ incident at a true $v$, the matrix elements of the intertwiner $i_{v}$ associated to $v$ read (see Appendix A for detailed explanation)

$$
\begin{aligned}
& \left(i_{v}^{J ; \vec{a}}\right)_{m_{1} m_{2} \cdots m_{n}}^{M} \equiv\left(\begin{array}{c}
i_{j_{1} \cdots j_{n}}^{J ; \vec{a}} \\
j_{m_{1} m_{2} \cdots m_{n}}
\end{array}\right) \quad M \\
& :=(-1)^{j_{1}-\sum_{i=2}^{n} j_{i}-J}\left\langle J M ; \vec{a} \mid j_{1} m_{1} j_{2} m_{2} \cdots j_{n} m_{n}\right\rangle \\
& =(-1)^{j_{1}-\sum_{i=2}^{n} j_{i}-J} \sum_{k_{2}, \ldots, k_{n-1}}\left\langle a_{2} k_{2} \mid j_{1} m_{1} j_{2} m_{2}\right\rangle \times\left\langle a_{3} k_{3} \mid a_{2} k_{2} j_{3} m_{3}\right\rangle \cdots\left\langle J M \mid a_{n-1} k_{n-1} j_{n} m_{n}\right\rangle,
\end{aligned}
$$

where $\left\langle J M ; \vec{a} \mid j_{1} m_{1} j_{2} m_{2} \cdots j_{n} m_{n}\right\rangle$ is the complex conjugate of generalized CGCs describing the coupling of $n$ angular momenta $j_{1}, \ldots, j_{n}$ to a total angular momentum $J$ in the standard coupling scheme (i.e., $j_{1}$ is first coupled to $j_{2}$ to give a resultant $a_{2}$, and then $a_{2}$ is coupled to $j_{3}$ to give $a_{3}$, and so on), and $\vec{a} \equiv\left\{a_{2}, \ldots, a_{n-1}\right\}$ denotes the set of the angular momenta appeared in the intermediate coupling. Notice that the intertwiner presented in Eq. (2.14), differing the factor $(-1)^{j_{1}-\sum_{i=2}^{n} j_{i}-J}$ from CGCs, is more convenient to be represented in graphical formula. The matrix elements of the conjugate intertwiner $i *$ associated to pseudo-vertex $\tilde{v}$ at which two incoming edges with the same spin $j$ meet are given by

$$
\left(i_{\tilde{v}}^{0^{*}}\right)^{n_{1} n_{2}} \equiv\left(i_{\tilde{v}}^{0^{*}}\right)_{0}^{n_{1} n_{2}}:=\left\langle j n_{1} j n_{2} \mid 00\right\rangle .
$$

The assignment of intertwiners to the true vertices and conjugate intertwiners to the pseudo-vertices is compatible with the transformation behavior (2.8) of holonomy. The CGCs are usually chosen to be real so that

$\left\langle j_{1} m_{1} j_{2} m_{2} \mid J M\right\rangle=\left\langle J M \mid j_{1} m_{1} j_{2} m_{2}\right\rangle$.

It is, therefore, not necessary to sedulously distinguish intertwiner from its conjugate when we do calculation. The gaugeinvariant spin-network states correspond to the states whose intertwiners in (2.14) associated to true vertices are specially

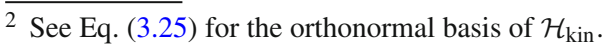


chosen such that the resulting angular momenta $J=0$. The normalized gauge-invariant/variant states consist of the orthonormal basis of the gauge-invariant/variant Hilbert space [40].

Two elementary operators in LQG are the holonomy and the flux operators. The holonomy operator acts as multiplication:

$\left[\hat{h}_{e_{I}}(A)\right]_{C}^{B} \cdot f_{\gamma}\left(h_{e_{1}}(A), \ldots, h_{e_{n}}(A)\right):=\left[\pi_{1 / 2}\left(h_{e_{I}}(A)\right)\right]_{C}^{B} f_{\gamma}\left(h_{e_{1}}(A), \ldots, h_{e_{n}}(A)\right)$.

Given a graph $\gamma$ and an oriented 2-surface $S$ with conormal $n_{a}^{S}$, the edges of $\gamma$ can be split into two halves at an interior point if necessary. Then one can get a graph $\gamma_{S}$ adapted to $S$ such that the edges of $\gamma_{S}$ belong to the following four types: (i) $e$ is up with respect to $S$ if $\dot{e}^{a}(0) n_{a}^{S}(e(0))>0$; (ii) $e$ is down with respect to $S$ if $\dot{e}^{a}(0) n_{a}^{S}(e(0))<0$; (iii) $e$ is inside with respect to $S$ if $e \cap S=e$; (iv) e is outside with respect to $S$ if $e \cap S=\emptyset$. Then the flux operator acts on a cylindrical function $f_{\gamma}$ with respect to the graph $\gamma$ adapted to $S$ as a derivative operator,

$$
\begin{aligned}
\hat{\tilde{E}}_{i}(S) \cdot f_{\gamma}\left(h_{e_{1}}(A), \ldots, h_{e_{n}}(A)\right) & :=-i \hbar\left\{f_{\gamma}\left(h_{e_{1}}(A), \ldots, h_{e_{n}}(A)\right), \tilde{E}_{i}(S)\right\} \\
& =\frac{\ell_{\mathrm{p}}^{2} \beta}{2} \sum_{e_{I} \in E(\gamma)} \varrho(e, S) J_{e_{I}}^{i} \cdot f_{\gamma}\left(h_{e_{1}}(A), \ldots, h_{e_{n}}(A)\right),
\end{aligned}
$$

where $\ell_{\mathrm{p}} \equiv \sqrt{\hbar \kappa}, \varrho(e, S)$ takes the values of $0,+1$ and -1 corresponding to whether the edge $e$ is inside/outside, up or down with respect to the surface $S$, and

$J_{e_{I}}^{i} \cdot f_{\gamma}\left(h_{e_{1}}(A), \ldots, h_{e_{I}}(A), \ldots, h_{e_{n}}(A)\right):=-\left.i \frac{\mathrm{d}}{\mathrm{d} t}\right|_{t=0} f_{\gamma}\left(h_{e_{1}}(A), \ldots, e^{t \tau_{i}} h_{e_{I}}(A), \ldots, h_{e_{n}}(A)\right)$

is the self-adjoint operator of the right-invariant vector field on the copy of $S U(2)$ corresponding to the $I$ th edge.

\section{Graphical method for LQG}

Graphic calculus has been introduced in LQG in a few papers (see e.g., $[13,14,18,21-23,28,29])$. Here we focus on the original Brink's graphical method and its suitable extension to LQG.

\subsection{Algebraic formula}

In LQG, under different physical considerations, one needs to construct operators, e.g., the geometric operators and the Hamiltonian operators, corresponding to their classical quantities based on the two elementary operators $\hat{h}_{e}(A)$ and $J_{e}^{i}$. The action of those operators on a given spin-network state will involve the actions of the two elementary operators. The action of $\hat{h}_{e}(A)$ on the spin-network states involves essentially the decomposition of the tensor product representation of $S U(2)$, which is well known as the Clebsch-Gordan series

$\left[\pi_{j_{1}}(g)\right]_{n_{1}}^{m_{1}}\left[\pi_{j_{2}}(g)\right]^{m_{2}}{ }_{n_{2}}=\sum_{J, M, N}\left(\left(i_{j_{1} j_{2}}^{J}\right)^{-1}\right)_{M}{ }^{m_{1} m_{2}} \times\left[\pi_{J}(g)\right]^{M}{ }_{N}\left(i_{j_{1} j_{2}}^{J}\right)_{n_{1} n_{2}}{ }^{N}$,

where $\left(i_{j_{1} j_{2}}^{J}\right)_{n_{1} n_{2}}{ }^{N} \equiv(-1)^{j_{1}-j_{2}-J}\left\langle J N \mid j_{1} n_{1} j_{2} n_{2}\right\rangle$. The fact that the operator $i_{j_{1} j_{2}}^{J}$ is unitary and its matrix elements take real numbers results in

$\left(\left(i_{j_{1} j_{2}}^{J}\right)^{-1}\right)_{M}^{m_{1} m_{2}}=\left(i_{j_{1} j_{2}}^{J}\right)_{m_{1} m_{2}}^{M}$.

For a spin-network state $T_{\gamma, \vec{j}, \vec{i}}(A)$ on a graph $\gamma$, we consider a true vertex $v \in V(\gamma)$ at which $n$ edges $e_{1}, \ldots e_{n}$ incident and denote $T_{\gamma, \vec{j}, \vec{i}}^{v}(A)$ the terms, in $T_{\gamma, \vec{j}, \vec{i}}(A)$, directly associated to $v$. Then the action of the holonomy operator $\left[\hat{h}_{e_{I}}(A)\right]^{B}{ }_{C}$ on $T_{\gamma, \vec{j}, \vec{i}}^{v}(A)$ reads 


$$
\begin{aligned}
& {\left[\hat{h}_{e_{I}}(A)\right]^{B}{ }_{C} \cdot T_{\gamma, \vec{j}, \vec{i}}^{v}(A)=\left(i_{v}^{J ; \vec{a}}\right)_{m_{1} \cdots m_{I} \cdots m_{n}}\left[\pi_{j_{1}}\left(h_{e_{1}}\right)\right]^{m_{1}}{ }_{n_{1}} \cdots\left[\pi_{j_{I}}\left(h_{e_{I}}\right)\right]_{n_{I}}^{m_{I}}\left[\pi_{1 / 2}\left(h_{e_{I}}\right)\right]_{C}^{B} \cdots\left[\pi_{j_{n}}\left(h_{e_{n}}\right)\right]^{m_{n}} n_{n}} \\
& =\left(i_{v}^{J ; \vec{a}}\right)_{m_{1} \cdots m_{I} \cdots m_{n}}^{M}\left[\pi_{j_{1}}\left(h_{e_{1}}\right)\right]_{n_{1}}^{m_{1}} \cdots \sum_{j_{I}^{\prime}, m_{I}^{\prime}, n_{I}^{\prime}}\left(\left(i_{j_{I} 1 / 2}^{j_{I}^{\prime}}\right)^{-1}\right)_{m_{I}^{\prime}}^{m_{I} B}\left[\pi_{j_{I}^{\prime}}\left(h_{e_{I}}\right)\right]_{n_{I}^{\prime}}^{m_{I}^{\prime}}\left(i_{j_{I} 1 / 2}^{j_{I}^{\prime}}\right)_{n_{I} C}{ }_{n_{I}^{\prime}} \cdots\left[\pi_{j_{n}}\left(h_{e_{n}}\right)\right]^{m_{n}} n_{n} .
\end{aligned}
$$

where $\left[\pi_{j_{I}}\left(h_{e_{I}}\right)\right]^{m_{I}}{ }_{n_{I}} \equiv\left[\pi_{j_{I}}\left(h_{e_{I}}(A)\right)\right]_{{ }_{n}}^{m_{I}}$. On the other hand, the action of $J_{e_{I}}^{i}$ defined in Eq. (2.19) on $T_{\gamma, \vec{j}, \vec{i}}^{v}(A)$ reads

$$
\begin{aligned}
J_{e_{I}}^{i} \cdot T_{\gamma, \vec{j}, \vec{i}}^{v}(A) & =\left(i_{v}^{J ; \vec{a}}\right)_{m_{1} \cdots m_{I} \cdots m_{n}}{ }^{M}\left[\pi_{j_{1}}\left(h_{e_{1}}\right)\right]^{m_{1}}{ }_{n_{1}} \cdots\left(-\left.i \frac{\mathrm{d}}{\mathrm{d} t}\right|_{t=0}\left[\pi_{j_{I}}\left(e^{t \tau_{i}} h_{e_{I}}\right)\right]^{m_{I}}{ }_{n_{I}}\right) \cdots\left[\pi_{j_{n}}\left(h_{e_{n}}\right)\right]^{m_{n}}{ }_{n_{n}} \\
& =\left(i_{v}^{J ; \vec{a}}\right)_{m_{1} \cdots m_{I} \cdots m_{n}}{ }^{M}\left[\pi_{j_{1}}\left(h_{e_{1}}\right)\right]^{m_{1}}{ }_{n_{1}} \cdots\left(-i\left[\pi_{j_{I}}\left(\tau_{i}\right)\right]^{m_{I}}{ }_{m_{I}^{\prime}}\left[\pi_{j_{I}}\left(h_{e_{I}}\right)\right]^{m_{I}^{\prime}}{ }_{n_{I}}\right) \cdots\left[\pi_{j_{n}}\left(h_{e_{n}}\right)\right]^{m_{n}}{ }_{n_{n}} \\
& =\left[\left(i_{v}^{J ; \vec{a}}\right)_{m_{1} \cdots m_{I}^{\prime} \cdots m_{n}}{ }^{M}\left(-i\left[\pi_{j_{I}}\left(\tau_{i}\right)\right]^{m_{I}^{\prime}}{ }_{m_{I}}\right)\right]\left[\pi_{j_{1}}\left(h_{e_{1}}\right)\right]^{m_{1}}{ }_{{ }_{n}} \cdots\left[\pi_{j_{I}}\left(h_{e_{I}}\right)\right]^{m_{I}} \cdots\left[\pi_{n_{I}}\left(h_{e_{n}}\right)\right]^{m_{n}}{ }_{n_{n}},
\end{aligned}
$$

which indicates that $J_{e_{I}}^{i}$ leaves $\gamma$ and $\vec{j}$ invariant, but does change the intertwiner associated to $v$ by contracting matrix elements of the $i$ th $\tau$ with the intertwiner in the following way:

$$
J_{e_{I}}^{i} \cdot\left(i_{v}^{J ; \vec{a}}\right)_{m_{1} \cdots m_{I} \cdots m_{n}}^{M}=\left(i_{v}^{J ; \vec{a}}\right)_{m_{1} \cdots m_{I}^{\prime} \cdots m_{n}}^{M}\left(-i\left[\pi_{j_{I}}\left(\tau_{i}\right)\right]_{m_{I}}^{m_{I}^{\prime}}\right) .
$$

However, in practical calculation, it is not convenient to directly compute the contraction of matrix elements of $\tau_{i}$ with an intertwiner. One usually introduces the irreducible tensor operators [41], or the spherical tensors of $\tau_{i}$, to replace the original $\tau_{i}$ for a reason that will become clear in a moment. The spherical tensors $\tau_{\mu}(\mu=0, \pm 1)$, corresponding to $\tau_{i}(i=1,2,3)$, are defined by

$\tau_{0}:=\tau_{3}, \quad \tau_{ \pm 1}:=\mp \frac{1}{\sqrt{2}}\left(\tau_{1} \pm i \tau_{2}\right)$.

Then the contraction of matrix elements of $\tau_{i}$ with an intertwiner is transformed to that of their tensor operators with the intertwiner. The matrix elements $\left[\pi_{j}\left(\tau_{\mu}\right)\right]^{m^{\prime}}{ }_{m}$ can be related to the $3 j$-symbols (or CGCs) by (see Appendix B.1 for a proof)

$\left[\pi_{j}\left(\tau_{\mu}\right)\right]_{m}^{m^{\prime}}=\frac{i}{2} \sqrt{2 j(2 j+1)(2 j+2)}\left(\begin{array}{ccc}1 & j & j \\ \mu & m^{\prime \prime} & m\end{array}\right) C_{(j)}^{m^{\prime \prime} m^{\prime}}$,

where $C_{(j)}^{m^{\prime \prime} m^{\prime}}:=(-1)^{j+m^{\prime}} \delta_{m^{\prime},-m^{\prime \prime}}$ is the contravariant "metric" tensor on the irreducible representation space $\mathcal{H}_{j}$ of $S U(2)$ with spin $j$ (see Appendix A.1 for a detailed explaination for the $C_{(j)}^{m^{\prime \prime} m^{\prime}}$ ) [42]. The spherical tensor $\tau_{\mu}$ generates the self-adjoint right-invariant operator $J_{e_{I}}^{\mu}$ defined by

$J_{e_{I}}^{\mu} \cdot f_{\gamma}\left(h_{e_{1}}(A), \ldots, h_{e_{I}}(A), \ldots, h_{e_{n}}(A)\right):=-\left.i \frac{\mathrm{d}}{\mathrm{d} t}\right|_{t=0} f_{\gamma}\left(h_{e_{1}}(A), \ldots, e^{t \tau_{\mu}} h_{e_{I}}(A), \ldots, h_{e_{n}}(A)\right)$.

The action of $J_{e_{I}}^{\mu}$ on $\left(i_{v}^{J ; \vec{a}}\right)_{m_{1} \cdots m_{I} \cdots m_{n}}{ }^{M}$ reads

$J_{e_{I}}^{\mu} \cdot\left(i_{v}^{J ; \vec{a}}\right)_{m_{1} \cdots m_{I} \cdots m_{n}}^{M}=\left(i_{v}^{J ; \vec{a}}\right)_{m_{1} \cdots m_{I}^{\prime} \cdots m_{n}}{ }^{M}\left(-i\left[\pi_{j_{I}}\left(\tau_{\mu}\right)\right]_{m_{I}}^{m_{I}^{\prime}}\right)$.

Any gauge-invariant operator, e.g., the volume operator considered in this paper, defined by $J^{i}$ s can be expressed in terms of the corresponding $J^{\mu}$ s. Hence its action on the spin-network states is essentially equivalent to contracting $3 j$-symbols (or CGCs) with corresponding intertwiners. 


\subsection{Graphical representation and graphical calculation}

The basic components of the original Brink's graphical representation and the simple rules of transforming graphs are presented in Appendix A.1. In graphical representation, the $3 j$-symbol is represented by an oriented node with three lines, each of which represents a value of $j$, i.e.,

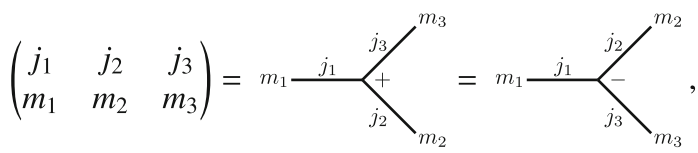

where - and + denote the clockwise orientation and the anti-clockwise orientation, respectively. A rotation of the diagram does not change the cyclic order of lines, and the angles between two lines as well as their lengths at a node have no significance. The "metric" tensor $C_{m^{\prime} m}^{(j)}$ in Eq. (A.6) which occurs in the contraction of two $3 j$-symbols with the same values of $j$ is denoted by a line with an arrow on it, i.e.,

$C_{m^{\prime} m}^{(j)}=(-1)^{j-m} \delta_{m,-m^{\prime}}=(-1)^{j+m^{\prime}} \delta_{m,-m^{\prime}}={ }_{m} \longrightarrow{ }^{\prime}$,

and its inverse in Eq. (A.7) can be expressed as

$C_{(j)}^{m m^{\prime}}=(-1)^{j-m} \delta_{m,-m^{\prime}}=(-1)^{j+m^{\prime}} \delta_{m,-m^{\prime}}={ }_{m} \longrightarrow m^{\prime}$.

Summation over the magnetic quantum numbers $m$ is graphically represented by joining the free ends of the corresponding lines. The contraction of a $3 j$-symbol with a "metric" is represented by a node with one arrow, which provides a way to represent the CGC, e.g.,

$$
\begin{aligned}
\left\langle j_{3} m_{3} \mid j_{1} m_{1} j_{2} m_{2}\right\rangle & =(-1)^{j_{1}-j_{2}-j_{3}} \sqrt{2 j_{3}+1}\left(\begin{array}{ccc}
j_{1} & j_{2} & j_{3} \\
m_{1} & m_{2} & m_{3}^{\prime}
\end{array}\right) C_{\left(j_{3}\right)}^{m_{3}^{\prime} m_{3}} \\
& =(-1)^{j_{1}-j_{2}-j_{3}}\left(i_{j_{1} j_{2}}^{j_{3}}\right)_{m_{1} m_{2}} m_{3}=(-1)^{j_{1}-j_{2}-j_{3}} \sqrt{2 j_{3}+1} \underbrace{m_{j_{2}}^{j_{1}}}_{m_{2}} m_{j_{3}}^{j_{3}} .
\end{aligned}
$$

To give a precise way of presenting the CGC as Eq. (3.13) is the main motivation for Brink to modify the original Yutsis scheme [30,31]. Hence the intertwiner $\left(i_{v}^{J ; \vec{a}}\right)_{m_{1} m_{2} \cdots m_{n}}{ }^{M}$ in Eq. (2.14) associated to a true vertex $v$, from which $n$ edges are outgoing, is represented in a graphical formula by Eq. (A.38) as (see Appendix A.2 for a detailed interpretation)

$\left(i_{v}^{J ; \vec{a}}\right)_{m_{1} m_{2} \cdots m_{n}}{ }^{M} \equiv\left(i_{j_{1} \cdots j_{n}}^{J ; \vec{a}}\right)_{m_{1} m_{2} \cdots m_{n}}{ }^{M}=\prod_{i=2}^{n-1} \sqrt{2 a_{i}+1} \sqrt{2 J+1} \underbrace{m_{a_{2}}}_{j_{1}} \underbrace{\left.m_{a_{n-1}}\right|_{J} ^{m_{2}}}_{j_{2}} M$.

Now we will extend Brink's representation and propose a graphical representation for the unitary irreducible representation $\pi_{j}$ of $S U(2)$. The matrix element $\left[\pi_{j}(g)\right]^{m}{ }_{n}$ is denoted by a blue line with a hollow arrow (triangle) in it as

$\left[\pi_{j}(g)\right]_{n}^{m}=: m \longrightarrow{ }^{j}=n$.

The orientation of the arrow is from its row index $m$ to its column index $n$. For group elements such as the holonomies $h_{e} \equiv h_{e}(A)$ of the connection $A$ along an edge $e$, their matrix elements can simply be represented by

$\left[\pi_{j}\left(h_{e}\right)\right]^{m}{ }_{n}=m \longrightarrow \overbrace{j}^{j} n$.

The advantages of the above graphical representation are the following: (i) The edge and the irreducible representation of the holonomy along the edge have been represented by the elements $e$ and $j$ labeling the line; (ii) the orientation of $e$ with respect to the vertices has been reflected by the orientation of the arrow on the line; (iii) the row index (the tensor index of $\mathcal{H}_{j}^{*}$ ) and the column index (the tensor index of $\mathcal{H}_{j}$ ) have been represented by the two indices $m$ and $n$, respectively, labeling the 
starting and the ending points of the line; (iv) the matrix element $\left[\pi_{j}\left(h_{e}\right)\right]^{m}{ }_{n}$ and the "metric" tensor $C_{m^{\prime} m}^{(j)}$ in the graphical formula are distinguished by different colors (blue v.s. black) and elements (two v.s. one) of the lines; (v) the coupling rules of the representations of holonomies match Brink's representations for the CGC (see Eq. (3.18)). By Eqs. (2.7) and (A.57), the matrix elements of the inverse of a holonomy can be represented by

$\left[\pi_{j}\left(h_{e}^{-1}\right)\right]_{m}^{n}=\left[\pi_{j}\left(h_{e^{-1}}\right)\right]_{m}^{n}=C_{m m^{\prime}}^{(j)}\left[\pi_{j}\left(h_{e}\right)\right]^{m^{\prime}}{ }_{n^{\prime}} C_{(j)}^{n^{\prime} n}=n \underset{j}{e^{-1}} m=n \longrightarrow \underbrace{e}_{j} \longrightarrow m$.

The Clebsch-Gordan series in (3.1) yield the coupling rules of representations of holonomies as ${ }^{3}$

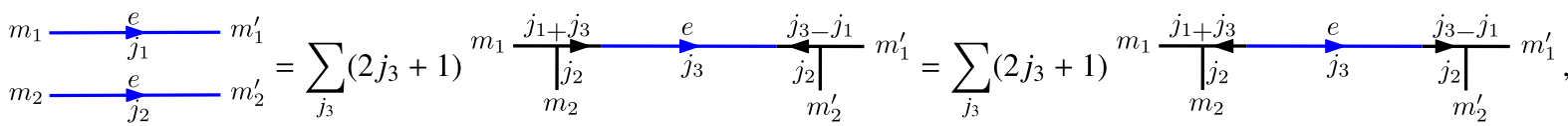

and

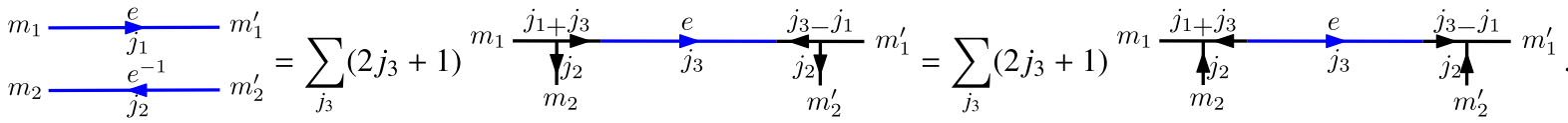

The action of $\left[\hat{h}_{e_{I}}(A)\right]^{B}{ }_{C}$ on the spin-network state $T_{\gamma, \vec{j}, \vec{i}}^{v}(A)=\left(i_{v}^{J ; \vec{a}}\right)_{m_{1} \cdots m_{I} \cdots m_{n}}{ }^{M}\left[\pi_{j_{1}}\left(h_{e_{1}}\right)\right]^{m_{1}}{ }_{n_{1}} \cdots\left[\pi_{j_{I}}\left(h_{e_{I}}\right)\right]^{m_{I}}{ }_{n_{I}} \cdots$ $\left[\pi_{j_{n}}\left(h_{e_{n}}\right)\right]^{m_{n}}{ }_{n_{n}}$ can be represented by
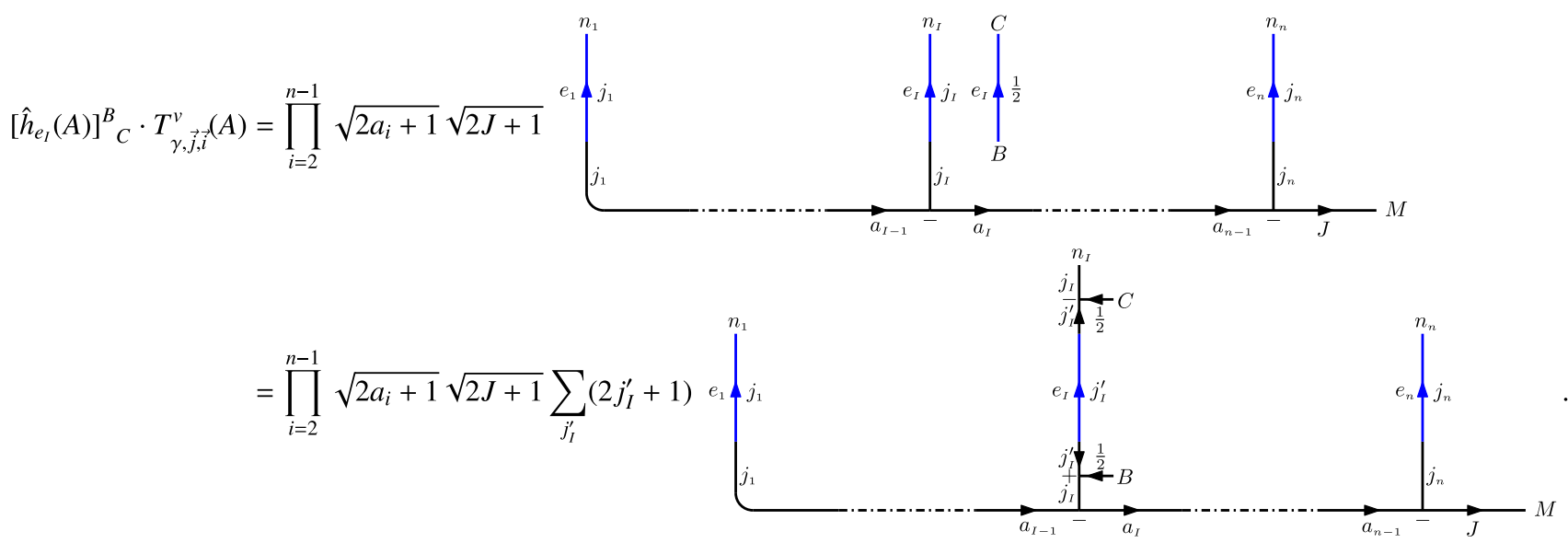

The spherical tensor $\left[\pi_{j}\left(\tau_{\mu}\right)\right]^{m^{\prime}}{ }_{m}$ in Eq. (3.7) can be represented graphically by

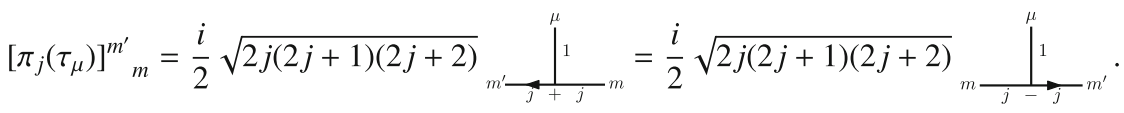

Hence the action of $J_{e_{I}}^{\mu}$ on $\left(i_{v}{ }_{v} ; \vec{a}\right)_{m_{1} m_{2} \cdots m_{n}}{ }^{M}$ in (3.9) can be represented by

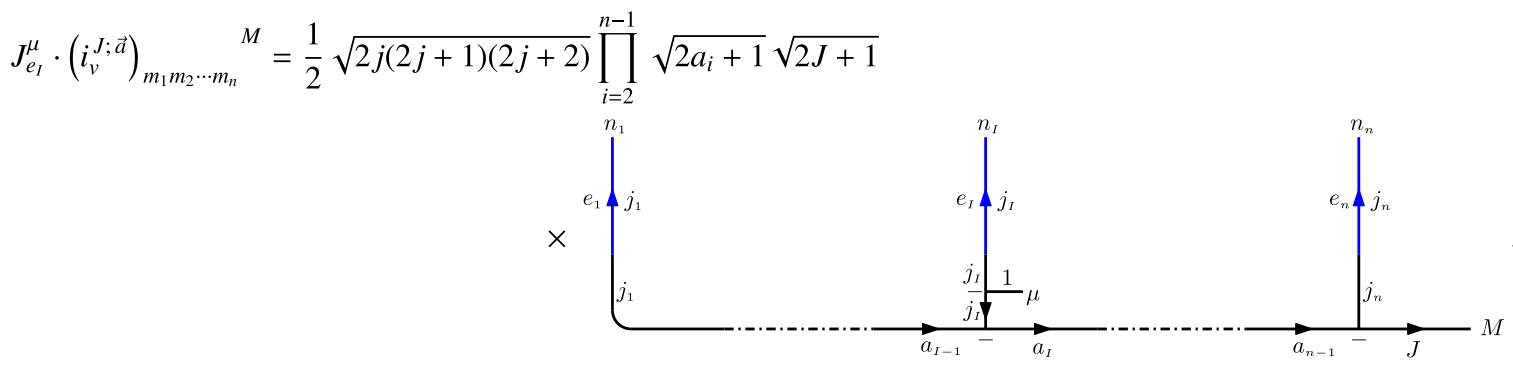

${ }^{3}$ Similar calculus based on the Varshalovich method were used in LQG (see e.g. [14]). 
Up to now, based on the Brink original graphical representation and its suitable extension to the irreducible representation of holonomy, the two elementary operators in LQG, the holonomy operator and the flux operator (essentially the self-adjoint right-invariant operator), and the spin-network states of the kinematical Hilbert space have been uniquely represented by corresponding graphs. Hence, in the graphical method, the actions of any well-defined operators in the kinematical space, for instances, the volume operator, the Hamiltonian operator and the inverse operator considered in this paper, on a spin-network state can be derived by the simple rules of transforming graphs (see Appendix A.2).

The starting point of our scheme is the so-called standard graph $\gamma_{\text {std }}$, which is obtained from its original graph $\gamma_{\text {org }}$ by splitting edges and adding pseudo-vertices. We still need to show that the spin-network function associated to the original graph $\gamma_{\text {org }}$ is equivalent to the one associated to its corresponding standard graph $\gamma_{\text {std }}$ acting by an operator. Recall that the standard graph $\gamma_{\text {std }}$ is obtained from $\gamma_{\text {org }}$ by the following procedure. We insert a pseudo-vertex $\tilde{v}$ into each edge $e$ of $\gamma_{\text {org }}$ and split $e$ into two segments $s_{e}$ and $l_{e}$, such that $e=s_{e} \circ l_{e}^{-1}$ and the orientations of $s_{e}$ and $l_{e}$ are all outgoing from the two endpoints of $e$. The standard graph $\gamma_{\text {std }}$ consists of the new segments $s_{e}$ and $l_{e}$, the new adding pseudo-vertices $\tilde{v}$, and the (old) vertices of $\gamma_{\text {org }}$. We can transform the spin networks based on the original graph into those on its standard graph by explicit

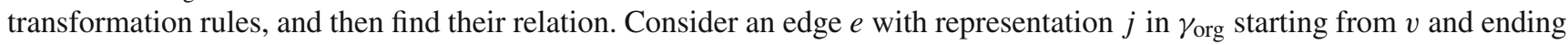
at $v^{\prime}$, assigning the intertwiners $i_{v}$ and $i_{v^{\prime}}$, respectively. We assume that the edge $e$ in the original graph $\gamma_{\text {org }}$ is regarded as the $k$ th edge and the $k^{\prime}$ th edge in the set of edges which incident at $v$ and $v^{\prime}$, respectively, i.e., $b(e)=b\left(e_{k}\right), f(e)=f\left(e_{k^{\prime}}^{\prime}\right)$. The relevant ingredient of a spin-network state associated to the edge $e$ takes the form [see (A.39) for the graphical representation of the intertwiner associated to $v$ at which there are coming and outgoing edges, (3.16) and (3.17) for the graphical representation of the holonomy]

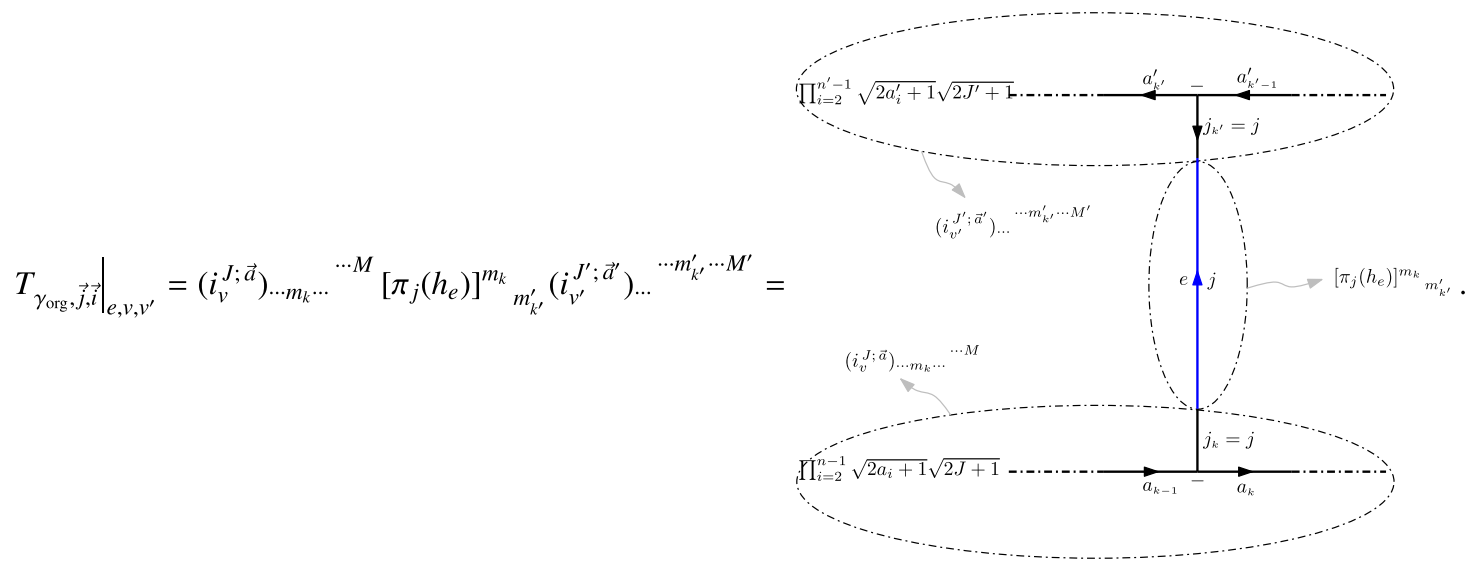

The transformation from $\gamma_{\text {org }}$ to $\gamma_{\text {std }}$ induces the following transformation in the spin-network state:
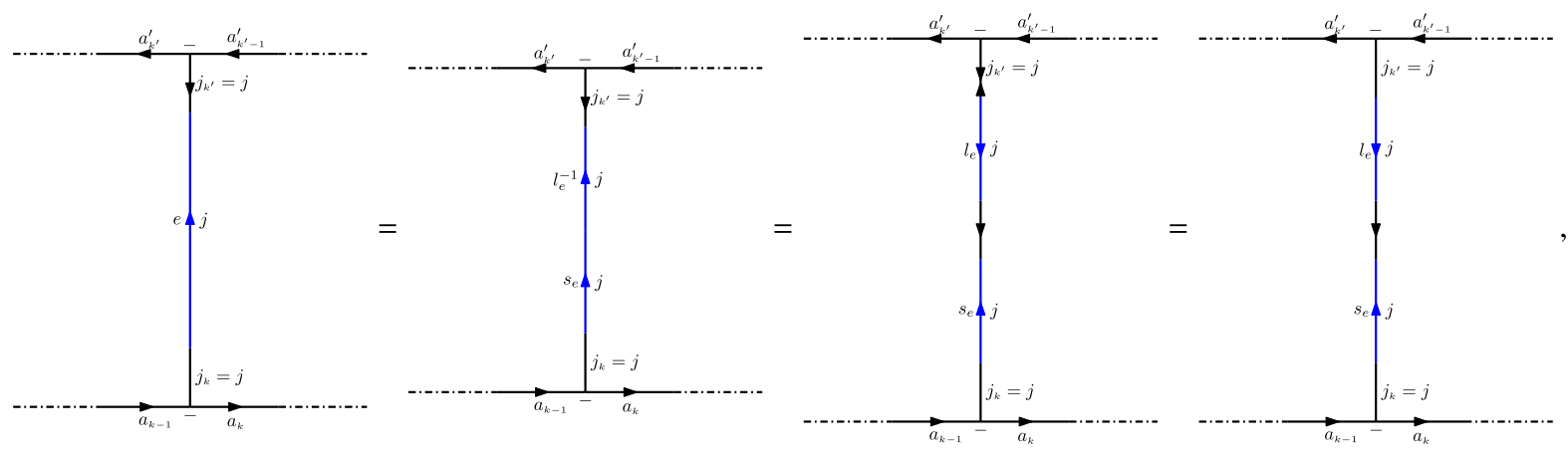

where we have used (3.17) in the second step and the rule (A.41) to remove two arrows in the last step. Repeating the above procedure, we can transform the spin-network states associated to the origin graph into those corresponding to its standard 
graph. By this trick, we finally find out the corresponding relation of the spin-network states between the original graph $\gamma_{\text {org }}$ and its standard graph $\gamma_{\text {std }}$. The intertwiners associated to the vertices of the origin graph is replaced by its standard formula, and adding the pseudo-vertex $\tilde{v}$ for each edge $e$ with a divalent intertwiner is graphically represented by an arrow with an orientation opposed to that of the original edge. ${ }^{4}$

We now show by the graphical method that the spin-network states can become orthonormal to each other. The normalized spin-network state takes the form

$$
T_{\gamma, \vec{j}, \vec{i}}^{\mathrm{norm}}(A):=\bigotimes_{v \in V(\gamma)} i_{v} \cdot \bigotimes_{e \in E(\gamma)} \sqrt{2 j_{e}+1} \pi_{j_{e}}\left(h_{e}(A)\right) \cdot \bigotimes_{\tilde{v} \in V(\gamma)} i_{\tilde{v}}^{*}
$$

The scalar product of the spin-network states is defined by

$$
\left(T_{\gamma^{\prime}, \vec{j}^{\prime}, \vec{i}^{\prime}}^{\mathrm{norm}}, T_{\gamma, \vec{j}, \vec{i}}^{\mathrm{norm}}\right)_{\mathcal{H}_{\mathrm{kin}}}:=\int_{S U(2)^{|E(\tilde{\gamma})|}} \prod_{e \in E(\tilde{\gamma})} \mathrm{d} \mu_{H}\left(h_{e}\right) \overline{T_{\gamma^{\prime}, \vec{j}^{\prime}, \vec{i}^{\prime}}^{\mathrm{norm}}(A)} T_{\gamma, \vec{j}, \vec{i}}^{\mathrm{norm}}(A),
$$

where $\tilde{\gamma}$ is any graph bigger than $\gamma$ and $\gamma^{\prime},|E(\tilde{\gamma})|$ denotes the number of the edges in $\tilde{\gamma}$, and $\mathrm{d} \mu_{H}(g)$ is the Haar measure on $S U$ (2). If $\gamma$ differs from $\gamma^{\prime}$, e.g., there is an edge $e^{\prime}$ with spin $j_{e^{\prime}}^{\prime}$ belonging to $\gamma^{\prime}$ but not $\gamma$, then the orthogonality relation,

$$
\int_{S U(2)} \mathrm{d} \mu_{H}(g) \overline{\left[\pi_{j^{\prime}}(g)\right]_{n^{\prime}}^{m^{\prime}}}\left[\pi_{j}(g)\right]_{n}^{m}=\frac{\delta_{j, j^{\prime}}}{2 j+1} \delta^{m, m^{\prime}} \delta_{n, n^{\prime}}
$$

implies that the corresponding integration in (3.26) becomes

$$
\int_{S U(2)} \mathrm{d} \mu_{H}\left(h_{e^{\prime}}\right)\left[\pi_{j_{e^{\prime}}^{\prime}}\left(h_{e^{\prime}}\right)\right]_{n^{\prime}}^{m^{\prime}}=0
$$

Hence the non-trivial result corresponds to the case $\gamma=\gamma^{\prime}$. Thus (3.26) reduces to

$$
\begin{aligned}
\left(T_{\gamma^{\prime}, \vec{j}^{\prime}, \vec{i}^{\prime}}^{\mathrm{norm}}, T_{\gamma, \vec{j}, \vec{i}}^{\mathrm{norm}}\right)_{\mathcal{H}_{\mathrm{kin}}} & =\delta_{\gamma, \gamma^{\prime}} \int_{S U(2)^{|E(\tilde{\gamma})|}} \prod_{e \in E(\tilde{\gamma})} \mathrm{d} \mu_{H}\left(h_{e}\right) \overline{T_{\gamma, \vec{j}^{\prime}, \vec{i}^{\prime}}^{\text {norm }}(A)} T_{\gamma, \vec{j}, \vec{i}}^{\mathrm{norm}}(A) \\
& =\delta_{\gamma, \gamma^{\prime}} \int_{S U(2)^{|E(\gamma)|}} \prod_{e \in E(\gamma)} \mathrm{d} \mu_{H}\left(h_{e}\right) \overline{T_{\gamma, \overrightarrow{j^{\prime}, \vec{i}^{\prime}}}^{\text {norm }}(A)} T_{\gamma, \vec{j}, \vec{i}}^{\text {norm }}(A),
\end{aligned}
$$

where in the second step we have used the fact that the Haar measure is normalized. By integrating over all representation functions on the edges, one can obtain the contract of the complex conjugation of intertwiners with the corresponding intertwiners at vertices. Thus we have

\footnotetext{
$\overline{4}$ The intertwiner, a line with an arrow, associated to the pseudo-vertex $\tilde{v}$, is not normalizable since we adopt $\pi_{j_{e}}\left(h_{e}(A)\right)$ rather that its normalized form $\sqrt{2 j_{e}+1} \pi_{j_{e}}\left(h_{e}(A)\right)$ in the spin-network function (see Eq. (2.13)). If the original spin network is normalized, the intertwiner associated to $\tilde{v}$ will automatically be normalized. Then it will be expressed as $\frac{1}{\sqrt{2 j+1}}$ times a line with a spin- $j$ arrow in the graphical representation in Eq. (3.24).
} 


$$
\begin{aligned}
\left(T_{\gamma^{\prime}, \vec{j}^{\prime}, \vec{i}^{\prime}}^{\text {norm }}, T_{\gamma, \vec{j}, \vec{i}}^{\text {norm }}\right)_{\mathcal{H}_{\text {kin }}} & =\delta_{\gamma, \gamma^{\prime}} \prod_{e \in E(\gamma)} \delta_{j_{e}, j_{e}^{\prime}} \prod_{v \in V(\gamma)} \sum_{m_{1}, \cdots, m_{n}} \overline{\left(i_{v}^{J^{\prime} ; \vec{a}^{\prime}}\right)_{m_{1} \cdots m_{n}}{ }^{M^{\prime}}}\left(i_{v}^{J ; \vec{a}}\right)_{m_{1} \cdots m_{n}}{ }^{M} \\
& =\delta_{\gamma, \gamma^{\prime}} \prod_{e \in E(\gamma)} \delta_{j_{e}, j_{e}^{\prime}} \prod_{v \in V(\gamma)} \sum_{m_{1}, \cdots, m_{n}}\left(i_{v}^{J^{\prime} ; \vec{a}^{\prime}}\right)_{m_{1} \cdots m_{n}}{ }^{M^{\prime}}\left(i_{v}^{J ; \vec{a}}\right)_{m_{1} \cdots m_{n}}{ }^{M} \\
& =\delta_{\gamma, \gamma^{\prime}} \prod_{e \in E(\gamma)} \delta_{j_{e}, j_{e}^{\prime}} \prod_{v \in V(\gamma)} \prod_{i=2}^{n-1} \sqrt{\left(2 a_{i}+1\right)\left(2 a_{i}^{\prime}+1\right)} \sqrt{(2 J+1)\left(2 J^{\prime}+1\right)} \\
& =\delta_{\gamma, \gamma^{\prime}} \prod_{e \in E(\gamma)} \delta_{j_{e}, j_{e}^{\prime}} \prod_{v \in V(\gamma)} \prod_{i=2}^{n-1} \delta_{a_{i}, a_{i}^{\prime}} \delta_{J, J^{\prime}} \delta_{M, M^{\prime}} \\
& \equiv \delta_{\gamma, \gamma^{\prime}} \prod_{e \in E(\gamma)} \delta_{j_{e}, j_{e}^{\prime}} \prod_{v \in V(\gamma)} \delta_{i_{v}, i_{v}^{\prime}} \\
& \equiv \delta_{\gamma, \gamma^{\prime}} \delta_{\vec{j}, \overrightarrow{j^{\prime}}} \delta_{\vec{i}, \vec{i}^{\prime}},
\end{aligned}
$$

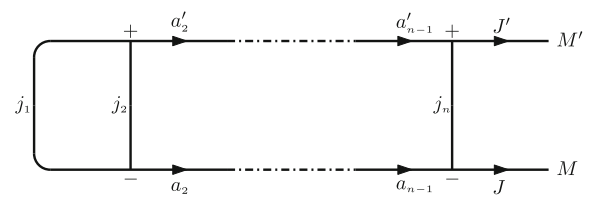

where in the second step we have used the fact that the CGs (and thus the GCGs) are real, in the fourth step we have used Eqs. (A.45) and (A.41). Note that the result of Eq. (3.30) is based on the premise that the intertwiners at the same vertex $v$ involve the same coupling scheme. If different coupling schemes at the same vertex were chosen, certain additional multiplication of $6 j$-symbols would appear in the result. If the spin-network functions are gauge invariant, corresponding to $J=0$ and $M=0$, the two Kronecker delta functions $\delta_{J, J^{\prime}}$ and $\delta_{M, M^{\prime}}$ will not appear in Eq. (3.30).

\section{The volume operator}

One of the important achievements in LQG is that the theory itself predicts that some geometric operators, such as area operator and volume operator, have discretized spectra. Some volume operator was also constructed for spin-foam models $[43,44]$. There are two versions of volume operator in canonical LQG. We only consider the volume operator defined in $[10,11]$, which passed the consistency check in the quantum kinematical framework and was used to define a Hamiltonian constraint operator in LQG $[16,45,46]$. In this section, we will briefly review the construction of the volume operator. Then the graphical method, introduced in Sect. 3, will be used to derive the matrix element of the volume operator.

4.1 A brief review of the construction of the volume operator

Classically, the volume function for a given open region $R$ reads

$V(R):=\int_{R} \mathrm{~d}^{3} x \sqrt{|\operatorname{det}(q)|}=\int_{R} \mathrm{~d}^{3} x \sqrt{\left|\frac{1}{3 !} \epsilon^{i j k}{\underset{\sim}{\epsilon b c}}_{a b} \tilde{E}_{i}^{a} \tilde{E}_{j}^{b} \tilde{E}_{k}^{c}\right|}$,

where $\operatorname{det}(q)$ denotes the determinant of the 3-metric $q_{a b}$. To quantize the volume function, a suitable regularization procedure is needed which involves smearing $\tilde{E}_{i}^{a}$. We now introduce the regularization adopted by Ashtekar and Lewandows in [10].

For given $R \in \Sigma$, we fix a global coordinates $\left\{x^{a}, a=1,2,3\right\}$ in a neighborhood of $R$ in $\Sigma$ and partition $\mathcal{P}_{\epsilon}$ of $R$ into a family $\mathscr{C}$ of closed cubes $C$ with coordinate volume $\epsilon^{3}$. For each $C$, one arranges three 2 -surface $S^{1}, S^{2}, S^{3}$, defined by $x^{a}=$ const $^{a}$, intersecting in the interior of $C$. One smears these three densitized triads on those three 2 -surfaces for each cell $C$ in a given partition to give a regularized version of (4.1) as [10]

$V_{\mathrm{AL}}^{\mathcal{P}_{\epsilon}}(R):=\sum_{C \in \mathscr{C}} \sqrt{\left|\frac{1}{3 !} \epsilon^{i j k} \epsilon_{a b c} \tilde{E}_{i}\left(S^{a}\right) \tilde{E}_{j}\left(S^{b}\right) \tilde{E}_{k}\left(S^{c}\right)\right|}$.

It is easy to see that (4.2) reduces to (4.1) as $\epsilon \rightarrow 0$. The above regularization procedure is called internal because triads are smeared over three surfaces passing the interior of the cell. It is straightforward to promote the regularized formula (4.2) to 
its quantum operator by replacing the fluxes by their operators. It is convenient to introduce the permissible partitions $\mathcal{P}_{\epsilon}^{\gamma}$ (for sufficiently small $\epsilon$ ) adapted to a given standard graph $\gamma$ (see [10] for details). One then obtains the regulated operator

$$
\hat{V}_{\mathrm{AL}}^{\mathcal{P}_{\epsilon}^{\gamma}}(R) \cdot f_{\gamma}:=\ell_{\mathrm{p}}^{3} \beta^{\frac{3}{2}} \sum_{v \in V(\gamma) \cap R} \sqrt{\left|\frac{1}{3 ! \times 2^{3}} \sum_{e_{I} \cap e_{J} \cap e_{K}=v} \varrho\left(e_{I}, e_{J}, e_{K}\right) \epsilon_{i j k} J_{e_{I}}^{i} J_{e_{J}}^{j} J_{e_{K}}^{k}\right|} \cdot f_{\gamma},
$$

where $\varrho\left(e_{I}, e_{J}, e_{K}\right):=\epsilon_{a b c} \varrho\left(e_{I}, S^{a}\right) \varrho\left(e_{J}, S^{b}\right) \varrho\left(e_{K}, S^{c}\right)$. Notice that the action of the regulated operator only depends on the properties of these surfaces at $v$. Hence the result is unchanged as we refine the partition and shrink $C$ to $v$ and hence the limit $\epsilon \rightarrow 0$ is trivial. However, the limiting volume operator carries the information of our choice of partitions through $\varrho\left(e_{I}, e_{J}, e_{K}\right)$, which depend on the background structure-the coordinates choice defining $S^{a}$. By suitable averaging over relevant background structures in (4.2), the well-defined, background-independent volume operator reads ${ }^{5}$

$$
\hat{V}_{\gamma}(R) \cdot f_{\gamma}=\ell_{\mathrm{p}}^{3} \beta^{\frac{3}{2}} \sum_{v \in V(\gamma) \cap R} \sqrt{\left|\frac{1}{3 ! \times 2^{3}} \sum_{e_{I} \cap e_{J} \cap e_{K}=v} \varsigma\left(e_{I}, e_{J}, e_{K}\right) \epsilon_{i j k} J_{e_{I}}^{i} J_{e_{J}}^{j} J_{e_{K}}^{k}\right|} \cdot f_{\gamma},
$$

where $\varsigma\left(e_{I}, e_{J}, e_{K}\right) \equiv \operatorname{sgn}\left(\operatorname{det}\left(\dot{e}_{I}(0), \dot{e}_{J}(0), \dot{e}_{K}(0)\right)\right)$ takes the values of $0,+1$ and -1 , corresponding to whether the determinant of the matrix formed by the tangents of the three edges at $v$ in that sequence is zero, positive, or negative.

\subsection{The matrix elements of the volume operator}

The volume operator acts on a spin-network state as

$$
\hat{V} \cdot T_{\gamma, \vec{j}, \vec{i}}(A)=\ell_{\mathrm{p}}^{3} \beta^{\frac{3}{2}} \sum_{v \in V(\gamma)} \sqrt{\left|\frac{i}{8 \times 4} \sum_{I<J<K, e_{I} \cap e_{J} \cap e_{K}=v} \varsigma\left(e_{I}, e_{J}, e_{K}\right) \hat{q}_{I J K}\right|} \cdot T_{\gamma, \vec{j}, \vec{i}}(A),
$$

where

$$
\begin{aligned}
\hat{q}_{I J K} & :=-4 i \epsilon_{i j k} J_{e_{I}}^{i} J_{e_{J}}^{j} J_{e_{K}}^{k}=4\left[\delta_{i j} J_{e_{I}}^{i} J_{e_{J}}^{j}, \delta_{l k} J_{e_{J}}^{l} J_{e_{K}}^{k}\right]=-\frac{1}{4}\left(16 \delta_{l k} J_{e_{J}}^{l} J_{e_{K}}^{k} \delta_{i j} J_{e_{I}}^{i} J_{e_{J}}^{j}-16 \delta_{i j} J_{e_{I}}^{i} J_{e_{J}}^{j} \delta_{l k} J_{e_{J}}^{l} J_{e_{K}}^{k}\right) \\
& =:-\frac{1}{4}\left(\hat{q}_{I J K}^{<J K ; I J>}-\hat{q}_{I J K}^{<I J ; J K>}\right) .
\end{aligned}
$$

Here $\delta_{i j}:=-2 \operatorname{tr}\left(\tau_{i} \tau_{j}\right)$ is the Cartan-Killing metric on $S U(2)$. The action of the volume operator is local, in the sense that its action is a sum on independent vertices. Therefore, we can focus on its action on a single vertex. The fact that the pseudo-vertices are divalent and the self-adjoint operators $J_{e_{I}}^{i}$ act only at the beginning points of $e_{I}$ implies that the summation in Eq. (4.5) is only over the true vertices $v$ of $\gamma$.

Equation (3.4) reveals the fact that the operators $\hat{q}_{I J K}$ and thus $\hat{V}$ only change the intertwiners $\vec{i}$ in $T_{\gamma, \vec{j}, \vec{i}}(A)$. The operators $\hat{q}_{I J K}$ acts on an intertwiner by contracting the corresponding matrix elements of $\tau_{i}$ with the intertwiner. Note that

$$
\begin{aligned}
\delta_{i j} J_{e_{I}}^{i} J_{e_{J}}^{j} \cdot\left(i_{v}^{J} ; \vec{a}\right)_{m_{1} \cdots m_{I} \cdots m_{J} \cdots m_{n}}^{M} & =\left(i_{v}^{J ; \vec{a}}\right)_{m_{1} \cdots m_{I}^{\prime} \cdots m_{J}^{\prime} \cdots m_{n}}{ }^{M}\left(-\left[\pi_{j_{I}}\left(\tau_{i}\right)\right]^{m_{I}^{\prime}}{ }_{m_{I}} \delta^{i j}\left[\pi_{j_{J}}\left(\tau_{j}\right)\right]^{m_{J}^{\prime}}{ }_{m_{J}}\right) \\
& =\left(i_{v}^{J ; \vec{a}}\right)_{m_{1} \cdots m_{I}^{\prime} \cdots m_{J}^{\prime} \cdots m_{n}}{ }^{M}\left[\pi_{j_{I}}\left(\tau_{\mu}\right)\right]_{m_{I}}^{m_{I}^{\prime}} C_{(1)}^{v \mu}\left[\pi_{j_{J}}\left(\tau_{\nu}\right)\right]^{m_{J}^{\prime}}{ }_{m_{J}} . \\
& =-C_{\mu \nu}^{(1)} J_{e_{I}}^{\mu} J_{e_{J}}^{v} \cdot\left(i_{v}^{J ; \vec{a}}\right)_{m_{1} \cdots m_{I} \cdots m_{J} \cdots m_{n}}^{M},
\end{aligned}
$$

where in the second step we have used the following identity (see Appendix B.1 for a proof):

$-\left[\pi_{j_{I}}\left(\tau_{i}\right)\right]^{m_{I}^{\prime}}{ }_{m_{I}} \delta^{i j}\left[\pi_{j_{J}}\left(\tau_{j}\right)\right]^{m_{J}^{\prime}}{ }_{m_{J}}=\left[\pi_{j_{I}}\left(\tau_{\mu}\right)\right]^{m_{I}^{\prime}}{ }_{m_{I}} C_{(1)}^{v \mu}\left[\pi_{j_{J}}\left(\tau_{v}\right)\right]^{m_{J}^{\prime}}{ }_{m_{J}}$.

\footnotetext{
${ }^{5}$ An overall undetermined multiplicative constant $\kappa_{\mathrm{av}}$ was fixed as 1 by the consistency check of volume and triad operator quantizations [45,46].
} 
Hence the operator $\hat{q}_{I J K}^{<J K ; I J>}$ and $\hat{q}_{I J K}^{<I J ; J>}$ in (4.6) can be represented in terms of $J^{\mu}$ by

$$
\begin{aligned}
& \hat{q}_{I J K}^{<J K ; I J>} \cdot\left(i_{v}^{J ; \vec{a}}\right)_{m_{1} \cdots m_{I} \cdots m_{J} \cdots m_{K} \cdots m_{n}}^{M}=16 C_{\rho \sigma}^{(1)} J_{e_{J}}^{\rho} J_{e_{K}}^{\sigma} C_{\mu \nu}^{(1)} J_{e_{I}}^{\mu} J_{e_{J}}^{\nu} \cdot\left(i_{v}^{J ; \vec{a}}\right)_{m_{1} \cdots m_{I} \cdots m_{J} \cdots m_{K} \cdots m_{n}}^{M} \\
& =16\left(i_{v}^{J ; \vec{a}}\right)_{m_{1} \cdots m_{I}^{\prime} \cdots m_{J}^{\prime} \cdots m_{K}^{\prime} \cdots m_{n}}{ }^{M}\left[\pi_{j_{J}}\left(\tau_{v}\right)\right]^{m_{J}^{\prime \prime}}{ }_{m_{J}} C_{(1)}^{v^{\prime} v}\left[\pi_{j_{K}}\left(\tau_{v^{\prime}}\right)\right]^{m_{K}^{\prime}}{ }_{m_{K}} \\
& \times\left[\pi_{j_{I}}\left(\tau_{\mu}\right)\right]_{m_{I}}^{m_{I}^{\prime}} C_{(1)}^{\mu^{\prime} \mu}\left[\pi_{j_{J}}\left(\tau_{\mu^{\prime}}\right)\right]_{m_{J}^{\prime \prime}}^{m_{J}^{\prime}}, \\
& \hat{q}_{I J K}^{<I J ; J K>} \cdot\left(i_{v}^{J ; \vec{a}}\right)_{m_{1} \cdots m_{I} \cdots m_{J} \cdots m_{K} \cdots m_{n}}^{M}=16 C_{\mu \nu}^{(1)} J_{e_{I}}^{\mu} J_{e_{J}}^{\nu} C_{\rho \sigma}^{(1)} J_{e_{J}}^{\rho} J_{e_{K}}^{\sigma} \cdot\left(i_{v}^{J ; \vec{a}}\right)_{m_{1} \cdots m_{I} \cdots m_{J} \cdots m_{K} \cdots m_{n}}^{M} \\
& =16\left(i_{v}^{J ; \vec{a}}\right)_{m_{1} \cdots m_{I}^{\prime} \cdots m_{J}^{\prime} \cdots m_{K}^{\prime} \cdots m_{n}}^{M}\left[\pi_{j_{I}}\left(\tau_{\mu}\right)\right]_{m_{I}}^{m_{I}^{\prime}} C_{(1)}^{\mu^{\prime} \mu}\left[\pi_{j_{J}}\left(\tau_{\mu^{\prime}}\right)\right]^{m_{J}^{\prime \prime}} m_{J} \\
& \times\left[\pi_{j_{J}}\left(\tau_{v}\right)\right]_{m_{J}^{\prime \prime}}^{m_{J}^{\prime}} C_{(1)}^{v^{\prime} v}\left[\pi_{j_{K}}\left(\tau_{v^{\prime}}\right)\right]_{m_{K}}^{m_{K}^{\prime}} .
\end{aligned}
$$

With the above preparation, we now turn to the action of $\hat{q}_{I J K}$ on the intertwiner $\left(i_{v}^{J ; \vec{a}}\right)_{m_{1} \cdots m_{I} \cdots m_{J} \cdots m_{K} \cdots m_{n}}{ }^{M}$ associated to a true vertex $v$ in the graphical method. We first consider the case $I>2$ and $K<n$, where $a_{I-1}$ and $a_{K}$ will appear in the final result. The other special cases will be dealt with later. According to Eq. (4.9), the first term in the parentheses of Eq. (4.6) evaluated on the intertwiner (3.14) can be represented by the following graphical formula (we present only the parts of the graph of the intertwiner which closely connect to the key steps in the following calculations):

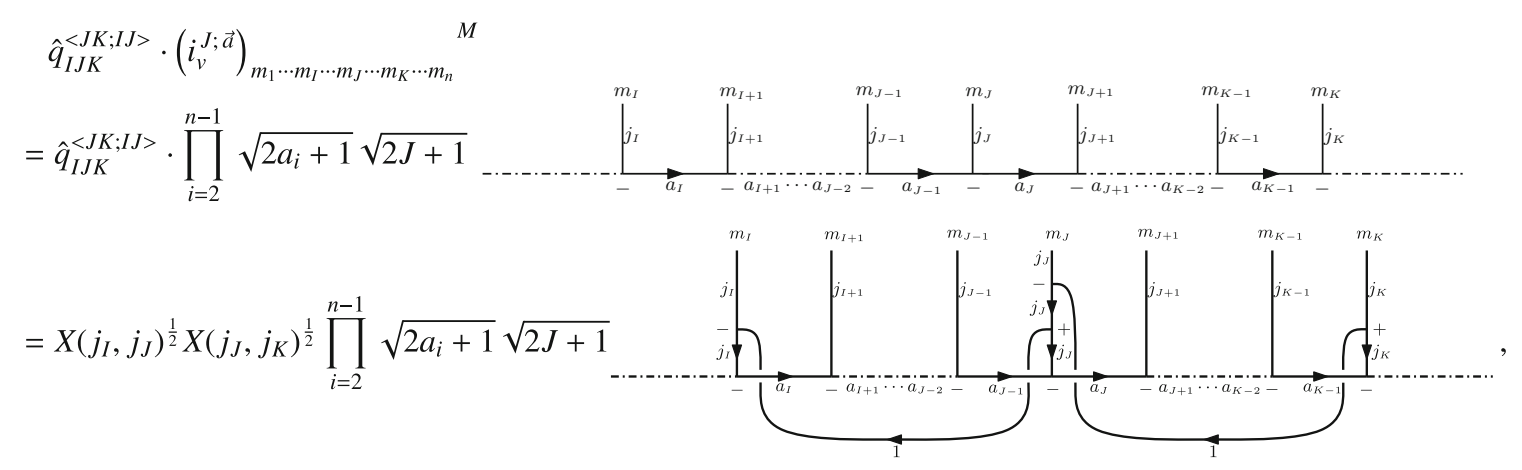

where $X\left(j_{1}, j_{2}\right) \equiv 2 j_{1}\left(2 j_{1}+1\right)\left(2 j_{1}+2\right) 2 j_{2}\left(2 j_{2}+1\right)\left(2 j_{2}+2\right)$. Similarly, the second term in the parentheses of Eq. (4.6) acting on the intertwiner can be expressed as

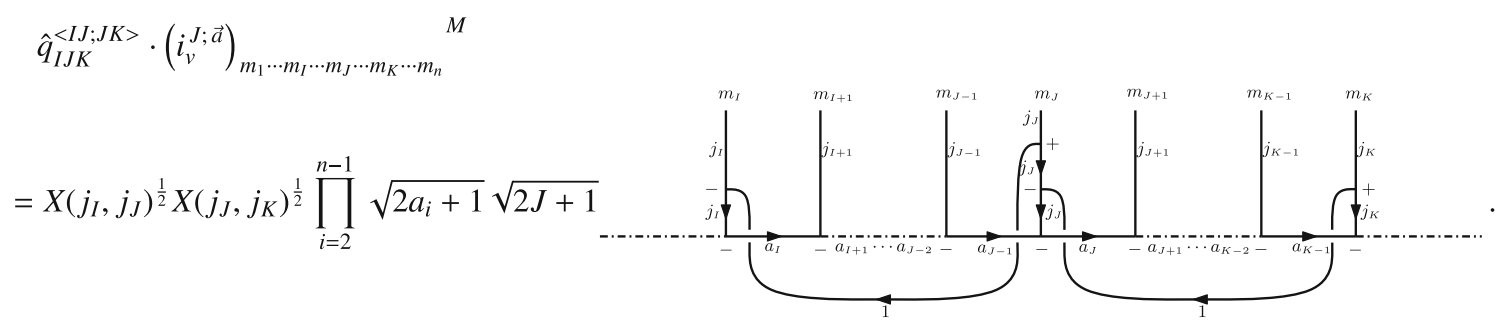

It is obvious that the two terms $\hat{q}_{I J K}^{<J K ; I J>}$ and $\hat{q}_{I J K}^{<I J ; J K>}$ in (4.6) are gauge invariant. Hence the operator $\hat{q}_{I J K}$ and the volume operator (4.5) are gauge invariant. Each of them leaves the intertwiner space $\mathcal{H}_{j_{1}, \ldots, j_{n}}^{v}$ with the intertwiners as its orthonormal basis, determined by the given $j_{1}, \ldots, j_{n}$ and the resulting angular momentum $J$, at the vertex $v$ invariant. Therefore the action of $\hat{q}_{I J K}$ on an intertwiner can be expanded linearly in terms of intertwiners in $\mathcal{H}_{j_{1}, \ldots, j_{n}}^{v}$ at the vertex $v$. In graphical language, they leave the vertical lines denoted by $j_{i}$ and the last horizontal line denoted by $J$ invariant, but change the intermediate couplings $a_{i}$ labeling the intermediate horizontal lines. Hence, in graphical calculation, our task is to drag the endpoints of the two curves with spin 1 down to attach the horizontal lines, and then yank them away from the horizontal lines 
following the simple and rigorous rules of transforming graphs presented in Appendix A.2. Of course, there are alternative ways, corresponding to different choices of coupling, to remove the two curves with spin 1 . The results obtained from different ways are related by unitary transformations. In the following, we choose a way guided by the simplicity principle that the number of changed intermediate values $a_{i}$ is as little as possible and the final result is as simple as possible. The calculations of the action of $\hat{q}_{I J K}$ on a given intertwiner in the graphical method consist of four steps. We first consider the case that $J>I+1$ and $K>J+1$ (the other cases will be handled later) and focus on the action of $\hat{q}_{I J K}^{<J K ; I J>}$ on $\left(i_{v}^{J ; \vec{a}}\right)_{m_{1} \cdots m_{I} \cdots m_{J} \cdots m_{K} \cdots m_{n}}{ }^{M}$. The four steps of our calculation are as follows (see Sect. 4 in [47] for details):

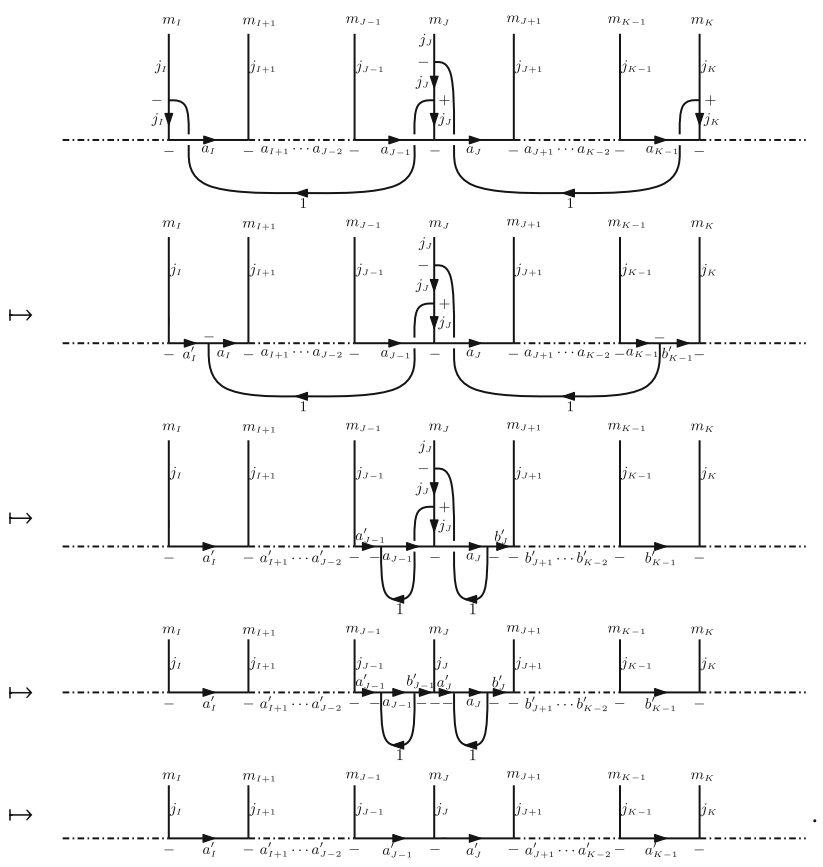

In the first step, we have been dragging the two endpoints of curves with spin 1 attached to lines with spins $j_{I}$ and $j_{K}$, respectively, down to join with two horizontal lines denoted by spins $a_{I}$ and $a_{K-1}$ by the following recoupling identities (see Appendix B.2 for a proof):

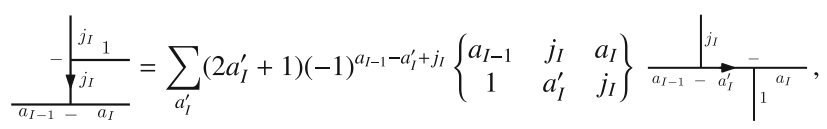

$\frac{1 \underbrace{j_{K}}_{\frac{a_{K-1}-a_{K}}{j_{K}}}}{a_{b_{K-1}}^{\prime}}\left(2 b_{K-1}^{\prime}+1\right)(-1)^{a_{K}-b_{K-1}^{\prime}+j_{K}+1}\left\{\begin{array}{ccc}a_{K} & j_{K} & a_{K-1} \\ 1 & b_{K-1}^{\prime} & j_{K}\end{array}\right\} \stackrel{j^{j_{K}}}{\underset{a_{K-1}-a_{K}}{b_{1}^{\prime}}}$.

In the second step, we have moved the two points labeled by $\left(a_{I}^{\prime}, a_{I}, 1\right)$ and $\left(a_{K-1}, b_{K-1}^{\prime}, 1\right)$, step by step, to the right-hand side of $\left(a_{J-2}, j_{J-1}, a_{J-1}\right)$ and the left-hand side of $\left(a_{J}, j_{J+1}, a_{J+1}\right)$, respectively, by repeatedly applying of the following identities (see Appendix B.2 for a proof):

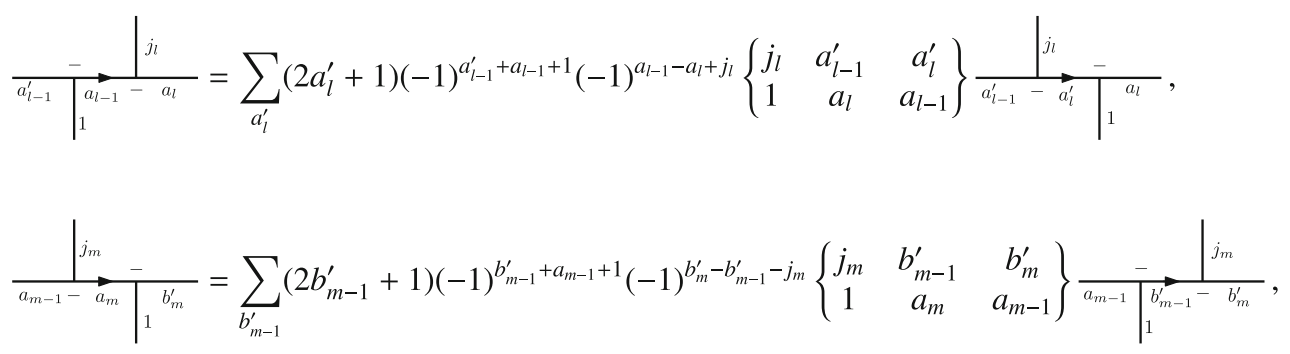


where $l=I+1, \ldots, J-1$ and $m=K-1, \ldots, J+1$. In the third step, the identities (4.15) and (4.14) were used again to drag the two endpoints of two curves with spin 1 attached to the line with spin $j_{J}$ down to join with two horizontal lines denoted by spins $a_{J-1}$ and $a_{J}$, respectively. In the fourth (last) step, the two curves with spin 1 were removed from intertwiners by the identity (see Appendix B.3 for a proof)

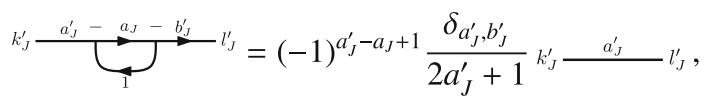

and we have summed over $b_{J-1}^{\prime}$ and $a_{J}^{\prime}$ and relabeled the indices $b^{\prime}$ by $a^{\prime}$. Hence the action of the operator $\hat{q}_{I J K}^{<J K ; I J>}$ on the intertwiner $\left(i_{v}^{J ; \vec{a}}\right)_{m_{1} \cdots m_{n}}{ }^{M}$ reads

$$
\begin{aligned}
& \hat{q}_{I J K}^{<J K ; I J>} \cdot\left(i_{v}^{J ; \vec{a}}\right)_{m_{1} \cdots m_{I} \cdots m_{J} \cdots m_{K} \cdots m_{n}} \\
& \begin{array}{c}
=\sum_{\left(a_{I}^{\prime}, \ldots, a_{K-1}^{\prime}\right)}\left(2 a_{I}^{\prime}+1\right)(-1)^{a_{I-1}-a_{I}^{\prime}+j_{I}}\left\{\begin{array}{ccc}
a_{I-1} & j_{I} & a_{I} \\
1 & a_{I}^{\prime} & j_{I}
\end{array}\right\} \\
\times\left(2 a_{K-1}^{\prime}+1\right)(-1)^{a_{K}-a_{K-1}^{\prime}+j_{K}+1}\left\{\begin{array}{ccc}
a_{K} & j_{K} & a_{K-1} \\
1 & a_{K-1}^{\prime} & j_{K}
\end{array}\right\}
\end{array}
\end{aligned}
$$

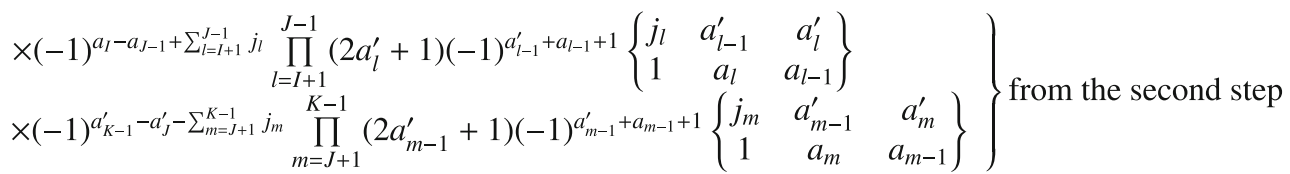

$$
\begin{aligned}
& \left.\times\left\{\begin{array}{ccc}
a_{J} & j_{J} & a_{J-1} \\
1 & a_{J-1}^{\prime} & j_{J}
\end{array}\right\}(-1)^{a_{J}-a_{J-1}^{\prime}+j_{J}+1}(-1)^{-a_{J-1}^{\prime}+a_{J-1}-1}\right\} \\
& \left.\times\left\{\begin{array}{ccc}
a_{J-1}^{\prime} & j_{J} & a_{J} \\
1 & a_{J}^{\prime} & j_{J}
\end{array}\right\}(-1)^{a_{J-1}^{\prime}-a_{J}^{\prime}+j_{J}}(-1)^{a_{J}^{\prime}-a_{J}+1} \quad\right\} \text { from the third and forth steps }
\end{aligned}
$$

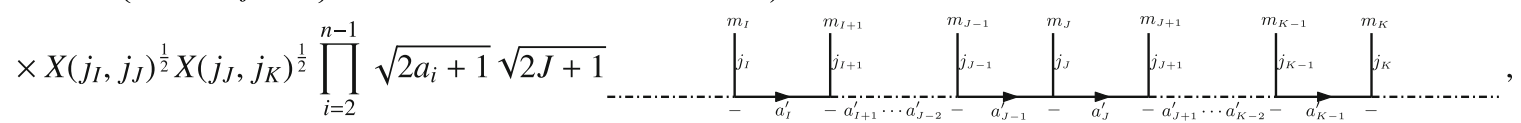

which is a linear combination of new intertwiners. The expression (4.19) can be simplified in two aspects. One is to get more symmetric factors in the two multi-products. The other is to simplify the exponents. Notice that the result (4.19) was obtained in the case of $J>I+1$ and $K>J+1$. Under this case, the product terms of $\left(2 a^{\prime}+1\right)$ can be reduced to

$$
\left(2 a_{K-1}^{\prime}+1\right) \prod_{m=J+1}^{K-1}\left(2 a_{m-1}^{\prime}+1\right)=\left(2 a_{J}^{\prime}+1\right) \prod_{m=J+1}^{K-1}\left(2 a_{m}^{\prime}+1\right) .
$$

This expression enables us to write the multiple product over $m$ as the formula which is closer to the multiple product over $l$ in Eq. (4.19). By simplifying the exponents and properly adjusting the ordering of multi-products of $\sqrt{2 a+1}$, we finally obtain the compact result

$$
\begin{aligned}
& \hat{q}_{I J K}^{<J K ; I J>} \cdot\left(i_{v}^{J ; \vec{a}}\right)_{m_{1} \cdots m_{I} \cdots m_{J} \cdots m_{K} \cdots m_{n}} M \\
& =\sum_{\left(a_{I}^{\prime}, \ldots, a_{K-1}^{\prime}\right)}(-1)^{a_{I-1}+j_{I}+a_{K}+j_{K}}(-1)^{a_{I}-a_{I}^{\prime}}(-1)^{\sum_{l=I+1}^{J-1} j_{l}}(-1)^{-\sum_{m=J+1}^{K-1} j_{m}} X\left(j_{I}, j_{J}\right)^{\frac{1}{2}} X\left(j_{J}, j_{K}\right)^{\frac{1}{2}} \\
& \times \sqrt{\left(2 a_{I}^{\prime}+1\right)\left(2 a_{I}+1\right)} \sqrt{\left(2 a_{J}^{\prime}+1\right)\left(2 a_{J}+1\right)} \times\left\{\begin{array}{ccc}
a_{I-1} & j_{I} & a_{I} \\
1 & a_{I}^{\prime} & j_{I}
\end{array}\right\}\left\{\begin{array}{ccc}
a_{K} & j_{K} & a_{K-1} \\
1 & a_{K-1}^{\prime} & j_{K}
\end{array}\right\} \\
& \times \prod_{l=I+1}^{J-1} \sqrt{\left(2 a_{l}^{\prime}+1\right)\left(2 a_{l}+1\right)}(-1)^{a_{l-1}^{\prime}+a_{l-1}+1} \times\left\{\begin{array}{ccc}
j_{l} & a_{l-1}^{\prime} & a_{l}^{\prime} \\
1 & a_{l} & a_{l-1}
\end{array}\right\}
\end{aligned}
$$




$$
\begin{aligned}
& \times \prod_{m=J+1}^{K-1} \sqrt{\left(2 a_{m}^{\prime}+1\right)\left(2 a_{m}+1\right)}(-1)^{a_{m-1}^{\prime}+a_{m-1}+1}\left\{\begin{array}{ccc}
j_{m} & a_{m-1}^{\prime} & a_{m}^{\prime} \\
1 & a_{m} & a_{m-1}
\end{array}\right\}(-1)^{a_{J-1}^{\prime}+a_{J}^{\prime}} \\
& \times\left\{\begin{array}{ccc}
a_{J} & j_{J} & a_{J-1} \\
1 & a_{J-1}^{\prime} & j_{J}
\end{array}\right\}\left\{\begin{array}{ccc}
a_{J-1}^{\prime} & j_{J} & a_{J} \\
1 & a_{J}^{\prime} & j_{J}
\end{array}\right\}\left(i_{v}^{J ;}\right)_{m_{1} \cdots m_{I} \cdots m_{J} \cdots m_{K} \cdots m_{n}},
\end{aligned}
$$

where the tuple $\overrightarrow{\tilde{a}}$ in the new intertwiner $\left(i_{v}^{J ;} \overrightarrow{\tilde{a}}\right)_{m_{1} \cdots m_{I} \cdots m_{J} \cdots m_{K} \cdots m_{n}}^{M}$ is given by $\overrightarrow{\tilde{a}}:=\left\{a_{2}, \ldots, a_{I-1}, a_{I}^{\prime}, \ldots, a_{K-1}^{\prime}, a_{K}, \ldots\right.$, $\left.a_{n-1}\right\}$. The above result is in the case of $J>I+1$ and $K>J+1$, which ensures that the limitations of the summations and multi-product exist, namely, the upper limitations are always not smaller than the low limitations. Intuitively the results for the other cases can be thought of as special cases of the above result by omitting the corresponding summations and multiplications which do not exist. A detailed analysis in step by step confirms this intuition (see Sect. 4 in [47] for a discussion).

The second term in the parentheses of Eq. (4.6) can be calculated similarly to the former one. Here we omit the intermediate steps and directly write down the result as (a complete calculation is also shown in Appendix C in [47])

$$
\begin{aligned}
& \hat{q}_{I J K}^{<I J ; J K>} \cdot\left(i_{v}^{J ; \vec{a}}\right)_{m_{1} \cdots m_{I} \cdots m_{J} \cdots m_{K} \cdots m_{n}}^{M}
\end{aligned}
$$

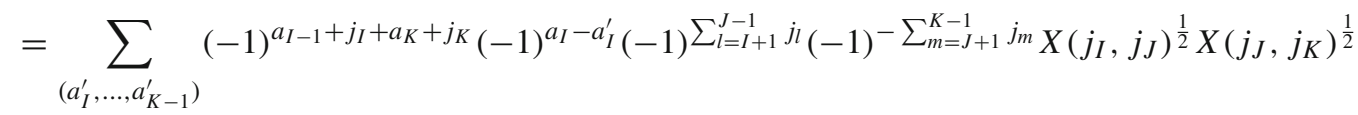

$$
\begin{aligned}
& \times \sqrt{\left(2 a_{I}^{\prime}+1\right)\left(2 a_{I}+1\right)} \sqrt{\left(2 a_{J}^{\prime}+1\right)\left(2 a_{J}+1\right)}\left\{\begin{array}{ccc}
a_{I-1} & j_{I} & a_{I} \\
1 & a_{I}^{\prime} & j_{I}
\end{array}\right\}\left\{\begin{array}{ccc}
a_{K} & j_{K} & a_{K-1} \\
1 & a_{K-1}^{\prime} & j_{K}
\end{array}\right\} \\
& \times \prod_{l=I+1}^{J-1} \sqrt{\left(2 a_{l}^{\prime}+1\right)\left(2 a_{l}+1\right)}(-1)^{a_{l-1}^{\prime}+a_{l-1}+1}\left\{\begin{array}{ccc}
j_{l} & a_{l-1}^{\prime} & a_{l}^{\prime} \\
1 & a_{l} & a_{l-1}
\end{array}\right\} \\
& \times \prod_{m=J+1}^{K-1} \sqrt{\left(2 a_{m}^{\prime}+1\right)\left(2 a_{m}+1\right)}(-1)^{a_{m-1}^{\prime}+a_{m-1}+1}\left\{\begin{array}{ccc}
j_{m} & a_{m-1}^{\prime} & a_{m}^{\prime} \\
1 & a_{m} & a_{m-1}
\end{array}\right\}(-1)^{a_{J-1}+a_{J}}\left\{\begin{array}{cccc}
a_{J}^{\prime} & j_{J} & a_{J-1} \\
1 & a_{J-1}^{\prime} & j_{J}
\end{array}\right\}\left\{\begin{array}{ccc}
a_{J-1} & j_{J} & a_{J} \\
1 & a_{J}^{\prime} & j_{J}
\end{array}\right\} \\
& \times\left(i_{v}^{J ; \overrightarrow{\tilde{a}}}\right)_{m_{1} \cdots m_{I} \cdots m_{J} \cdots m_{K} \cdots m_{n}}^{M} .
\end{aligned}
$$

The final results for the special cases of $J-1<I+1$ and $K-1<J+1$ can be obtained from (4.22) by omitting the corresponding multi-products $\prod_{l=I+1}^{J-1}$ and $\prod_{m=J+1}^{K-1}$ and summations $\sum_{l=I+1}^{J-1}$ and $\sum_{m=J+1}^{K-1}$. Combining the results (4.21) with (4.22), for the case of $I>2$ and $K<n$, the action of $\hat{q}_{I J K}$ in Eq. (4.6) on the intertwiner can be explicitly written down as

$$
\begin{aligned}
& \hat{q}_{I J K} \cdot\left(i_{v}^{J ; \vec{a}}\right)_{m_{1} \cdots m_{I} \cdots m_{J} \cdots m_{K} \cdots m_{n}}^{M} \\
& =-\frac{1}{4} \sum_{\left(a_{I}^{\prime}, \ldots, a_{K-1}^{\prime}\right)}(-1)^{a_{K}+j_{K}+a_{I-1}+j_{I}}(-1)^{a_{I}-a_{I}^{\prime}}(-1)^{\sum_{l=I+1}^{J-1} j_{l}}(-1)^{-\sum_{m=J+1}^{K-1} j_{m}} X\left(j_{I}, j_{J}\right)^{\frac{1}{2}} X\left(j_{J}, j_{K}\right)^{\frac{1}{2}} \\
& \times \sqrt{\left(2 a_{I}^{\prime}+1\right)\left(2 a_{I}+1\right)} \sqrt{\left(2 a_{J}^{\prime}+1\right)\left(2 a_{J}+1\right)}\left\{\begin{array}{ccc}
a_{I-1} & j_{I} & a_{I} \\
1 & a_{I}^{\prime} & j_{I}
\end{array}\right\}\left\{\begin{array}{ccc}
a_{K} & j_{K} & a_{K-1} \\
1 & a_{K-1}^{\prime} & j_{K}
\end{array}\right\} \\
& \times \prod_{l=I+1}^{J-1} \sqrt{\left(2 a_{l}^{\prime}+1\right)\left(2 a_{l}+1\right)}(-1)^{a_{l-1}^{\prime}+a_{l-1}+1}\left\{\begin{array}{ccc}
j_{l} & a_{l-1}^{\prime} & a_{l}^{\prime} \\
1 & a_{l} & a_{l-1}
\end{array}\right\} \\
& \times \prod_{m=J+1}^{K-1} \sqrt{\left(2 a_{m}^{\prime}+1\right)\left(2 a_{m}+1\right)}(-1)^{a_{m-1}^{\prime}+a_{m-1}+1}\left\{\begin{array}{ccc}
j_{m} & a_{m-1}^{\prime} & a_{m}^{\prime} \\
1 & a_{m} & a_{m-1}
\end{array}\right\} \\
& \times\left[(-1)^{a_{J-1}^{\prime}+a_{J}^{\prime}}\left\{\begin{array}{ccc}
a_{J} & j_{J} & a_{J-1} \\
1 & a_{J-1}^{\prime} & j_{J}
\end{array}\right\}\left\{\begin{array}{ccc}
a_{J-1}^{\prime} & j_{J} & a_{J} \\
1 & a_{J}^{\prime} & j_{J}
\end{array}\right\}-(-1)^{a_{J-1}+a_{J}}\left\{\begin{array}{ccc}
a_{J}^{\prime} & j_{J} & a_{J-1} \\
1 & a_{J-1}^{\prime} & j_{J}
\end{array}\right\}\left\{\begin{array}{ccc}
a_{J-1} & j_{J} & a_{J} \\
1 & a_{J}^{\prime} & j_{J}
\end{array}\right\}\right] \\
& \times\left(i_{v}^{J ; \overrightarrow{\tilde{a}}}\right)_{m_{1} \cdots m_{I} \cdots m_{J} \cdots m_{K} \cdots m_{n}}^{M} .
\end{aligned}
$$


Again, we expect that the result (4.23) is general and also suitable for the remaining special cases of $0<I \leqslant 2$ and $K=n$ when $a_{I-1}$ and $a_{K}$ do not exist. While $a_{I-1}$ and $a_{K}$ do not exist in the intertwiner, we can "create" them via the formula (see Appendix B.3 for a proof)

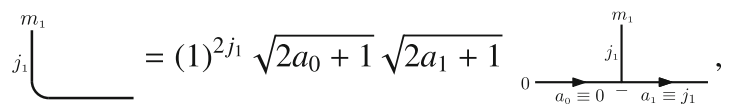

where $a_{0} \equiv 0, a_{1} \equiv j_{1}$, and $J$ is relabeled by $a_{n}$. Then the intertwiner in (3.14) can be extended as

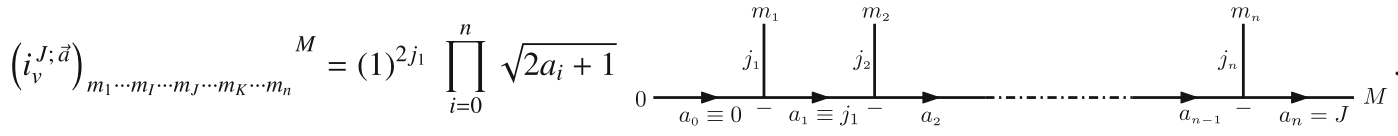

We are immediately awake to the fact that (4.23) is suitable for these special cases just by taking $a_{0}=0, a_{1}=a_{1}^{\prime}=j_{1}$, and $a_{n}=J$.

Note that the operator $\hat{q}_{I J K}$ at $v$ only changes the intermediate angular momenta $a_{I}, \ldots, a_{K-1}$ between $I$ and $K$ of the intertwiner $i_{v}$, but leaves the angular momenta $j_{1}, \ldots, j_{n}, J$ and the magnetic quantum numbers $m_{1}, \ldots, m_{n}, M$ invariant. The matrix elements of $\hat{q}_{I J K}$ with respect to two given normalized spin-network states $T_{\gamma, \vec{j}, \vec{i}}^{\mathrm{norm}}(A):=\prod_{e \in E(\gamma)} \sqrt{2 j_{e}+1} T_{\gamma, \vec{j}, \vec{i}}(A)$ read

$$
\begin{aligned}
\left(T_{\gamma^{\prime}, \vec{j}^{\prime}, \vec{i}^{\prime}}^{\text {norm }}, \hat{q}_{I J K} \cdot T_{\gamma, \vec{j}, \vec{i}}^{\text {norm }}\right)_{\mathcal{H}_{\text {kin }}} & =\int_{S U(2)} \prod_{E(\tilde{\gamma}) \mid} \prod_{e \in E(\tilde{\gamma})} \mathrm{d} \mu_{H}\left(h_{e}\right) \overline{T_{\gamma^{\prime}, \vec{j}^{\prime}, \vec{i}^{\prime}}^{\text {norm }}(A)} \hat{q}_{I J K} \cdot T_{\gamma, \vec{j}, \vec{i}}^{\text {norm }}(A) \\
& =\delta_{\gamma, \gamma^{\prime}} \int_{S U(2)|E(\gamma)|} \prod_{e \in E(\gamma)} \mathrm{d} \mu_{H}\left(h_{e}\right) \overline{T_{\gamma, \vec{j}^{\prime}, \vec{i}^{\prime}}^{\text {norm }}(A)} \hat{q}_{I J K} \cdot T_{\gamma, \vec{j}, \vec{i}}^{\text {norm }}(A) \\
& =\delta_{\gamma, \gamma^{\prime}} \prod_{e \in E(\gamma)} \delta_{j_{e}, j_{e}^{\prime}} \prod_{v \in V(\gamma)} \sum_{m_{1}, \ldots, m_{n}} \overline{\left(i_{v}^{J^{\prime} ; \vec{a}^{\prime}}\right)_{m_{1} \cdots m_{n}}{ }^{M^{\prime}}} \hat{q}_{I J K} \cdot\left(i_{v}^{J} ; \vec{a}\right)_{m_{1} \cdots m_{n}} \\
& =\delta_{\gamma, \gamma^{\prime}} \prod_{e \in E(\gamma)} \delta_{j_{e}, j_{e}^{\prime}} \prod_{v \in V(\gamma)}\left\langle\vec{a}^{\prime} ; J^{\prime}, M^{\prime}\left|\hat{q}_{I J K}\right| \vec{a} ; J, M\right\rangle_{\mathcal{H}_{j_{1}, \ldots, j_{n}}^{v}} \\
& =: \delta_{\gamma, \gamma^{\prime}} \prod_{e \in E(\gamma)} \delta_{j_{e}, j_{e}^{\prime}} \prod_{v \in V(\gamma)}\left\langle\vec{a}^{\prime}\left|\hat{q}_{I J K}\right| \vec{a}\right\rangle \delta_{J, J^{\prime}} \delta_{M, M^{\prime}} .
\end{aligned}
$$

In the second step of Eq. (4.26), we notice that $\hat{q}_{I J K}$ at $v$ only changes the intertwiner $i_{v}$, and the integration with respect to the Haar measure gives a zero result if $\gamma$ differs from $\gamma^{\prime}$. In the third step, the integration for the holonomies along the same edges but with different spins yields delta functions and the contractions of the intertwiner with its conjugate. In the fourth step, we have used the definition (A.23) of the inner product of the intertwiner space $\mathcal{H}_{j_{1}, \ldots, j_{n}}^{v}$ associated to $v$. Thus the general expression (4.23) allows us to uniformly write the matrix elements of $\hat{q}_{I J K}$ in the gauge-variant and gauge-invariant intertwiner, corresponding to resulting angular momentum $J \neq 0$ and $J=0$, respectively, as

$$
\begin{aligned}
\left\langle\vec{a}^{\prime}\left|\hat{q}_{I J K}\right| \vec{a}\right\rangle \equiv & \sum_{m_{1}, \ldots, m_{n}} \overline{\left(i_{v}^{J ; \vec{a}^{\prime}}\right)_{m_{1} \cdots m_{n}}{ }^{M}} \hat{q}_{I J K} \cdot\left(i_{v}^{J ;}\right)_{m_{1} \cdots m_{n}}{ }^{M}=-\frac{1}{4} \sum_{\left(a_{I}^{\prime \prime}, \ldots, a_{K-1}^{\prime \prime}\right)}(-1)^{a_{K}+j_{K}+a_{I-1}+j_{I}}(-1)^{a_{I}-a_{I}^{\prime \prime}} \\
& \times(-1)^{\sum_{l=I+1}^{J-1} j_{l}}(-1)^{-\sum_{m=J+1}^{K-1} j_{m}} X\left(j_{I}, j_{J}\right)^{\frac{1}{2}} X\left(j_{J}, j_{K}\right)^{\frac{1}{2}} \sqrt{\left(2 a_{I}^{\prime \prime}+1\right)\left(2 a_{I}+1\right)} \sqrt{\left(2 a_{J}^{\prime \prime}+1\right)\left(2 a_{J}+1\right)} \\
& \times\left\{\begin{array}{ccc}
a_{I-1} & j_{I} & a_{I} \\
1 & a_{I}^{\prime \prime} & j_{I}
\end{array}\right\}\left\{\begin{array}{ccc}
a_{K} & j_{K} & a_{K-1} \\
1 & a_{K-1}^{\prime \prime} & j_{K}
\end{array}\right\} \prod_{l=I+1}^{J-1} \sqrt{\left(2 a_{l}^{\prime \prime}+1\right)\left(2 a_{l}+1\right)}(-1)^{a_{l-1}^{\prime \prime}+a_{l-1}+1}\left\{\begin{array}{ccc}
j_{l} & a_{l-1}^{\prime \prime} & a_{l}^{\prime \prime} \\
1 & a_{l} & a_{l-1}
\end{array}\right\} \\
& \times \prod_{m=J+1}^{K-1} \sqrt{\left(2 a_{m}^{\prime \prime}+1\right)\left(2 a_{m}+1\right)}(-1)^{a_{m-1}^{\prime \prime}+a_{m-1}+1}\left\{\begin{array}{ccc}
j_{m} & a_{m-1}^{\prime \prime} & a_{m}^{\prime \prime} \\
1 & a_{m} & a_{m-1}
\end{array}\right\} \\
& \times\left[(-1)^{a_{J-1}^{\prime \prime}+a_{J}^{\prime \prime}}\left\{\begin{array}{ccc}
a_{J} & j_{J} & a_{J-1} \\
1 & a_{J-1}^{\prime \prime} & j_{J}
\end{array}\right\}\left\{\begin{array}{ccc}
a_{J-1}^{\prime \prime} & j_{J} & a_{J} \\
1 & a_{J}^{\prime \prime} & j_{J}
\end{array}\right\}-(-1)^{a_{J-1}+a_{J}}\left\{\begin{array}{ccc}
a_{J}^{\prime \prime} & j_{J} & a_{J-1} \\
1 & a_{J-1}^{\prime \prime} & j_{J}
\end{array}\right\}\left\{\begin{array}{ccc}
a_{J-1} & j_{J} & a_{J} \\
1 & a_{J}^{\prime \prime} & j_{J}
\end{array}\right\}\right]
\end{aligned}
$$




$$
\begin{aligned}
& \times \sum_{m_{1}, \ldots, m_{n}} \overline{\left(i_{v}^{J ; \vec{a}^{\prime}}\right)_{m_{1} \cdots m_{n}}^{M}}\left(i_{v}^{J ; \overrightarrow{\tilde{a}}^{\prime \prime}}\right)_{m_{1} \cdots m_{I} \cdots m_{J} \cdots m_{K} \cdots m_{n}}^{M}=-\frac{1}{4}(-1)^{a_{K}+j_{K}+a_{I-1}+j_{I}}(-1)^{a_{I}-a_{I}^{\prime}(-1)^{\sum_{l=I+1}^{J-1} j_{l}}} \\
& \times(-1)^{-\sum_{m=J+1}^{K-1} j_{m}} X\left(j_{I}, j_{J}\right)^{\frac{1}{2}} X\left(j_{J}, j_{K}\right)^{\frac{1}{2}} \sqrt{\left(2 a_{I}^{\prime}+1\right)\left(2 a_{I}+1\right)} \sqrt{\left(2 a_{J}^{\prime}+1\right)\left(2 a_{J}+1\right)} \\
& \times\left\{\begin{array}{ccc}
a_{I-1} & j_{I} & a_{I} \\
1 & a_{I}^{\prime} & j_{I}
\end{array}\right\}\left\{\begin{array}{ccc}
a_{K} & j_{K} & a_{K-1} \\
1 & a_{K-1}^{\prime} & j_{K}
\end{array}\right\} \prod_{l=I+1}^{J-1} \sqrt{\left(2 a_{l}^{\prime}+1\right)\left(2 a_{l}+1\right)}(-1)^{a_{l-1}^{\prime}+a_{l-1}+1}\left\{\begin{array}{ccc}
j_{l} & a_{l-1}^{\prime} & a_{l}^{\prime} \\
1 & a_{l} & a_{l-1}
\end{array}\right\} \\
& \times \prod_{m=J+1}^{K-1} \sqrt{\left(2 a_{m}^{\prime}+1\right)\left(2 a_{m}+1\right)}(-1)^{a_{m-1}^{\prime}+a_{m-1}+1}\left\{\begin{array}{ccc}
j_{m} & a_{m-1}^{\prime} & a_{m}^{\prime} \\
1 & a_{m} & a_{m-1}
\end{array}\right\} \\
& \times\left[(-1)^{a_{J-1}^{\prime}+a_{J}^{\prime}}\left\{\begin{array}{ccc}
a_{J} & j_{J} & a_{J-1} \\
1 & a_{J-1}^{\prime} & j_{J}
\end{array}\right\}\left\{\begin{array}{ccc}
a_{J-1}^{\prime} & j_{J} & a_{J} \\
1 & a_{J}^{\prime} & j_{J}
\end{array}\right\}-(-1)^{a_{J-1}+a_{J}}\left\{\begin{array}{ccc}
a_{J}^{\prime} & j_{J} & a_{J-1} \\
1 & a_{J-1}^{\prime} & j_{J}
\end{array}\right\}\left\{\begin{array}{cccc}
a_{J-1} & j_{J} & a_{J} \\
1 & a_{J}^{\prime} & j_{J}
\end{array}\right\}\right] \\
& \times\left\{\begin{array}{cl}
\prod_{s=2}^{I-1} \delta_{a_{s}^{\prime}, a_{s}} \prod_{t=K}^{n-1} \delta_{a_{t}^{\prime}, a_{t}}, & \text { for } I>2 \text { and } K<n \\
\prod_{s=2}^{I-1} \delta_{a_{s}^{\prime}, a_{s}}, & \text { for } I>2 \text { and } K=n \\
\prod_{t=K}^{n-1} \delta_{a_{t}^{\prime}, a_{t}}, & \text { for } I \leqslant 2 \text { and } K<n \\
1, & \text { for } I \leqslant 2 \text { and } K=n
\end{array},\right.
\end{aligned}
$$

where the tuple $\overrightarrow{\tilde{a}}^{\prime \prime}$ in $\left(i_{v}^{J ; \overrightarrow{\tilde{a}}^{\prime \prime}}\right)_{m_{1} \cdots m_{I} \cdots m_{J} \cdots m_{K} \cdots m_{n}}^{M}$ is given by $\overrightarrow{\tilde{a}}^{\prime \prime}:=\left\{a_{2}, \ldots, a_{I-1}, a_{I}^{\prime \prime}, \ldots, a_{K-1}^{\prime \prime}, a_{K}, \ldots, a_{n-1}\right\}$, and in the third step we have used the fact that intertwiners are orthonormal. Note that the multi-products $\prod_{l=I+1}^{J-1}$ and $\prod_{m=J+1}^{K-1}$ and summations $\sum_{l=I+1}^{J-1}$ and $\sum_{m=J+1}^{K-1}$ should be omitted for the cases of $J<I+2$ and $K<J+2$, and we need to set $a_{0}=0, a_{1}=a_{1}^{\prime}=j_{1}$, and $a_{n}=J$ when $0<I \leqslant 2$ and $K=n$, respectively. Under exchanging $a \leftrightarrow a^{\prime}$, the expression in the square bracket of (4.27) is antisymmetric, while the other terms are left invariant because of the symmetric properties of $6 j$-symbol, the symmetry of the delta function and the fact that $(-1)^{a_{I}-a_{I}^{\prime}}=(-1)^{a_{I}^{\prime}-a_{I}}$. Hence the matrix elements of $\hat{q}_{I J K}$ are antisymmetric, i.e.,

$\left\langle\vec{a}^{\prime}\left|\hat{q}_{I J K}\right| \vec{a}\right\rangle=-\left\langle\vec{a}\left|\hat{q}_{I J K}\right| \vec{a}^{\prime}\right\rangle$.

The matrix element formula (4.27) derived in graphical method is the same as the formula obtained from algebraic manipulation for the case of $I>1$ and $J>I+1$ in [19], although different ways were adopted to deal with the recoupling problem. Moreover, as shown above, the formula (4.27) is also valid for other cases and hence can be regarded as a general expression.

Finally, we consider some special cases which usually appear. With the following values of the $6 j$-symbols [41]:

$$
\begin{aligned}
& \left\{\begin{array}{lll}
0 & b & c \\
d & e & f
\end{array}\right\}=(-1)^{b+e+d} \frac{\delta_{b, c} \delta_{e, f}}{\sqrt{(2 b+1)(2 e+1)}}, \\
& \left\{\begin{array}{lll}
a & b & c \\
1 & c & b
\end{array}\right\}=(-1)^{s+1} \frac{2[b(b+1)+c(c+1)-a(a+1)]}{X(b, c)^{1 / 2}}, \\
& \left\{\begin{array}{ccc}
a & b & c-1 \\
1 & c & b
\end{array}\right\}=(-1)^{s}\left[\frac{2(s+1)(s-2 a)(s-2 b)(s-2 c+1)}{2 b(2 b+1)(2 b+2)(2 c-1) 2 c(2 c+1)}\right]^{1 / 2},
\end{aligned}
$$

where $s \equiv a+b+c$, the general matrix element formula (4.27) can be simplified in the following special cases.

(I) $I=1, J=2, K=3$

In this case, the general matrix element formula (4.27) reduces to

$$
\left\langle\vec{a}^{\prime}\left|\hat{q}_{123}\right| \vec{a}\right\rangle=-\frac{1}{2}(-1)^{j_{1}+j_{2}+j_{3}+a_{2}+a_{2}^{\prime}+a_{3}} X\left(j_{2}, j_{3}\right)^{\frac{1}{2}} \sqrt{\left(2 a_{2}^{\prime}+1\right)\left(2 a_{2}+1\right)}\left\{\begin{array}{ccc}
j_{1} & j_{2} & a_{2} \\
1 & a_{2}^{\prime} & j_{2}
\end{array}\right\}\left\{\begin{array}{ccc}
a_{3} & j_{3} & a_{2} \\
1 & a_{2}^{\prime} & j_{3}
\end{array}\right\}
$$




$$
\times\left[a_{2}^{\prime}\left(a_{2}^{\prime}+1\right)-a_{2}\left(a_{2}+1\right)\right] \times\left\{\begin{array}{cc}
\prod_{t=3}^{n-1} \delta_{a_{t}^{\prime}, a_{t},} & \text { for } n>3 \\
1, & \text { for } n=3
\end{array} .\right.
$$

Moreover, we can further simplify the result (4.32), since the triangular conditions on the $6 j$-symbols will constrain the values of $a^{\prime}$ in (4.32) as $a_{2}^{\prime} \in\left\{a_{2}-1, a_{2}, a_{2}+1\right\}$. Denoting $\left|a_{2}\right\rangle \equiv\left|a_{2}, a_{3}, \ldots\right\rangle$ and $\left|a_{2}-1\right\rangle \equiv\left|a_{2}-1, a_{3}, \ldots\right\rangle$, we get

$$
\begin{aligned}
& \left\langle a_{2}-1\left|\hat{q}_{123}\right| a_{2}\right\rangle=-\frac{1}{2}(-1)^{j_{1}+j_{2}+j_{3}+a_{2}+a_{2}-1+a_{3}} X\left(j_{2}, j_{3}\right)^{\frac{1}{2}} \\
& \times \sqrt{\left[2\left(a_{2}-1\right)+1\right]\left(2 a_{2}+1\right)}\left\{\begin{array}{ccc}
j_{1} & j_{2} & a_{2} \\
1 & a_{2}-1 & j_{2}
\end{array}\right\}\left\{\begin{array}{ccc}
a_{3} & j_{3} & a_{2} \\
1 & a_{2}-1 & j_{3}
\end{array}\right\} \\
& \times\left[\left(a_{2}-1\right)\left(a_{2}-1+1\right)-a_{2}\left(a_{2}+1\right)\right] \\
& =-\frac{1}{\sqrt{\left(2 a_{2}-1\right)\left(2 a_{2}+1\right)}}\left[\left(j_{1}+j_{2}+a_{2}+1\right)\left(-j_{1}+j_{2}+a_{2}\right)\left(j_{1}-j_{2}+a_{2}\right)\left(j_{1}+j_{2}-a_{2}+1\right)\right. \\
& \left.\times\left(a_{3}+j_{3}+a_{2}+1\right)\left(-a_{3}+j_{3}+a_{2}\right)\left(a_{3}-j_{3}+a_{2}\right)\left(a_{3}+j_{3}-a_{2}+1\right)\right]^{1 / 2},
\end{aligned}
$$

where we have used the fact that $(-1)^{2 j_{1}+2 j_{2}+2 a_{2}}(-1)^{2 j_{3}+2 a_{3}+2 a_{2}}=1$ due to the triangle condition for $\left(j_{1}, j_{2}, a_{2}\right)$ and $\left(a_{2}, j_{3}, a_{3}\right)$.

(II) $I=1, J=2, K=4$

In this case, the general matrix element formula (4.27) reduces to

$$
\begin{aligned}
& \left\langle\vec{a}^{\prime}\left|\hat{q}_{124}\right| \vec{a}\right\rangle=\frac{1}{2}(-1)^{j_{1}+j_{2}-j_{3}+j_{4}+a_{4}} X\left(j_{2}, j_{4}\right)^{\frac{1}{2}} \sqrt{\left(2 a_{2}^{\prime}+1\right)\left(2 a_{2}+1\right)} \\
& \times \sqrt{\left(2 a_{3}^{\prime}+1\right)\left(2 a_{3}+1\right)}\left\{\begin{array}{ccc}
j_{1} & j_{2} & a_{2} \\
1 & a_{2}^{\prime} & j_{2}
\end{array}\right\}\left\{\begin{array}{ccc}
j_{3} & a_{2}^{\prime} & a_{3}^{\prime} \\
1 & a_{3} & a_{2}
\end{array}\right\}\left\{\begin{array}{ccc}
a_{4} & j_{4} & a_{3} \\
1 & a_{3}^{\prime} & j_{4}
\end{array}\right\} \\
& \times\left[a_{2}^{\prime}\left(a_{2}^{\prime}+1\right)-a_{2}\left(a_{2}+1\right)\right] \times\left\{\begin{array}{cc}
\prod_{t=4}^{n-1} \delta_{a_{t}^{\prime}, a_{t}}, & \text { for } n>4 \\
1, & \text { for } n=4
\end{array} .\right.
\end{aligned}
$$

(III) $I=1, J=3, K=4$

In this case, the general matrix element formula (4.27) reduces to

$$
\begin{aligned}
\left\langle\vec{a}^{\prime}\left|\hat{q}_{134}\right| \vec{a}\right\rangle= & -\frac{1}{4}(-1)^{j_{1}+j_{2}+j_{4}+a_{4}} X\left(j_{1}, j_{3}\right)^{\frac{1}{2}} X\left(j_{3}, j_{4}\right)^{\frac{1}{2}} \sqrt{\left(2 a_{2}^{\prime}+1\right)\left(2 a_{2}+1\right)} \sqrt{\left(2 a_{3}^{\prime}+1\right)\left(2 a_{3}+1\right)} \\
& \times\left\{\begin{array}{ccc}
j_{2} & j_{1} & a_{2}^{\prime} \\
1 & a_{2} & j_{1}
\end{array}\right\}\left\{\begin{array}{ccc}
a_{4} & j_{4} & a_{3} \\
1 & a_{3}^{\prime} & j_{4}
\end{array}\right\}\left[(-1)^{a_{2}^{\prime}+a_{3}^{\prime}}\left\{\begin{array}{ccc}
a_{3} & j_{3} & a_{2} \\
1 & a_{2}^{\prime} & j_{3}
\end{array}\right\}\left\{\begin{array}{ccc}
a_{2}^{\prime} & j_{3} & a_{3} \\
1 & a_{3}^{\prime} & j_{3}
\end{array}\right\}-(-1)^{a_{2}+a_{3}}\left\{\begin{array}{ccc}
a_{3}^{\prime} & j_{3} & a_{2} \\
1 & a_{2}^{\prime} & j_{3}
\end{array}\right\}\left\{\begin{array}{ccc}
a_{2} & j_{3} & a_{3} \\
1 & a_{3}^{\prime} & j_{3}
\end{array}\right\}\right] \\
& \times\left\{\begin{array}{cc}
\prod_{t=4}^{n-1} \delta_{a_{t}^{\prime}, a_{t}}, & \text { for } n>4 \\
1, & \text { for } n=4
\end{array} .\right.
\end{aligned}
$$

(IV) $I=2, J=3, K=4$

In this case, we have $a_{I-1}=a_{1}=j_{1}$. Then the general matrix element formula (4.27) reduces to

$$
\begin{aligned}
\left\langle\vec{a}^{\prime}\left|\hat{q}_{234}\right| \vec{a}\right\rangle= & -\frac{1}{4}(-1)^{j_{1}+j_{2}+j_{4}+a_{4}}(-1)^{a_{2}-a_{2}^{\prime} X\left(j_{2}, j_{3}\right)^{\frac{1}{2}} X\left(j_{3}, j_{4}\right)^{\frac{1}{2}}} \\
& \times \sqrt{\left(2 a_{2}^{\prime}+1\right)\left(2 a_{2}+1\right)} \sqrt{\left(2 a_{3}^{\prime}+1\right)\left(2 a_{3}+1\right)}\left\{\begin{array}{ccc}
j_{1} & j_{2} & a_{2} \\
1 & a_{2}^{\prime} & j_{2}
\end{array}\right\}\left\{\begin{array}{ccc}
a_{4} & j_{4} & a_{3} \\
1 & a_{3}^{\prime} & j_{4}
\end{array}\right\} \\
& \times\left[(-1)^{a_{2}^{\prime}+a_{3}^{\prime}}\left\{\begin{array}{ccc}
a_{3} & j_{3} & a_{2} \\
1 & a_{2}^{\prime} & j_{3}
\end{array}\right\}\left\{\begin{array}{ccc}
a_{2}^{\prime} & j_{3} & a_{3} \\
1 & a_{3}^{\prime} & j_{3}
\end{array}\right\}-(-1)^{a_{2}+a_{3}}\left\{\begin{array}{ccc}
a_{3}^{\prime} & j_{3} & a_{2} \\
1 & a_{2}^{\prime} & j_{3}
\end{array}\right\}\left\{\begin{array}{ccc}
a_{2} & j_{3} & a_{3} \\
1 & a_{3}^{\prime} & j_{3}
\end{array}\right\}\right]
\end{aligned}
$$




$$
\times\left\{\begin{array}{cc}
\prod_{t=4}^{n-1} \delta_{a_{t}^{\prime}, a_{t}}, & \text { for } n>4 \\
1, & \text { for } n=4
\end{array}=(-1)^{a_{2}-a_{2}^{\prime}} \frac{X\left(j_{2}, j_{3}\right)^{\frac{1}{2}}}{X\left(j_{1}, j_{3}\right)^{\frac{1}{2}}} \frac{\left\{\begin{array}{ccc}
j_{1} & j_{2} & a_{2} \\
1 & a_{2}^{\prime} & j_{2}
\end{array}\right\}}{\left\{\begin{array}{ccc}
j_{2} & j_{1} & a_{2}^{\prime} \\
1 & a_{2} & j_{1}
\end{array}\right\}}\left\langle\vec{a}^{\prime}\left|\hat{q}_{134}\right| \vec{a}\right\rangle\right.
$$

\section{The Hamiltonian constraint operator}

In Hamiltonian formulation of GR, the dynamical information of GR is encoded in the Hamiltonian constraint. In canonical LQG, while the kinematical structure has been successfully established, the implementation of the Hamiltonian constraint in the quantum theory is still in exploration. Hence the quantum dynamics of LQG is still an open problem up to now. After the pioneering work of Rovelli and Smolin [48], Thiemann first constructed a well-defined Hamiltonian constraint operator in $\mathcal{H}_{\text {kin }}[4,16]$. This operator is anomaly-free in some sense $[4,16]$. The technique to quantize the Hamiltonian constraint in LQG has also been applied to the coupling of gravity with matter [17], high-dimensional GR [49], the scalar-tensor theories of gravity [50,51], and the symmetry-reduced models of LQG [25,52-55]. Moreover, to understand the relation between the canonical and covariant quantum dynamics, one also needs to calculate the matrix elements of the Hamiltonian constraint on given quantum states. In this section, we first recall the construction of Thiemann's Hamiltonian constraint operator, and then derive the action of the Euclidean Hamiltonian constraint operator on a spin-network function over trivalent vertices.

\subsection{Quantization of the Hamiltonian constraint}

The classical Hamiltonian constraint of pure gravity in the connection formulation of GR is given by

$H_{\mathrm{gr}}(N)=\frac{1}{2 \kappa} \int_{\Sigma} \mathrm{d}^{3} x N \frac{\tilde{E}_{i}^{a} \tilde{E}_{j}^{b}}{\sqrt{\operatorname{det}(q)}}\left[\epsilon_{i j k} F_{a b}^{k}-2\left(1+\beta^{2}\right) K_{[a}^{i} K_{b]}^{j}\right]=: H^{E}(N)-2\left(1+\beta^{2}\right) T(N)$,

where $F_{a b}^{k}$ is the curvature of $S U(2)$ connection $A_{c}^{i}$, and $K_{(a}^{i} e_{b) i}$ is the extrinsic curvature of a spatial hypersurface $\Sigma$ in a spacetime. The function $H^{E}(N)$ is called the Euclidean Hamiltonian constraint. In the following, we focus on the regularization of $H^{E}(N)$. Let us triangulate $\Sigma$ into tetrahedra $\Delta$ so that the above integral becomes a sum of integrals over $\Delta$, i.e., $\int_{\Sigma}=\sum_{\Delta} \int_{\Delta}$. We denote the triangulation of $\Sigma$ by $T(\epsilon)$. The small parameter $\epsilon$ indicates the "length" of the edges of $\Delta$. For each $\Delta$, we single out one of its vertices and call it the base-point $v(\Delta)$ of $\Delta$ and denote its three edges outgoing from $v(\Delta)$ by $s_{I}(\Delta), I=1,2,3$. Taking the limit $\epsilon \rightarrow 0$ corresponds to shrinking $\Delta$ to $v(\Delta)$. Let $\alpha_{I J}(\Delta):=s_{I}(\Delta) \circ a_{I J} \circ s_{J}^{-1}(\Delta)$ be the loop based at $v(\Delta)$, where $a_{I J}$ is the edge of $\Delta$ from the endpoint of $s_{I}(\Delta)$ to the endpoint of $s_{J}(\Delta)$. Then the Euclidean Hamiltonian constraint can be written in the form $[4,16]$

$$
\begin{aligned}
H^{E}(N) & =\frac{1}{2 \kappa} \int_{\Sigma} \mathrm{d}^{3} x N \epsilon_{i j k} \frac{\tilde{E}_{i}^{a} \tilde{E}_{j}^{b}}{\sqrt{\operatorname{det}(q)}} F_{a b}^{k}=-\frac{2}{\kappa^{2} \beta} \sum_{\Delta \in T(\epsilon)} \int_{\Delta} \mathrm{d}^{3} x \bar{N} \tilde{\epsilon}^{a b c} \operatorname{tr}\left(F_{a b}\left\{A_{c}, V\right\}\right) \\
& =\lim _{\epsilon \rightarrow 0} \frac{2}{3 \kappa^{2} \beta} \sum_{\Delta \in T(\epsilon)} \bar{N}(v(\Delta)) \epsilon^{I J K} \operatorname{tr}\left(h_{\alpha_{I J}(\Delta)} h_{s_{K}(\Delta)}\left\{h_{S_{K}(\Delta)}^{-1}, V\right\}\right)=: \lim _{\epsilon \rightarrow 0} H_{T(\epsilon)}^{E}(N),
\end{aligned}
$$

where $\bar{N}:=\operatorname{sgn}\left(\operatorname{det}\left(e_{d}^{l}\right)\right) N, A_{c}:=A_{c}^{k} \tau_{k}, V$ denotes the volume function of $\Sigma$, and in the second step we have used the identities

$\epsilon_{i j k} \frac{\tilde{E}_{i}^{a} \tilde{E}_{j}^{b}}{\sqrt{\operatorname{det}(q)}}=\operatorname{sgn}\left(\operatorname{det}\left(e_{d}^{l}\right)\right) \tilde{\epsilon}^{a b c} e_{c}^{k}=\operatorname{sgn}\left(\operatorname{det}\left(e_{d}^{l}\right)\right) \tilde{\epsilon}^{a b c} \frac{2}{\kappa \beta}\left\{A_{c}^{m}, V\right\} \delta_{k m}, \quad \operatorname{tr}\left(\tau_{k} \tau_{m}\right)=-\frac{1}{2} \delta_{k m}$.

To simplify the notations, we will drop the bar over $N$. Replacing $V$ by $\hat{V}$, holonomies by holonomy operators (since the holonomy operator acts as a multiplication operator, we also omit the hat for simplification of notation), and the Poisson 
bracket by $1 /(i \hbar)$ times the commutator, the regulated Euclidean Hamiltonian constraint operator reads

$$
\begin{aligned}
\hat{H}_{T(\epsilon)}^{E}(N) & =\frac{2}{3 i \hbar \kappa^{2} \beta} \sum_{\Delta \in T(\epsilon)} N(v(\Delta)) \epsilon^{I J K} \operatorname{tr}\left(h_{\alpha_{I J}(\Delta)} h_{s_{K}(\Delta)}\left[h_{s_{K}(\Delta)}^{-1}, \hat{V}\right]\right) \\
& =-\frac{2}{3 i \hbar \kappa^{2} \beta} \sum_{\Delta \in T(\epsilon)} N(v(\Delta)) \epsilon^{I J K} \operatorname{tr}\left(h_{\alpha_{I J}(\Delta)} h_{s_{K}(\Delta)} \hat{V} h_{s_{K}(\Delta)}^{-1}\right) \\
& =:-\frac{2}{3 i \hbar \kappa^{2} \beta} \sum_{\Delta \in T(\epsilon)} N(v(\Delta)) \hat{H}_{\Delta}^{E} .
\end{aligned}
$$

It is clear that the operator (5.4) depends on the triangulation $T(\epsilon)$. It turns out that the non-trivial action of $\hat{H}_{\Delta}^{E}$ on a cylindrical function $f_{\gamma}$ corresponds to the case $v(\Delta) \cap \gamma \neq \emptyset$. Thus one can triangulate $\Sigma$ adapted to $\gamma$ [16]. We denote the triangulation adapted to $\gamma$ by $T(\gamma)$. Then the action of the regularized Euclidean Hamiltonian constraint operator (5.4) on $f_{\gamma}$ reduces to [16]

$\hat{H}_{\gamma}^{E}(N) \cdot f_{\gamma}:=\hat{H}_{T(\gamma)}^{E}(N) \cdot f_{\gamma}=-\frac{2}{3 i \hbar \kappa^{2} \beta} \sum_{v \in V(\gamma)} N(v) \frac{8}{E(v)} \sum_{v(\Delta)=v} \hat{H}_{\Delta}^{E} \cdot f_{\gamma}=: \sum_{v \in V(\gamma)} N_{v}^{\prime} \hat{H}_{v}^{E} \cdot f_{\gamma}$,

where $E(v)$ is the number of non-planar triples of edges of $\gamma$ or $\gamma^{\prime}$ at $v, N_{v}^{\prime}:=-\frac{16}{3 i \hbar \kappa^{2} \beta} \frac{N(v)}{E(v)}$, and

$\hat{H}_{v}^{E}:=\sum_{v(\Delta)=v} \epsilon^{I J K} \operatorname{tr}\left(h_{\alpha_{I J}(\Delta)} h_{s_{K}(\Delta)} \hat{V} h_{s_{K}(\Delta)}^{-1}\right)$

The limit $\epsilon \rightarrow 0$ can be taken in a natural operator topology $[4,16]$. The label $T$ for the triangulation $T(\gamma)$ can be dropped since the final limit operator is independent of $\epsilon$.

5.2 The action of $\hat{H}_{\gamma}^{E}(N)$ on a trivalent non-planar vertex

The action of the Hamiltonian constraint (5.5) is local in the sense that it is a sum over independent vertices. Therefore, we can concentrate on its action on a single vertex. For a spin-network state $T_{\gamma, \vec{j}, \vec{i}}(A)$ on a graph $\gamma$, we consider a trivalent non-planar vertex $v \in V(\gamma)$ at which three edges $e_{1}, e_{2}, e_{3}$ incident. The terms in $T_{\gamma, \vec{j}, \vec{i}}(A)$ directly associated to $v$ can be represented by

$T_{\gamma, \vec{j}, \vec{i}}^{v}(A):=\left(i_{v}\right)_{m_{1} m_{2} m_{3}}\left[\pi_{j_{1}}\left(h_{e_{1}}\right)\right]_{n_{1}}^{m_{1}}\left[\pi_{j_{2}}\left(h_{e_{2}}\right)\right]_{n_{2}}^{m_{2}}\left[\pi_{j_{3}}\left(h_{e_{3}}\right)\right]_{n_{3}}^{m_{3}}$,

where $\left(i_{v}\right)_{m_{1} m_{2} m_{3}} \equiv\left(i_{j_{1}, j_{2}, j_{3}}^{J=0 ; \vec{a} \equiv\left\{a_{2}=j_{3}\right\}}\right)_{m_{1} m_{2} m_{3}}^{M=0}$ denotes the intertwiner associated to $v$. For the trivalent non-planar vertex $v$ under consideration, the summation in the expression of $\hat{H}_{v}^{E}$ in (5.6) is over only one tetrahedron $\Delta$ adapted to $\gamma$ at $v$. We will omit the notation $\Delta$. Then the action of $\hat{H}_{v}^{E}$ on $T_{\gamma, \vec{j}, \vec{i}}^{v}(A)$ can be explicitly written as

$$
\begin{aligned}
\hat{H}_{v}^{E} \cdot T_{\gamma, \vec{j}, \vec{i}}^{v}(A)= & \left.\epsilon^{I J K} \operatorname{tr}\left(h_{\alpha_{I J}} h_{s_{K}} \hat{V} h_{s_{K}}^{-1}\right) \cdot T_{\gamma, \vec{j}, \vec{i}}^{v}(A)=\epsilon^{I J K} h_{\alpha_{I J}}\right]_{B}^{A}\left[h_{s_{K}}\right]_{C}^{B} \hat{V}\left[h_{s_{K}}^{-1}\right]_{A}^{C} \cdot T_{\gamma, \vec{j}, \vec{i}}^{v}(A) \\
= & {\left[h_{\alpha_{23}}-h_{\alpha_{32}}\right]_{B}^{A}\left[h_{s_{1}}\right]_{C}^{B} \hat{V}\left[h_{s_{1}}^{-1}\right]_{A}^{C} \cdot T_{\gamma, \vec{j}, \vec{i}}^{v}(A)+\left[h_{\alpha_{31}}-h_{\alpha_{13}}\right]_{B}^{A}\left[h_{s_{2}}\right]_{C}^{B} \hat{V}_{\left.h_{s_{2}}\right]}^{-1}{ }_{A} \cdot T_{\gamma, \vec{j}, \vec{i}}^{v}(A) } \\
& +\left[h_{\alpha_{12}}-h_{\left.\alpha_{21}\right]_{B}}^{A}\left[h_{s_{3}}\right]_{C}^{B} \hat{V}\left[h_{s_{3}}^{-1}\right]_{A}^{C} \cdot T_{\gamma, \vec{j}, \vec{i}}^{v}(A) \equiv\left(\hat{H}_{v, s_{2} s_{3} s_{1}}^{E}+\hat{H}_{v, s_{3} s_{1} s_{2}}^{E}+\hat{H}_{v, s_{1} s_{2} s_{3}}^{E}\right) \cdot T_{\gamma, \vec{j}, \vec{i}}^{v}(A),\right.
\end{aligned}
$$

where $[h]_{B}^{A} \equiv\left[\pi_{1 / 2}(h)\right]_{B}^{A}$. Note that applying $\hat{H}_{v}^{E}$ to $T_{\gamma, \vec{j}, \vec{i}}^{v}(A)$ involves the actions of the holonomy and the volume operators.

The intertwiner associated to $v$ in (5.7) is represented in graphical formula as

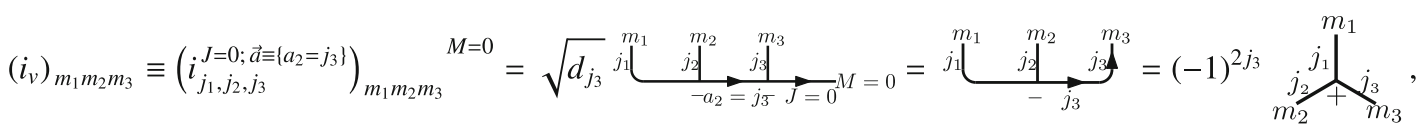


where $d_{j_{3}} \equiv 2 j_{3}+1$, and in the last two steps we have used Eqs. (A.47) and (A.42). The part of $\gamma$ associated at $v$ and the spin-network state $T_{\gamma, \vec{j}, \vec{i}}^{v}(A)$ in Eq. (5.7) can be represented, respectively, by
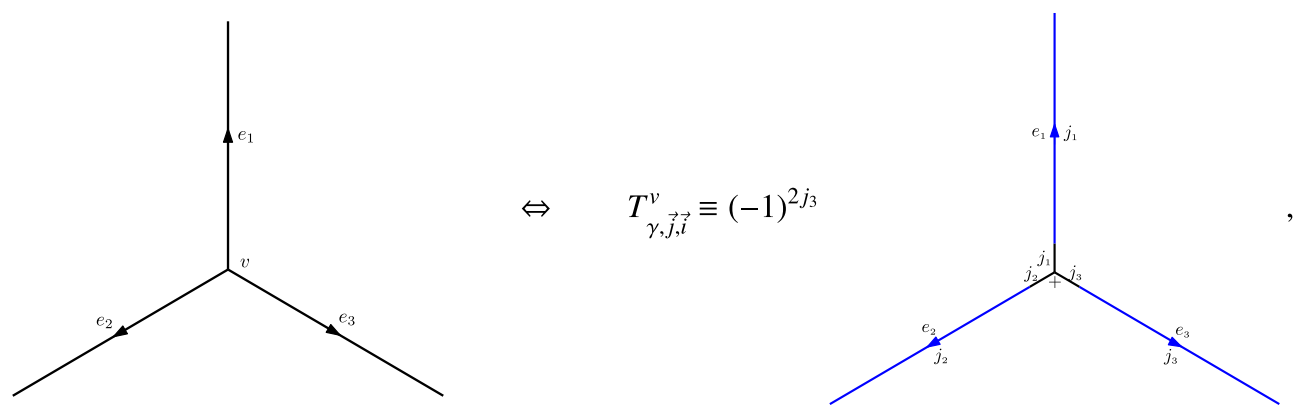

where we have omitted the remaining notations $n_{1}, n_{2}, n_{3}$ in the algebraic form (5.7). By introducing three pseudo-vertices $\tilde{v}_{I}, I=1,2,3$, we subdivide $e_{I}$ into two parts $s_{I}$ and $l_{I}$ such that $e_{I}=s_{I} \circ l_{I}$ and $s_{I}=s_{I}(\Delta)$ matching the triangulation $T(\gamma)$. Then $T_{\gamma, \vec{j}, \vec{i}}^{v}(A)$ in Eq. (5.7) becomes

$$
\begin{aligned}
T_{\gamma, \vec{j}, \vec{i}}^{v}(A)= & \left(i_{v}\right)_{m_{1} m_{2} m_{3}}\left[\pi_{j_{1}}\left(h_{s_{1}}\right)\right]_{{ }_{l_{1}}}^{m_{1}} \delta_{k_{1}}^{l_{1}}\left[\pi_{j_{1}}\left(h_{l_{1}}\right)\right]_{{ }_{n_{1}}}^{k_{1}}\left[\pi_{j_{2}}\left(h_{s_{2}}\right)\right]^{m_{2}}{ }_{l_{2}} \delta_{k_{2}}^{l_{2}}\left[\pi_{j_{2}}\left(h_{l_{2}}\right)\right]_{n_{2}}^{k_{2}}\left[\pi_{j_{3}}\left(h_{s_{3}}\right)\right]_{l_{3}}^{m_{3}} \delta_{k_{3}}^{l_{3}}\left[\pi_{j_{3}}\left(h_{l_{3}}\right)\right]_{n_{3}}^{k_{3}} \\
= & \left(i_{v}\right)_{m_{1} m_{2} m_{3}}\left[\pi_{j_{1}}\left(h_{s_{1}}\right)\right]^{m_{1}}{ }_{l_{1}}\left[\pi_{j_{2}}\left(h_{s_{2}}\right)\right]^{m_{2}}{ }_{l_{2}}\left[\pi_{j_{3}}\left(h_{s_{3}}\right)\right]^{m_{3}}{ }_{l_{3}}\left(i_{\tilde{v}_{1}}\right)_{k_{1}} l_{1} \\
& \times\left(i_{\tilde{v}_{2}}\right)_{k_{2}}{ }_{2}\left(i_{\tilde{v}_{3}}\right)_{k_{3}}{ }^{l_{3}}\left[\pi_{j_{1}}\left(h_{l_{1}}\right)\right]_{n_{1}}^{k_{1}}\left[\pi_{j_{2}}\left(h_{l_{2}}\right)\right]_{{ }_{2}}^{k_{2}}\left[\pi_{j_{3}}\left(h_{l_{3}}\right)\right]^{k_{3}}{ }_{n_{3}},
\end{aligned}
$$

where $\left(i_{\tilde{v}_{I}}\right)_{k_{I}}^{l_{I}}=\delta_{k_{I}}^{l_{I}}$ are the intertwiners associated to $\tilde{v}_{I}$. Hence the original graph and the corresponding spin-network state in (5.10) become

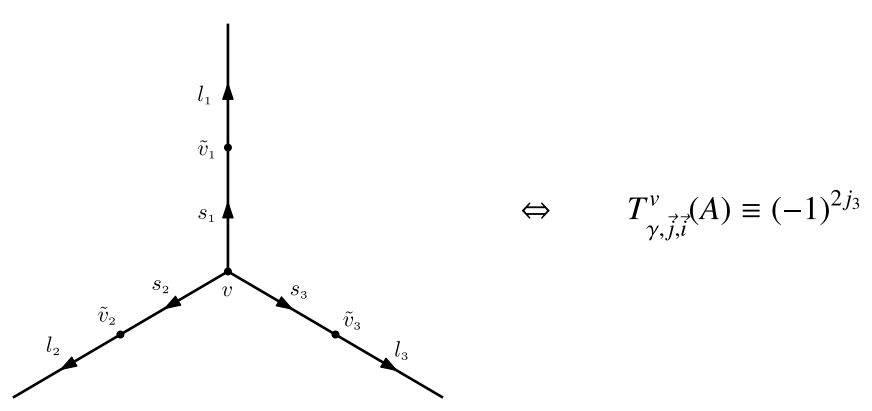

We can also single out the part of $T_{\gamma, \vec{j}, \vec{i}}^{v}(A)$ which only involves the holonomies $h_{s_{I}}$ and denote it by $T_{\gamma, \vec{j}, \vec{i}}^{v, s}(A)$ (the notation $s$ denotes the segments $s_{I}$ ), i.e.,

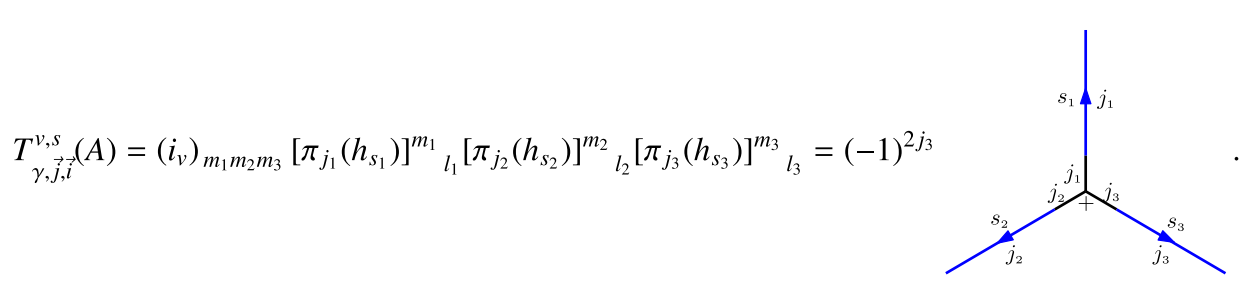


Now let us to calculate the action of the first term in (5.8) on $T_{\gamma, \vec{j}, \vec{i}}^{v, s}(A)$ via the graphical method. The action of $\left[h_{s_{1}}\right]_{C}^{B} \hat{V}\left[h_{s_{1}}^{-1}\right]_{A}^{C}$ on $T_{\gamma, \vec{j}, \vec{i}}^{v, s}(A)$ can be represented by

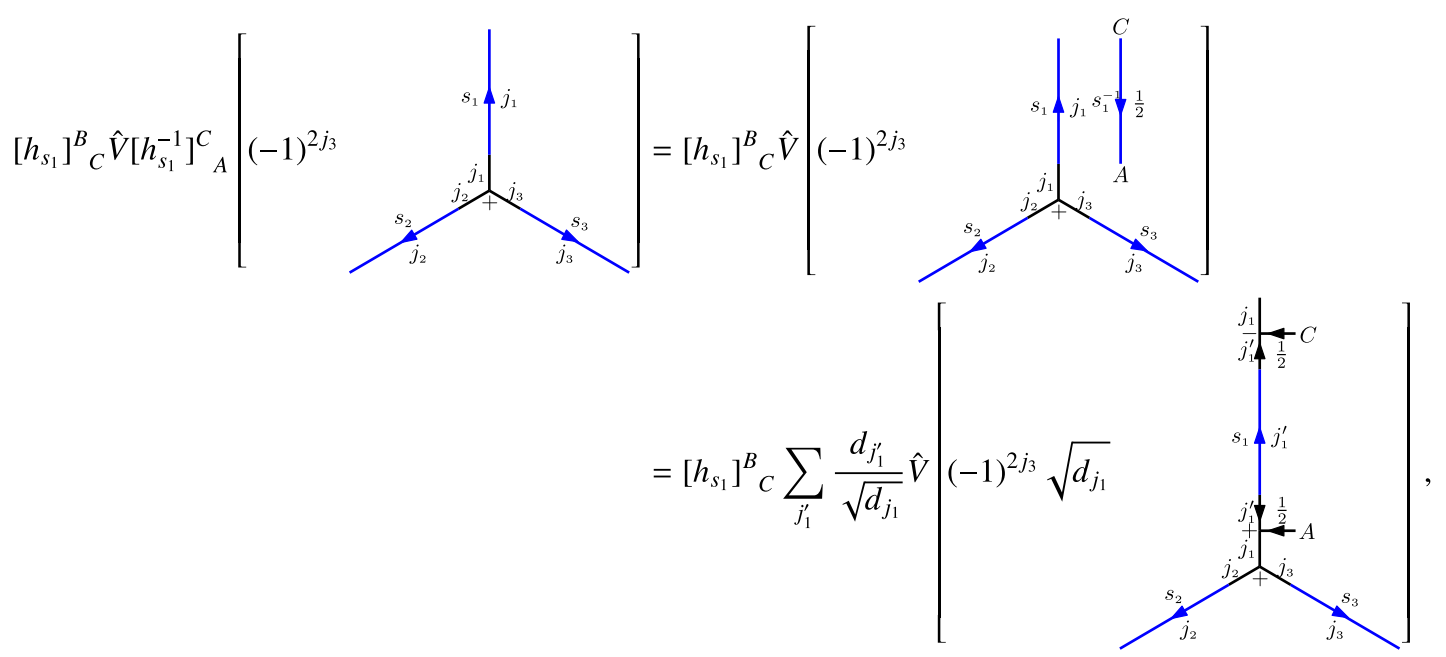

where $j_{1}^{\prime}=j_{1} \pm \frac{1}{2}$, and we have adjusted the coefficients such that the intertwiner $i_{v}^{\prime}$ at $v$ is normalized as

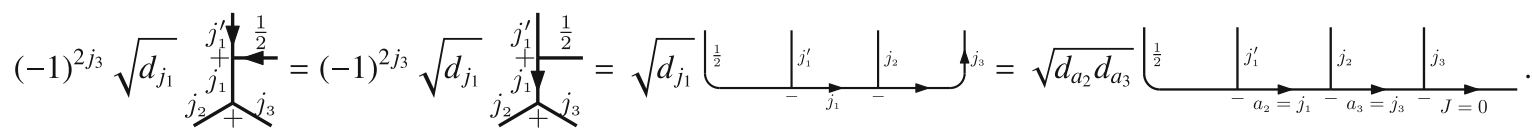

Recall that the volume operator (4.5) vanishes at coplanar vertices. Hence it has non-trivial action only at $v$, not $\tilde{v}_{I}$. Its action in (5.14) reads

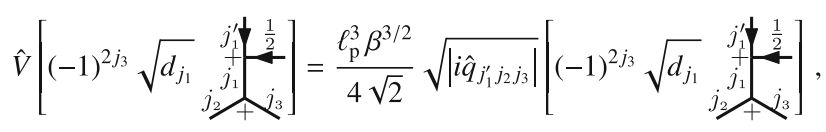

where the operator $\hat{q}_{j_{1}^{\prime} j_{2} j_{3}}$ corresponds to the edges $s_{1}, s_{2}, s_{3}$ with spins $j_{1}^{\prime}, j_{2}, j_{3}$, respectively. Notice that $\hat{q}_{I J K}$ changes only the intermediate momenta $a_{I}, \ldots, a_{K-1}$ between $j_{I}$ and $j_{K}$ of the intertwiner. In our case, the operator $\hat{q}_{j_{1}^{\prime} j_{2} j_{3}}$ and hence $\hat{V}$ change $a_{2}=j_{1}, a_{3}=j_{3}$ into

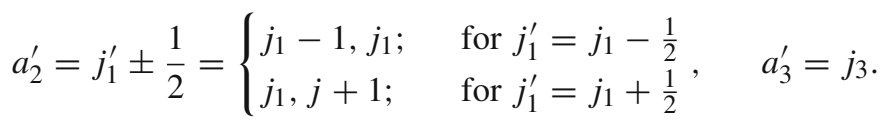

For given values of the four spins $\frac{1}{2}, j_{1}^{\prime}, j_{2}, j_{3}$, there are two allowed combinations of intermediate momenta (5.17). Hence the corresponding intertwiner space associated to $v$ is of dimension 2. Furthermore, the volume operator is automatically diagonal on the 2-dimensional intertwiner space. This fact was pointed out in [5,21], which will also be presented in Appendix C. Hence we have 


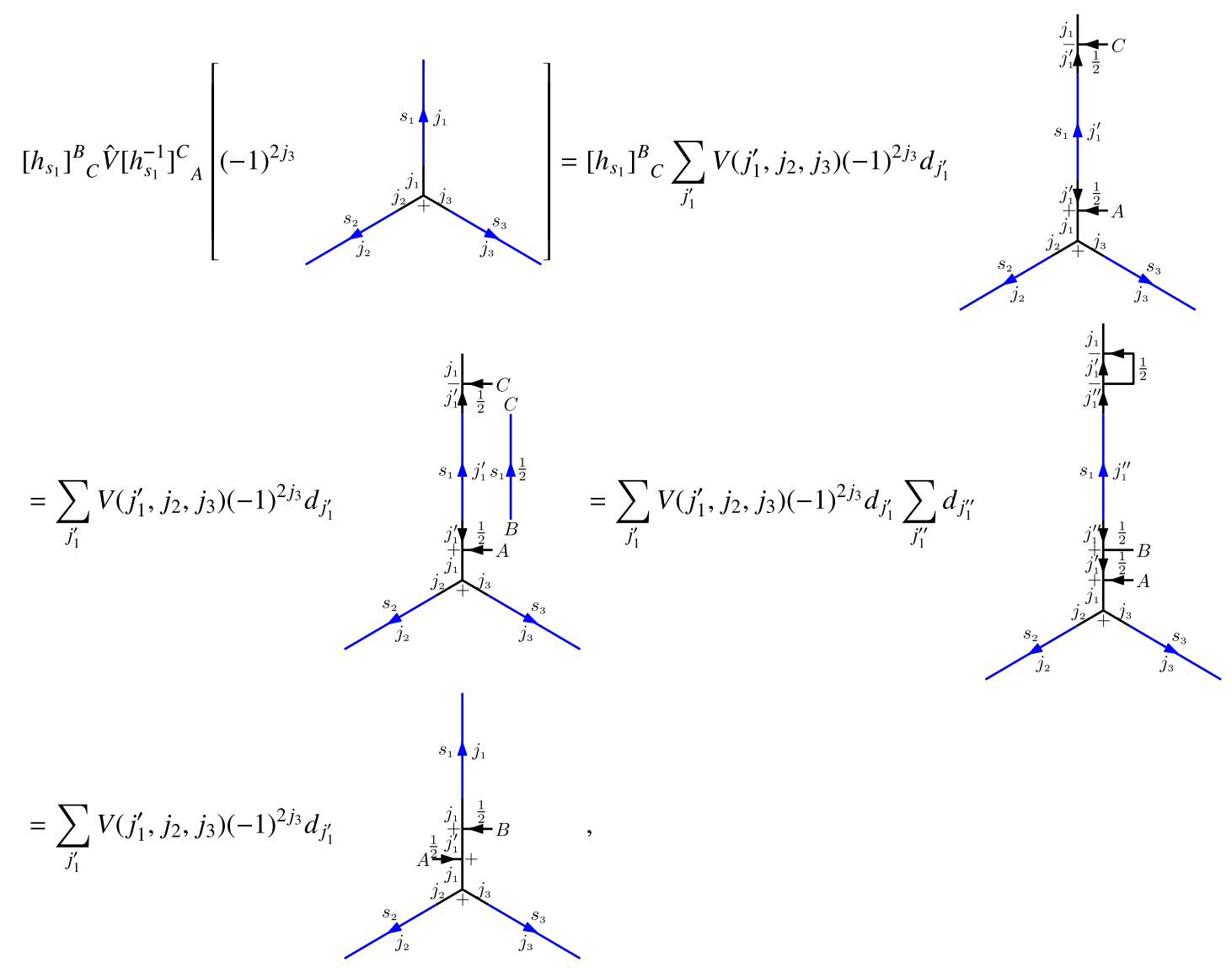

where

$$
\begin{aligned}
V\left(j_{1}^{\prime}, j_{2}, j_{3}\right) \equiv & V\left(1 / 2, j_{1}^{\prime}, j_{2}, j_{3} ; a_{2}=j_{1}^{\prime}+1 / 2, a_{3}=j_{3}\right) \equiv \frac{\ell_{\mathrm{p}}^{3} \beta^{3 / 2}}{4 \sqrt{2}}\left[\left(j_{1}^{\prime}+j_{2}+j_{3}+\frac{3}{2}\right)\left(j_{1}^{\prime}+j_{2}-j_{3}+\frac{1}{2}\right)\right. \\
& \left.\times\left(j_{1}^{\prime}-j_{2}+j_{3}+\frac{1}{2}\right)\left(-j_{1}^{\prime}+j_{2}+j_{3}+\frac{1}{2}\right)\right]^{\frac{1}{4}} .
\end{aligned}
$$

In the fourth step we have changed the orientation of two arrows with spin $j_{1}^{\prime}$ by the rule (A.43), and then used the rule (A.44) to remove three arrows with the same orientation joint with a $3 j$-symbol, and we also used the rule (A.45) to remove a loop. Thus the action of $\hat{H}_{v, s_{2} s_{3} s_{1}}^{E}=\left[h_{\alpha_{23}}-h_{\alpha_{32}}\right]_{B}^{A}\left[h_{s_{1}}\right]_{C}^{B} \hat{V}\left[h_{s_{1}}^{-1}\right]^{C}{ }_{A}$ on $T_{\gamma, \vec{j}, \vec{i}}^{v, s}(A)$ is given by 


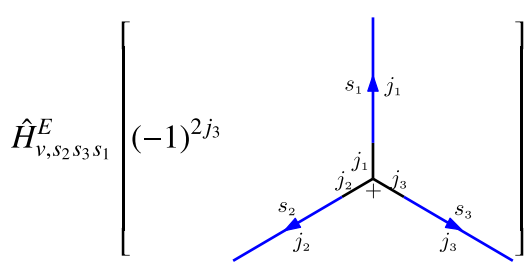
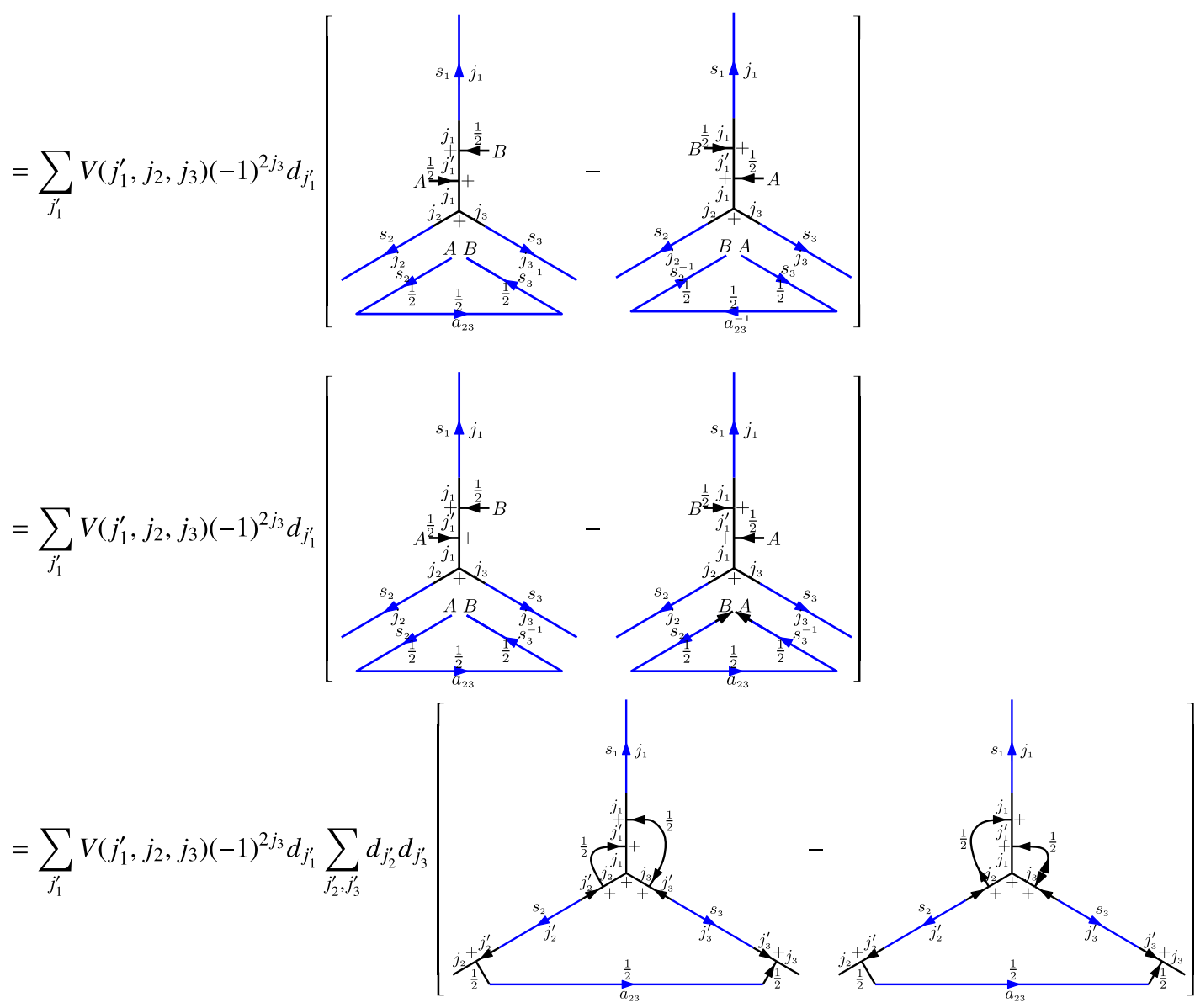

$=\sum_{j_{1}^{\prime}, j_{2}^{\prime}, j_{3}^{\prime}} V\left(j_{1}^{\prime}, j_{2}, j_{3}\right) d_{j_{1}^{\prime}} d_{j_{2}^{\prime}} d_{j_{3}^{\prime}}$

$\times\left[-(-1)^{j_{1}+j_{1}^{\prime}+\frac{1}{2}}(-1)^{j_{3}+j_{3}^{\prime}+\frac{1}{2}}\left\{\begin{array}{lll}j_{1} & \frac{1}{2} & j_{1}^{\prime} \\ j_{2}^{\prime} & j_{3} & j_{2}\end{array}\right\}\left\{\begin{array}{lll}j_{1} & \frac{1}{2} & j_{1}^{\prime} \\ j_{3} & j_{2}^{\prime} & j_{3}^{\prime}\end{array}\right\}-(-1)^{j_{1}-j_{1}^{\prime}+\frac{1}{2}}(-1)^{j_{2}+j_{2}^{\prime}+\frac{1}{2}}\left\{\begin{array}{lll}j_{1} & \frac{1}{2} & j_{1}^{\prime} \\ j_{2} & j_{3}^{\prime} & j_{2}^{\prime}\end{array}\right\}\left\{\begin{array}{lll}j_{1} & \frac{1}{2} & j_{1}^{\prime} \\ j_{3}^{\prime} & j_{2} & j_{3}\end{array}\right\}\right]$

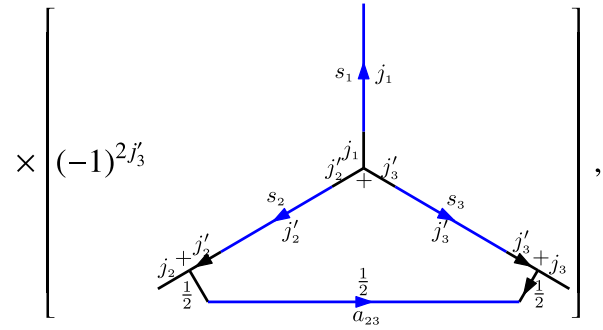


where in the second step we have used (A.57), and in the last step we have used the two identities (see Appendix B.4 for a proof)

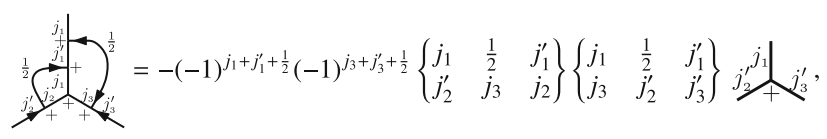

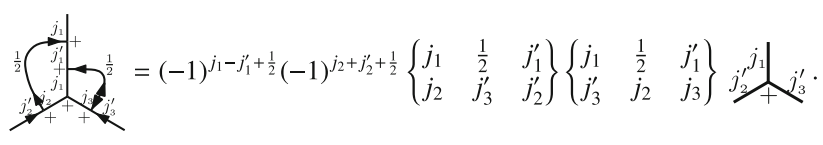

Here Eq. (A.43) was used to flip the orientation of the arrow on the line labeled by $1 / 2$, and the identity $(-1)^{2 j_{3}+1}=(-1)^{2 j_{3}^{\prime}}$ was used. Notice that those three intertwiners associated to $v, \tilde{v}_{2}$ and $\tilde{v}_{3}$ in the last line of Eq. (5.20) take the standard formulas (A.38) and (A.39), i.e.,

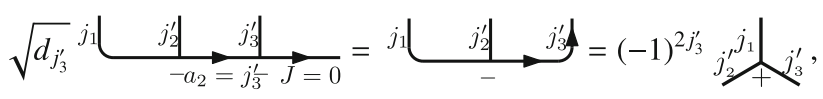

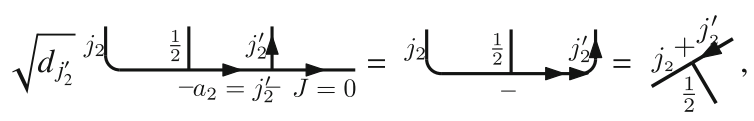

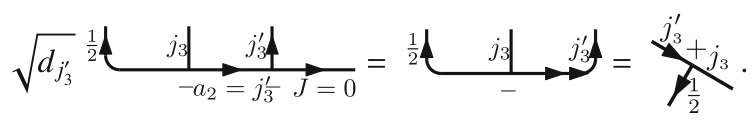

Equation (5.20) enables us to directly write down the results for $(I, J, K) \in\{(2,3,1),(3,1,2),(1,2,3)\}$ as

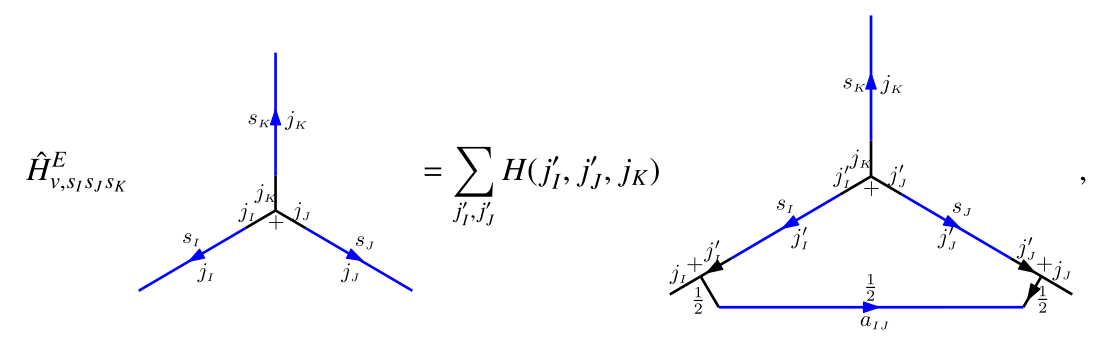

where

$$
\begin{aligned}
H\left(j_{I}^{\prime}, j_{J}^{\prime}, j_{K}\right)= & \sum_{j_{K}^{\prime}} V\left(j_{K}^{\prime}, j_{I}, j_{J}\right) d_{j_{K}^{\prime}} d_{j_{I}^{\prime}} d_{j_{J}^{\prime}}\left[(-1)^{j_{K}+j_{K}^{\prime}+\frac{1}{2}}(-1)^{j_{J}+j_{J}^{\prime}+\frac{1}{2}}\left\{\begin{array}{ccc}
j_{K} & \frac{1}{2} & j_{K}^{\prime} \\
j_{I}^{\prime} & j_{J} & j_{I}
\end{array}\right\}\left\{\begin{array}{ccc}
j_{K} & \frac{1}{2} & j_{K}^{\prime} \\
j_{J} & j_{I}^{\prime} & j_{J}^{\prime}
\end{array}\right\}\right. \\
& \left.+(-1)^{j_{K}-j_{K}^{\prime}+\frac{1}{2}}(-1)^{j_{I}+j_{I}^{\prime}+\frac{1}{2}}\left\{\begin{array}{ccc}
j_{K} & \frac{1}{2} & j_{K}^{\prime} \\
j_{I} & j_{J}^{\prime} & j_{I}^{\prime}
\end{array}\right\}\left\{\begin{array}{ccc}
j_{K} & \frac{1}{2} & j_{K}^{\prime} \\
j_{J}^{\prime} & j_{I} & j_{J}
\end{array}\right\}\right] .
\end{aligned}
$$


Taking account of the identity $(-1)^{2 j_{3}+1}=(-1)^{2 j_{3}^{\prime}}$, the action of $\hat{H}_{v}^{E}$ on $T_{\gamma, \vec{j}, \vec{i}}^{v, s}(A)$ can be explicitly written down,

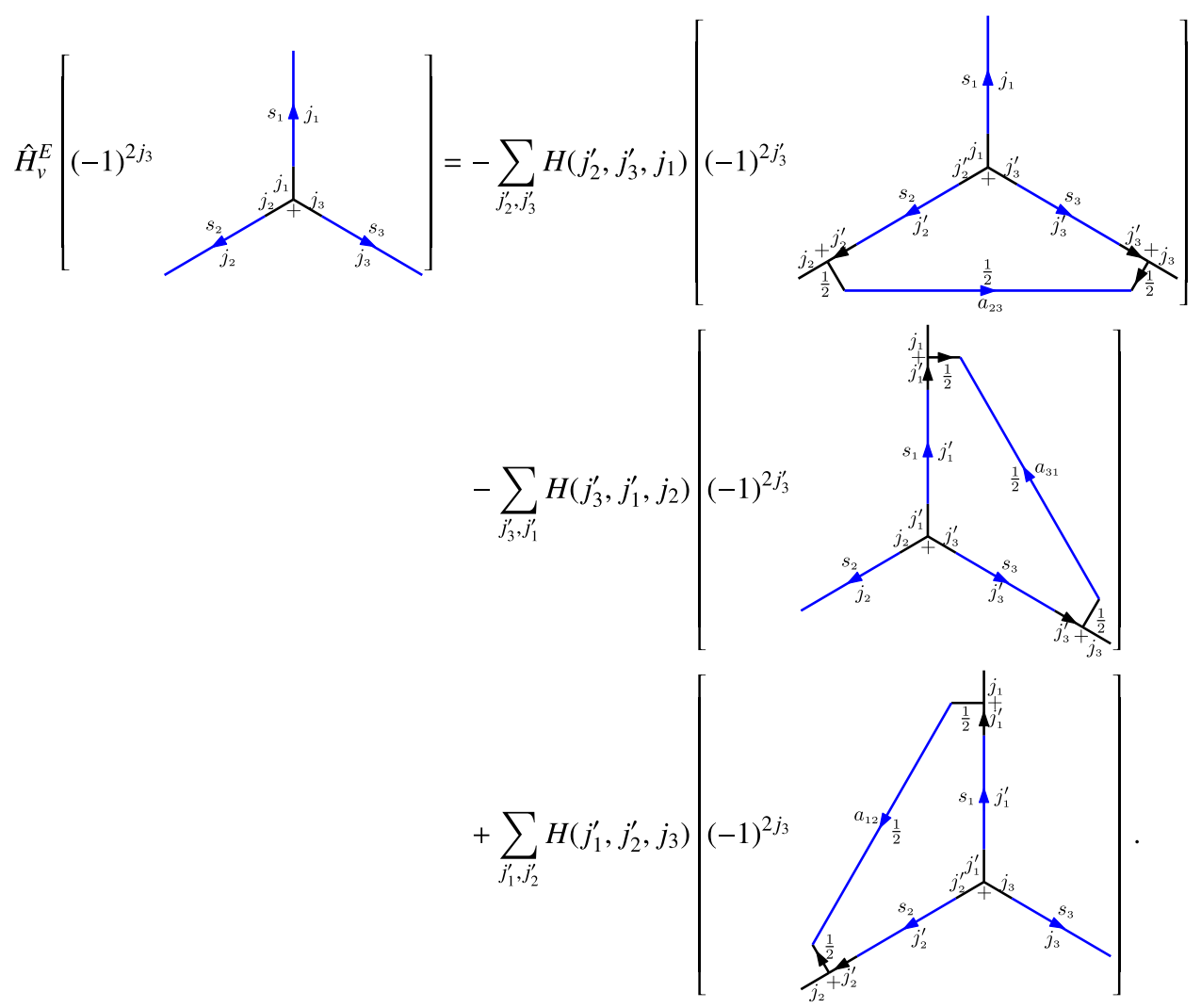

\section{The inverse volume operator}

In this section, we will first review the construction of the inverse volume operator (see [27] for details). Then the action of the inverse volume operator on a gauge-invariant spin-network state $T_{\gamma, \vec{j}, \vec{i}}^{v}(A)$ is calculated at a trivalent non-planar vertex $v$ in the graphical method. Comparing to the algebraic derivation in [27], our graphical calculation is again rather concise.

\subsection{The construction of the inverse volume operator}

The Hamiltonian of a massless scalar field coupling to gravity is given by

$$
H(N)=H_{\mathrm{gr}}(N)+H_{\phi}
$$

where $H_{\mathrm{gr}}(N)$ is the Hamiltonian constraint (5.1) of pure gravity, and $H_{\phi}(N)$ is the Hamiltonian of a massless scalar field $\phi$, which reads

$H_{\phi}(N)=\frac{1}{2} \int_{\Sigma} \mathrm{d}^{3} x N(x)\left[\frac{\pi^{2}}{\sqrt{\operatorname{det}(q)}}+\sqrt{\operatorname{det}(q)} q^{a b}\left(\partial_{a} \phi\right) \partial_{b} \phi\right](x) \equiv \frac{1}{2}\left[H_{\mathrm{kin}, \phi}(N)+H_{\mathrm{der}, \phi}(N)\right]$

where $\pi$ is the momentum conjugate to $\phi$. The Hamiltonian $H(N)$ can be quantized as a well-defined operator on the Hilbert space $\mathcal{H}_{\mathrm{gr}} \otimes \mathcal{H}_{\phi}$ with $\mathcal{H}_{\mathrm{gr}} \equiv \mathcal{H}_{\text {kin }}$ and $\mathcal{H}_{\phi}$ being the kinematical Hilbert space for the scalar field theory [17,56]. Notice that in the isotropic cosmological models the inverse of volume function (or scale factor) appears also in the term $H_{\text {kin, } \phi}(N)$. In order to compare the results between LQC and LQG, we will focus on this term. To do the quantization, the term $H_{\text {kin, } \phi}(N)$ 
can be regularized as [17]

$$
\begin{aligned}
H_{\text {kin }, \phi}(N)= & \int_{\Sigma} \mathrm{d}^{3} x N(x) \frac{\pi^{2}(x)}{\sqrt{\operatorname{det}(q)(x)}}=\lim _{\epsilon \rightarrow 0} \int_{\Sigma} \mathrm{d}^{3} x N(x) \pi(x) \int_{\Sigma} \mathrm{d}^{3} y \pi(y) \int_{\Sigma} \mathrm{d}^{3} u \frac{\operatorname{det}\left(e_{a}^{i}\right)}{\left[\epsilon^{3} \sqrt{\operatorname{det}(q)}\right]^{3 / 2}}(u) \\
& \times \int_{\Sigma} \mathrm{d}^{3} w \frac{\operatorname{det}\left(e_{a}^{i}\right)}{\left[\epsilon^{3} \sqrt{\operatorname{det}(q)}\right]^{3 / 2}}(w) \chi_{\epsilon}(x, y) \chi_{\epsilon}(x, u) \chi_{\epsilon}(x, w) \\
= & \frac{2^{6} \cdot 2^{6}}{3 ! \cdot 3 ! \cdot \kappa^{6} \cdot \beta^{6}} \lim _{\epsilon \rightarrow 0} \int_{\Sigma} \mathrm{d}^{3} x N(x) \pi(x) \int_{\Sigma} \mathrm{d}^{3} y \pi(y) \\
& \times \int_{\Sigma} \mathrm{d}^{3} u \tilde{\epsilon}^{a b c} \epsilon_{i j k}\left\{A_{a}^{i}(u), V(u, \epsilon)^{\frac{1}{2}}\right\}\left\{A_{b}^{j}(u), V(u, \epsilon)^{\frac{1}{2}}\right\}\left\{A_{c}^{k}(u), V(u, \epsilon)^{\frac{1}{2}}\right\} \\
& \times \int_{\Sigma} \mathrm{d}^{3} w \tilde{\epsilon}^{d e f} \epsilon_{l m n}\left\{A_{d}^{l}(w), V(w, \epsilon)^{\frac{1}{2}}\right\}\left\{A_{e}^{m}(w), V(w, \epsilon)^{\frac{1}{2}}\right\}\left\{A_{f}^{n}(w), V(w, \epsilon)^{\frac{1}{2}}\right\} \times \chi_{\epsilon}(x, y) \chi_{\epsilon}(x, u) \chi_{\epsilon}(x, w),
\end{aligned}
$$

where we have inserted $1=\left[\operatorname{det}\left(e_{a}^{i}\right)\right]^{2} /[\sqrt{\operatorname{det}(q)}]^{2}$ in the second step, used $e_{a}^{i}(x)=\frac{2}{\kappa \beta}\left\{A_{a}^{i}(x), V(x, \epsilon)\right\}$, and absorbed $V(x, \epsilon):=\epsilon^{3} \sqrt{\operatorname{det}(q)}(x)$ in the denominator into the Poisson bracket in the last step. Again we introduce a triangulation $T(\gamma)$ of $\Sigma$ adapted to a graph $\gamma$. For a given tetrahedron $\Delta$ and its edge $s_{I}(\Delta)=: s_{I}$, by the identity

$$
\int_{S_{I}(\Delta)} \mathrm{d}^{3} x\left\{A_{a}^{i}(x), V(x, \epsilon)^{\frac{1}{2}}\right\}=2 \operatorname{tr}\left(\tau_{i} h_{I}\left\{h_{I}^{-1}, V(v, \epsilon)^{\frac{1}{2}}\right\}\right)+O\left(\epsilon^{2}\right), \quad h_{I} \equiv h_{S_{I}(\Delta)},
$$

Eq. (6.3) can be reduced to

$$
\begin{aligned}
H_{\text {kin }, \phi}(N)= & \frac{2^{22}}{3^{2} \cdot \kappa^{6} \cdot \beta^{6}} \lim _{\epsilon \rightarrow 0} \int_{\Sigma} \mathrm{d}^{3} x N(x) \pi(x) \int_{\Sigma} \mathrm{d}^{3} y \pi(y) \sum_{v, v^{\prime} \in V(\gamma)} \frac{1}{E(v) E\left(v^{\prime}\right)} \sum_{\substack{s_{I} \cap s_{J} \cap s_{K}=v \\
s_{L} \cap s_{M} \cap s_{N}=v^{\prime}}} \epsilon^{I J K} \epsilon^{L M N} \epsilon^{i j k} \epsilon^{l m n} \\
& \times \operatorname{tr}\left(\tau_{i} h_{I}\left\{h_{I}^{-1}, V(v, \epsilon)^{\frac{1}{2}}\right\}\right) \operatorname{tr}\left(\tau_{l} h_{L}\left\{h_{L}^{-1}, V\left(v^{\prime}, \epsilon\right)^{\frac{1}{2}}\right\}\right) \operatorname{tr}\left(\tau_{j} h_{J}\left\{h_{J}^{-1}, V(v, \epsilon)^{\frac{1}{2}}\right\}\right) \operatorname{tr}\left(\tau_{m} h_{M}\left\{h_{M}^{-1}, V\left(v^{\prime}, \epsilon\right)^{\frac{1}{2}}\right\}\right) \\
& \times \operatorname{tr}\left(\tau_{k} h_{K}\left\{h_{K}^{-1}, V(v, \epsilon)^{\frac{1}{2}}\right\}\right) \operatorname{tr}\left(\tau_{n} h_{N}\left\{h_{N}^{-1}, V\left(v^{\prime}, \epsilon\right)^{\frac{1}{2}}\right\}\right) \chi_{\epsilon}(x, y) \chi_{\epsilon}(x, v) \chi_{\epsilon}\left(x, v^{\prime}\right) .
\end{aligned}
$$

Replacing $\pi$ by $-i \hbar \kappa \delta / \delta \phi$, Poisson brackets by commutators times $1 /(i \hbar)$, and substituting $V \rightarrow \hat{V}, H_{\mathrm{kin}, \phi}(N)$ can be quantized as

$$
\begin{aligned}
\hat{H}_{\mathrm{kin}, \phi}(N)_{\gamma}= & \frac{(-i)^{2} 2^{22}}{i^{6} 3^{2} \hbar^{4} \kappa^{4} \beta^{6}} \lim _{\epsilon \rightarrow 0} \sum_{\substack{v, v^{\prime}, v^{\prime \prime}, v^{\prime \prime \prime} \in V(\gamma)\\
}} N\left(v^{\prime \prime}\right) X\left(v^{\prime \prime}\right) X\left(v^{\prime \prime \prime}\right) \chi_{\epsilon}\left(v^{\prime \prime}, v^{\prime \prime \prime}\right) \chi_{\epsilon}\left(v^{\prime \prime}, v\right) \chi_{\epsilon}\left(v^{\prime \prime}, v^{\prime}\right) \\
& \left.\times \frac{1}{E(v) E\left(v^{\prime}\right)} \sum_{\substack{s_{I} \cap s_{J} \cap s_{K}=v \\
s_{L} \cap s_{M} \cap s_{N}=v^{\prime}}} \epsilon^{I J K} \epsilon^{L M N} \epsilon_{i j k} \epsilon_{l m n}\left(\frac{1}{2}\right) \hat{e}_{I}^{i}(v)^{\left(\frac{1}{2}\right)} \hat{e}_{L}^{l}\left(v^{\prime}\right)^{\left(\frac{1}{2}\right)} \hat{e}_{J}^{j}(v)^{\left(\frac{1}{2}\right)}\right)_{M}^{m}\left(v^{\prime}\right)^{\left(\frac{1}{2}\right)} \hat{e}_{K}^{k}(v)^{\left(\frac{1}{2}\right)} \hat{e}_{N}^{n}\left(v^{\prime}\right),
\end{aligned}
$$

where $X(v):=\frac{1}{2}\left[X_{R}(v)+X_{L}(v)\right]$ is the sum of left- and right-invariant vector fields acting on the point holomomies $U(v)$ defined in [56], and

$\left(\frac{1}{2}\right) \hat{e}_{I}^{i}(v):=\operatorname{tr}\left(\tau_{i} h_{I}\left[h_{I}^{-1}, \hat{V}^{\frac{1}{2}}\right]\right)=-\operatorname{tr}\left(\tau_{i} h_{I} \hat{V}^{\frac{1}{2}} h_{I}^{-1}\right)$

is $\epsilon$-independent for sufficiently small $\epsilon$. For sufficiently small $\epsilon$, the three characteristic functions in (6.6) vanish unless $v=v^{\prime}=v^{\prime \prime}=v^{\prime \prime \prime}$. Taking the limit $\epsilon \rightarrow 0$, we obtain

$$
\hat{H}_{\mathrm{kin}, \phi}(N)_{\gamma}=\frac{2^{23}}{3 \hbar^{4} \kappa^{4} \beta^{6}} \sum_{v \in V(\gamma)} \frac{N(v)}{E(v)^{2}} X(v) X(v) \sum_{\substack{s_{I} \cap s_{J} \cap s_{K}=v \\ s_{L} \cap s_{M} \cap s_{N}=v}} \epsilon^{I J K} \epsilon^{L M N} \delta_{i l}\left(\frac{1}{2}\right) \hat{e}_{I}^{i}(v)^{\left(\frac{1}{2}\right)} \hat{e}_{L}^{l}(v) \delta_{j m}
$$




$$
\times\left(\frac{1}{2}\right) \hat{e}_{J}^{j}(v)^{\left(\frac{1}{2}\right)} \hat{e}_{M}^{m}(v) \delta_{k n}\left(\frac{1}{2}\right) \hat{e}_{K}^{k}(v)^{\left(\frac{1}{2}\right)} \hat{e}_{N}^{n}(v)=: \frac{2^{23}}{3 \hbar^{4} \kappa^{4} \beta^{6}} \sum_{v \in V(\gamma)} \frac{N(v)}{E(v)^{2}} X(v) X(v) \widehat{V}^{-1} \text { alt }, v,
$$

where we have used $\epsilon_{i j k} \epsilon_{l m n}=3 ! \delta_{[l}^{i} \delta_{m}^{j} \delta_{n]}^{k}$. The operator $\widehat{V^{-1}}$ alt, $v$ is the quantum version of $\frac{1}{\epsilon^{3} \sqrt{\operatorname{det}(q)}(x)}=\frac{1}{V(x, \epsilon)}$ up to a constant, and thus it is called the inverse volume operator. By introducing the manifestly gauge invariant operators [27]

$\hat{q}_{I J}(v):=\delta_{i j}^{\left(\frac{1}{2}\right)} \hat{e}_{I}^{i}(v)^{\left(\frac{1}{2}\right)} \hat{e}_{J}^{j}(v)$,

the inverse volume operator $\widehat{V^{-1}}$ alt, $v$ can be represented in terms of $\hat{q}_{I J}(v)$ as

$\widehat{V^{-1}}$ alt,$v \cdot f_{\gamma}=\sum_{\substack{s_{I} \cap s_{J} \cap s_{K}=v \\ s_{L} \cap s_{M} \cap s_{N}=v}} \epsilon^{I J K} \epsilon^{L M N} \hat{q}_{I L}(v) \hat{q}_{J M}(v) \hat{q}_{K N}(v) \cdot f_{\gamma}$.

Note that the operator $\hat{q}_{I J}(v)$ can be represented in terms of $\tau_{\mu}$ as

$$
\begin{aligned}
\hat{q}_{I J}(v) & =\delta^{i j} \operatorname{tr}\left(\tau_{i} h_{I} \hat{V}^{\frac{1}{2}} h_{I}^{-1}\right) \operatorname{tr}\left(\tau_{j} h_{J} \hat{V}^{\frac{1}{2}} h_{J}^{-1}\right)=-\operatorname{tr}\left(\tau_{\mu^{\prime}} h_{I} \hat{V}^{\frac{1}{2}} h_{I}^{-1}\right) C_{(1)}^{\mu^{\prime} \mu} \operatorname{tr}\left(\tau_{\mu} h_{J} \hat{V}^{\frac{1}{2}} h_{J}^{-1}\right) \\
& =-\left[\operatorname{tr}\left(\tau_{\mu} h_{I} \hat{V}^{\frac{1}{2}} h_{I}^{-1}\right)\right]^{\dagger} \operatorname{tr}\left(\tau_{\mu} h_{J} \hat{V}^{\frac{1}{2}} h_{J}^{-1}\right)=:-\left[\left(\frac{1}{2}\right) \hat{e}_{I}^{\mu}(v)\right]^{\dagger}\left(\frac{1}{2}\right) \hat{e}_{J}^{\mu}(v),
\end{aligned}
$$

where we have used the identity (4.8) and $C_{(1)}^{\mu^{\prime} \mu}=C_{(1)}^{\mu \mu^{\prime}} \equiv(-1)^{1+\mu} \delta_{\mu,-\mu^{\prime}}$ in the second step and the following identities in the third step:

$\overline{\left(h_{I}\right)_{B}^{A}}=\left(h_{I}^{-1}\right)_{A}^{B}, \quad \overline{\left(\tau_{i}\right)^{A}{ }_{B}}=-\left(\tau_{i}\right)_{A}^{B}, \quad \overline{\left(\tau_{\mu}\right)_{B}^{A}}=\left(\tau_{\mu^{\prime}}\right)_{A}^{B} C_{(1)}^{\mu^{\prime} \mu}$.

Here the overline denotes complex conjugation.

6.2 The action of $\widehat{V^{-1}}$ alt, $v$ on a trivalent non-planar vertex

We consider the action of $\widehat{V^{-1}}$ alt, $v$ on $T_{\gamma, \vec{j}, \vec{i}}^{v, s}(A)$ at a trivalent non-planar vertex $v$. Notice that the intertwiner space associated to $v$, which will be acted by $\widehat{V}^{-1}$ alt, $v$, is of one dimension. Hence the gauge-invariant operators $\hat{q}_{I J}(v)$ and $\widehat{V}^{-1}$ alt, $v$ take eigenvalues on the orthonormal spin-network states

$T_{\gamma, \vec{j}, \vec{i}}^{v, s, \text { norm }}(A):=\sqrt{d_{j_{1}} d_{j_{2}} d_{j_{3}}} T_{\gamma, \vec{j}, \vec{i}}^{v, s}(A)$

Therefore we have

$$
\begin{aligned}
& \hat{q}_{I J}(v) \cdot T_{\gamma, \vec{j}, \vec{i}}^{v, s, \operatorname{norm}}(A)=Q_{I J} T_{\gamma, \vec{j}, \vec{i}}^{v, s, \operatorname{norm}}(A), \\
& \widehat{V^{-1}} \text { alt }, v \cdot T_{\gamma, \vec{j}, \vec{i}}^{v, s, \text { norm }}(A)=\epsilon^{I J K} \epsilon^{L M N} Q_{I L} Q_{J M} Q_{K N} T_{\gamma, \vec{j}, \vec{i}}^{v, s, \text { norm }}(A),
\end{aligned}
$$

where

$Q_{I J}=\left(T_{\gamma, \vec{j}, \vec{i}}^{v, s, \text { norm }}, \hat{q}_{I J}(v) \cdot T_{\gamma, \vec{j}, \vec{i}}^{v, s, \text { norm }}\right)_{\mathcal{H}_{\text {kin }}}=-\left(\left(\frac{1}{2}\right) \hat{e}_{I}^{\mu}(v) \cdot T_{\gamma, \vec{j}, \vec{i}}^{v, s, \text { norm }},\left(\frac{1}{2}\right) \hat{e}_{J}^{\mu}(v) \cdot T_{\gamma, \vec{j}, \vec{i}}^{v, s, \text { norm }}\right)_{\mathcal{H}_{\text {kin }}}$.

In order to obtain the eigenvalues $Q_{I J}$, we need to calculate the action of $\left(\frac{1}{2}\right) \hat{e}_{I}^{\mu}(v)$ on $T_{\gamma, \vec{j}, \vec{i}}^{v, s, \text { norm }}(A)$ or $T_{\gamma, \vec{j}, \vec{i}}^{v, s}(A)$. In the following, we will only display the derivation of the two components $Q_{11}$ and $Q_{12}$, and the remaining components of $Q_{I J}$ can be written down similarly. 
Now let us consider the action of $\left(\frac{1}{2}\right) \hat{e}_{1}^{\mu}(v)$ on $T_{\gamma, \vec{j}, \vec{i}}^{v, s}(A)$. Notice that the spherical tensors $\tau_{\mu}$ can be represented as Eq. (3.21). Hence we have

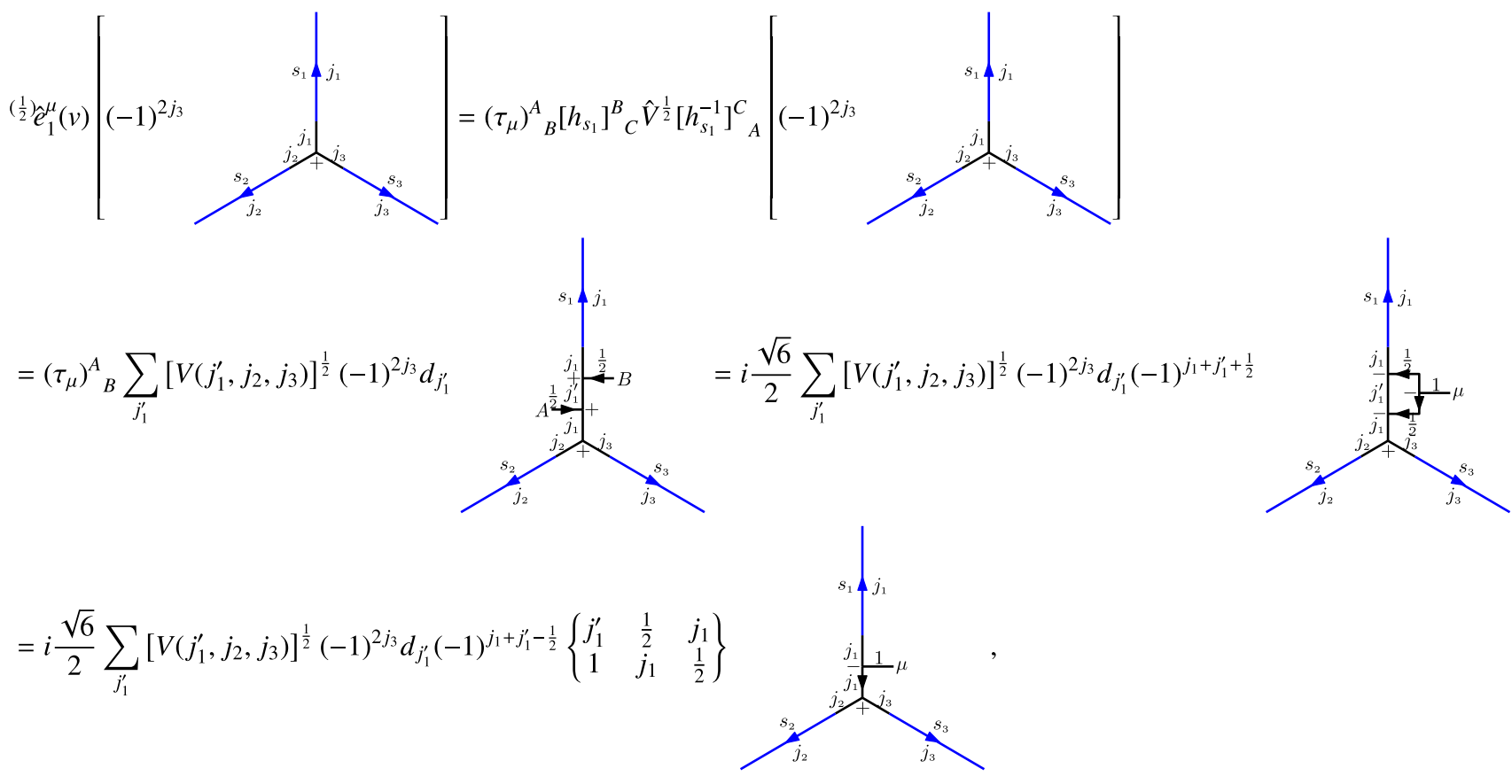

where in the second step we have used the result of Eq. (5.18), and in the fourth step used the identity (see B.5 for a proof)

$\begin{array}{lll}j_{1} & \frac{1}{2} \\ j_{1}^{\prime} & -1 \\ j_{1} & \frac{1}{2}\end{array}=-\left\{\begin{array}{ccc}j_{1}^{\prime} & \frac{1}{2} & j_{1} \\ 1 & j_{1} & \frac{1}{2}\end{array}\right\} \frac{j_{1}}{j_{1}} \uparrow 1$

Taking account of

$d_{j_{1}^{\prime}}(-1)^{j_{1}+j_{1}^{\prime}-\frac{1}{2}}\left\{\begin{array}{ccc}j_{1}^{\prime} & \frac{1}{2} & j_{1} \\ 1 & j_{1} & \frac{1}{2}\end{array}\right\}=\frac{2}{\sqrt{6}} \sqrt{\frac{j_{1}\left(j_{1}+1\right)}{2 j_{1}+1}}\left\{\begin{array}{ll}1, & j_{1}^{\prime}=j_{1}-\frac{1}{2} \\ -1, & j_{1}^{\prime}=j_{1}+\frac{1}{2}\end{array}\right.$,

Eq. (6.17) can be reduced to

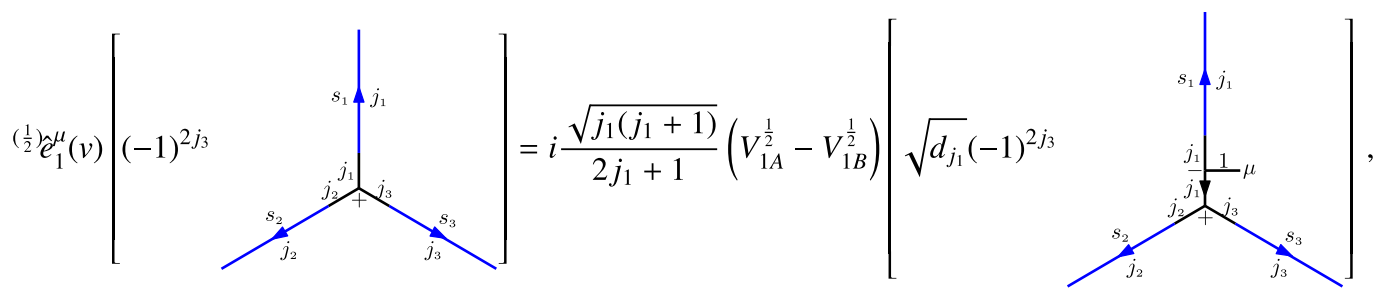

where

$V_{1 A}^{\frac{1}{2}}:=\left[V\left(j_{1}^{\prime}=j_{1}-1 / 2, j_{2}, j_{3}\right)\right]^{\frac{1}{2}}, \quad V_{1 B}^{\frac{1}{2}}:=\left[V\left(j_{1}^{\prime}=j_{1}+1 / 2, j_{2}, j_{3}\right)\right]^{\frac{1}{2}}$. 
The intertwiner in Eq. (6.19) is normalized because of

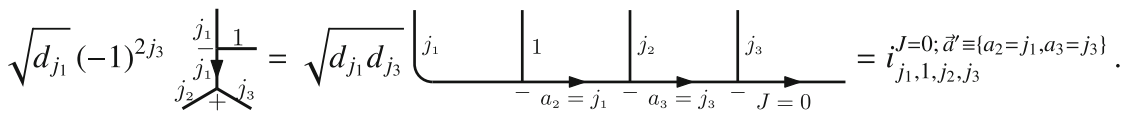

Equation (6.19) implies that $\hat{e}_{1}^{\mu}(v)$ changes neither the graph nor the spins of $T_{\gamma, \vec{j}, \vec{i}}^{v, s}(A)(5.13)$. But it does change the intertwiner $i_{v} \equiv i_{j_{1}, j_{2}, j_{3}}^{J=\left\{\vec{a} \equiv\left\{a_{2}=j_{3}\right\}\right.}$ in Eq. (5.9) into $i_{v}^{\prime} \equiv i_{j_{1}, 1, j_{2}, j_{3}}^{J=0, \vec{a}^{\prime} \equiv\left\{a_{2}=j_{1}, a_{3}=j_{3}\right\}}$ in Eq. (6.21) associated to $v$. Hence we obtain

$$
\begin{aligned}
& Q_{11}=-\left({ }^{\left(\frac{1}{2}\right)} \hat{e}_{1}^{\mu}(v) T_{\gamma, \vec{j}, \vec{i}}^{v, s, \text { norm }},\left(\frac{1}{2}\right) \hat{e}_{1}^{\mu}(v) T_{\gamma, \vec{j}, \vec{i}}^{v, s, \text { norm }}\right)_{\mathcal{H}_{\text {kin }}} \\
& =-\left(i \frac{\sqrt{j_{1}\left(j_{1}+1\right)}}{2 j_{1}+1}\left(V_{1 A}^{\frac{1}{2}}-V_{1 B}^{\frac{1}{2}}\right) T_{\gamma, \vec{j}, \bar{i}^{\prime}}^{v, s, \text { norm }}, i \frac{\sqrt{j_{1}\left(j_{1}+1\right)}}{2 j_{1}+1}\left(V_{1 A}^{\frac{1}{2}}-V_{1 B}^{\frac{1}{2}}\right) T_{\gamma, \vec{j}, i^{\prime}}^{v, s, \text { norm }}\right)_{\mathcal{H}_{k i n}} \\
& =-\frac{j_{1}\left(j_{1}+1\right)}{\left(2 j_{1}+1\right)^{2}}\left(V_{1 A}^{\frac{1}{2}}-V_{1 B}^{\frac{1}{2}}\right)^{2} \int_{S U(2)^{3}} \prod_{I=1,2,3} \mathrm{~d} \mu_{H}\left(h_{S_{I}}\right) \overline{T_{\gamma, \vec{j}, \vec{i}^{\prime}}^{v, s, n o r m}(A)} T_{\gamma, \vec{j}, i^{\prime}}^{v, s, \text { norm }}(A) \\
& =-\frac{j_{1}\left(j_{1}+1\right)}{\left(2 j_{1}+1\right)^{2}}\left(V_{1 A}^{\frac{1}{2}}-V_{1 B}^{\frac{1}{2}}\right)^{2} \operatorname{tr}\left(\overline{i_{j_{1}, 1, j_{2}, j_{3}}^{J=0 ; \vec{a}^{\prime} \equiv\left\{a_{2}=j_{1}, a_{3}=j_{3}\right\}}} \cdot i_{j_{1}, 1, j_{2}, j_{3}}^{J=0 ; \vec{a}^{\prime} \equiv\left\{a_{2}=j_{1}, a_{3}=j_{3}\right\}}\right) \\
& =-\frac{j_{1}\left(j_{1}+1\right)}{\left(2 j_{1}+1\right)^{2}}\left(V_{1 A}^{\frac{1}{2}}-V_{1 B}^{\frac{1}{2}}\right)^{2} \operatorname{tr}\left(i_{j_{1}, 1, j_{2}, j_{3}}^{J=0 ; \vec{a}^{\prime} \equiv\left\{a_{2}=j_{1}, a_{3}=j_{3}\right\}} \cdot i_{j_{1}, 1, j_{2}, j_{3}}^{J=0 ; \vec{a}^{\prime}=\left\{a_{2}=j_{1}, a_{3}=j_{3}\right\}}\right) \\
& =-\frac{j_{1}\left(j_{1}+1\right)}{\left(2 j_{1}+1\right)^{2}}\left(V_{1 A}^{\frac{1}{2}}-V_{1 B}^{\frac{1}{2}}\right)^{2} \text {, }
\end{aligned}
$$

where $\operatorname{tr}()$ denotes contracting magnetic quantum numbers, we have integrated holonomies to give the contraction of the intertwiner with its complex conjugate in the fourth step and used the fact that the intertwiner is real in the fifth step, and the intertwiner is normalized in the last step.

Similarly, the action of $\hat{e}_{2}^{\mu}(v)$ on $T_{\gamma, \vec{j}, \vec{i}}^{v, s}(A)$ yields

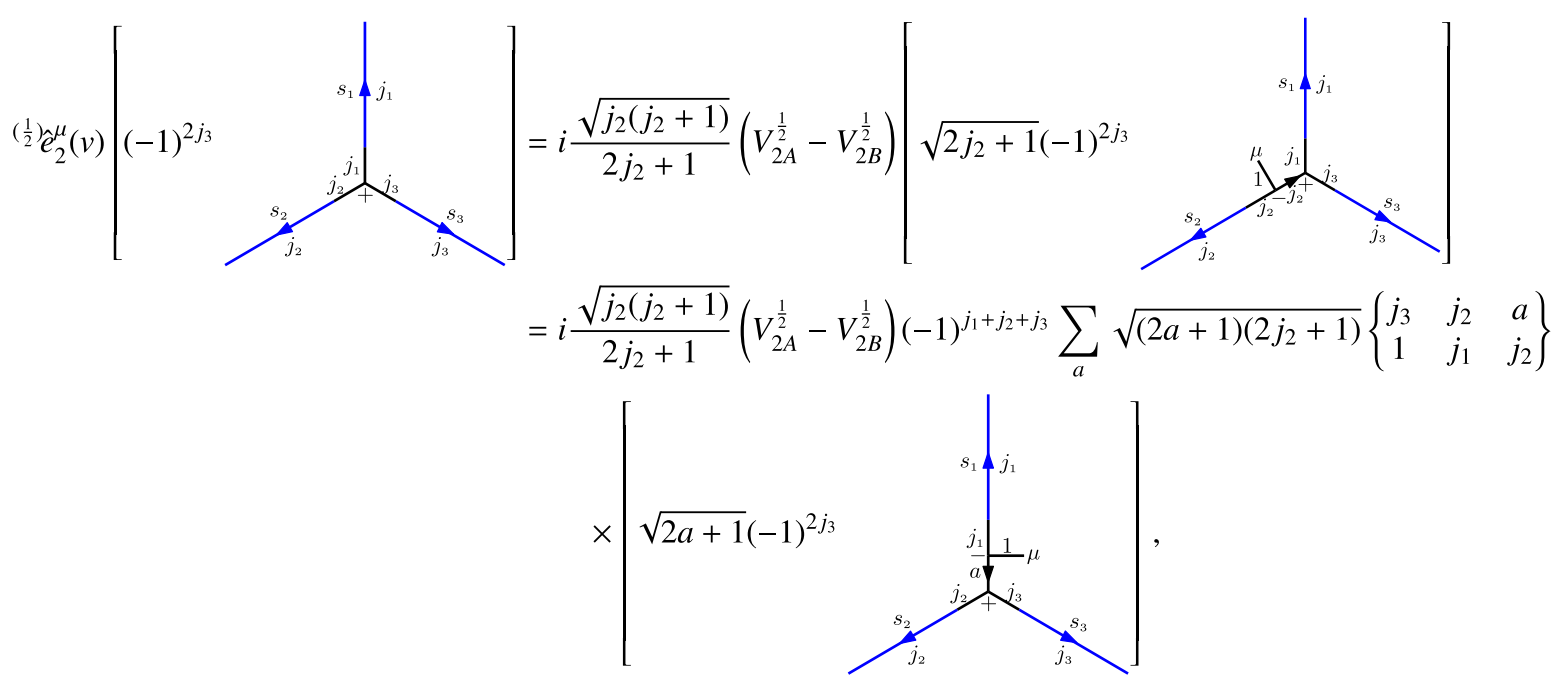

where

$V_{2 A}^{\frac{1}{2}}:=\left[V\left(j_{2}^{\prime}=j_{2}-1 / 2, j_{3}, j_{1}\right)\right]^{\frac{1}{2}}, \quad V_{2 B}^{\frac{1}{2}}:=\left[V\left(j_{2}^{\prime}=j_{2}+1 / 2, j_{3}, j_{1}\right)\right]^{\frac{1}{2}}$, 
and in the second step we have used the following identity (see Appendix B.5 for a proof):

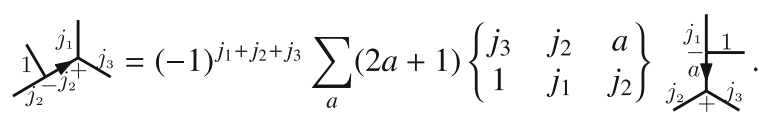

The intertwiner in Eq. (6.23) is also normalized because of

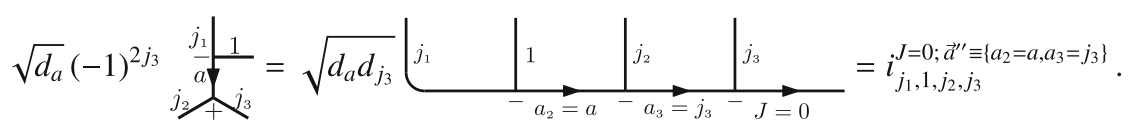

Hence $^{\left(\frac{1}{2}\right)} \hat{e}_{2}^{\mu}(v)$ changes $i_{v} \equiv i_{j_{1}, j_{2}, j_{3}}^{J=\left\{\vec{a} \equiv\left\{a_{2}=j_{3}\right\}\right.}$ in Eq. (5.9) into composition of $i_{v}^{\prime \prime} \equiv i_{j_{1}, 1, j_{2}, j_{3}}^{J=0 ; a^{\prime \prime} \equiv\left\{a_{2}=a, a_{3}=j_{3}\right\}}$ in Eq. (6.26) associated to $v$. Finally, we have

$$
\begin{aligned}
& Q_{12}=-\left(\left(\frac{1}{2}\right) \hat{e}_{1}^{\mu}(v) \cdot T_{\gamma, \vec{j}, \vec{i}}^{v, s, \text { norm }},\left(\frac{1}{2}\right) \hat{e}_{2}^{\mu}(v) \cdot T_{\gamma, \vec{j}, \vec{i}}^{v, s, \text { norm }}\right)_{\mathcal{H}_{k i n}}=-\frac{\sqrt{j_{1}\left(j_{1}+1\right)}}{2 j_{1}+1}\left(V_{1 A}^{\frac{1}{2}}-V_{1 B}^{\frac{1}{2}}\right) \\
& \times \frac{\sqrt{j_{2}\left(j_{2}+1\right)}}{2 j_{2}+1}\left(V_{2 A}^{\frac{1}{2}}-V_{2 B}^{\frac{1}{2}}\right)(-1)^{j_{1}+j_{2}+j_{3}} \sum_{a} \sqrt{(2 a+1)\left(2 j_{2}+1\right)} \\
& \times\left\{\begin{array}{ccc}
j_{3} & j_{2} & a \\
1 & j_{1} & j_{2}
\end{array}\right\} \operatorname{tr}\left(i_{j_{1}, 1, j_{2}, j_{3}}^{J=0 ; \vec{a}^{\prime} \equiv\left\{a_{2}=j_{1}, a_{3}=j_{3}\right\}} \cdot i_{j_{1}, 1, j_{2}, j_{3}}^{J=0 ; a_{2}^{\prime \prime} \equiv\left\{a_{2}=a, a_{3}=j_{3}\right\}}\right) \\
& =-\frac{\sqrt{j_{1}\left(j_{1}+1\right) j_{2}\left(j_{2}+1\right)}}{\left(2 j_{1}+1\right)\left(2 j_{2}+1\right)}\left(V_{1 A}^{\frac{1}{2}}-V_{1 B}^{\frac{1}{2}}\right)\left(V_{2 A}^{\frac{1}{2}}-V_{2 B}^{\frac{1}{2}}\right)(-1)^{j_{1}+j_{2}+j_{3}} \sum_{a} \sqrt{(2 a+1)\left(2 j_{2}+1\right)}\left\{\begin{array}{ccc}
j_{3} & j_{2} & a \\
1 & j_{1} & j_{2}
\end{array}\right\} \delta_{a, j_{1}} \\
& =-\frac{\sqrt{j_{1}\left(j_{1}+1\right) j_{2}\left(j_{2}+1\right)}}{\left(2 j_{1}+1\right)\left(2 j_{2}+1\right)}\left(V_{1 A}^{\frac{1}{2}}-V_{1 B}^{\frac{1}{2}}\right)\left(V_{2 A}^{\frac{1}{2}}-V_{2 B}^{\frac{1}{2}}\right)(-1)^{j_{1}+j_{2}+j_{3}} \sqrt{\left(2 j_{1}+1\right)\left(2 j_{2}+1\right)}\left\{\begin{array}{ccc}
j_{3} & j_{2} & j_{1} \\
1 & j_{1} & j_{2}
\end{array}\right\} \\
& =-\frac{\sqrt{j_{1}\left(j_{1}+1\right) j_{2}\left(j_{2}+1\right)}}{\left(2 j_{1}+1\right)\left(2 j_{2}+1\right)}\left(V_{1 A}^{\frac{1}{2}}-V_{1 B}^{\frac{1}{2}}\right)\left(V_{2 A}^{\frac{1}{2}}-V_{2 B}^{\frac{1}{2}}\right) s(-1)^{j_{1}+j_{2}+j_{3}} \sqrt{\left(2 j_{1}+1\right)\left(2 j_{2}+1\right)}(-1)^{j_{1}+j_{2}+j_{3}+1} \\
& \times \frac{2\left[j_{1}\left(j_{1}+1\right)+j_{2}\left(j_{2}+1\right)-j_{3}\left(j_{3}+1\right)\right]}{\sqrt{2 j_{1}\left(2 j_{1}+1\right)\left(2 j_{1}+2\right) 2 j_{2}\left(2 j_{2}+1\right)\left(2 j_{2}+2\right)}} \\
& =\frac{j_{1}\left(j_{1}+1\right)+j_{2}\left(j_{2}+1\right)-j_{3}\left(j_{3}+1\right)}{2\left(2 j_{1}+1\right)\left(2 j_{2}+1\right)}\left(V_{1 A}^{\frac{1}{2}}-V_{1 B}^{\frac{1}{2}}\right)\left(V_{2 A}^{\frac{1}{2}}-V_{2 B}^{\frac{1}{2}}\right) \text {. }
\end{aligned}
$$

Similarly we can write down the remaining components of $Q_{I J}$ and thus the eigenvalue of $\widehat{V}^{-1}$ alt, $v$ in (6.15).

\section{Summary and discussion}

In the previous sections, the graphical method developed by Yutsis and Brink and their extensions, which suit the requirement of representing the holonomies and the intertwiners, are applied to LQG. The algebraic formula is represented by its corresponding graphical formula in an unique and unambiguous way. Then the matrix elements of the operator $\hat{q}_{I J K}$, which is the basic building block of the volume operator, are calculated via the simple rules of transforming graphs. Note that the calculations that we did by the graphical method can also be performed by conventional algebraic techniques. Also, corresponding to every graphical reduction, there is an algebraic reduction because of the correspondence between the graphical and algebraic formulas. However, it is obvious that the graphical method is more concise, intuitive and visual.

Note that in our graphical representation, a gauge-invariant intertwiner associated to a vertex $v$ of a standard graph at which $n$ edges with spin $j_{1}, \ldots, j_{n}$ incident is represented by

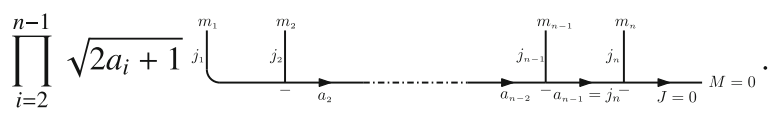


Taking account of Eqs. (A.47) and (A.42), Eq. (7.1) is equal to $(-1)^{2 j_{n}}$ times of the formula

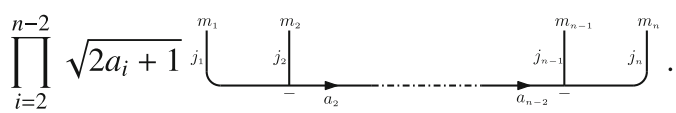

Since the only difference between (7.2) and (7.1) is a factor $(-1)^{2 j_{n}}$, Eq. (7.2) is also used to represent the gauge-invariant intertwiner in the literature.

Let us compare our calculation with those existing in the literature. The operator $\hat{q}_{I J K}$ can be represented by the following three forms [11]:

$\hat{q}_{I J K}:=-4 i \epsilon_{i j k} J_{e_{I}}^{i} J_{e_{J}}^{j} J_{e_{K}}^{k}=4\left[J_{e_{I}}^{i} J_{e_{J}}^{i}, J_{e_{J}}^{j} J_{e_{K}}^{j}\right]=\left[\left(J_{e_{I}}^{i}+J_{e_{J}}^{i}\right)^{2},\left(J_{e_{J}}^{j}+J_{e_{K}}^{j}\right)^{2}\right]$.

The first and the third forms (equalities) of the expression (7.3) were adopted as the starting points, respectively, in [18] and in [11,19], and their matrix elements are calculated by graphical and algebraic methods, respectively. In this paper, we considered the second expression (equality) of $\hat{q}_{I J K}$ and derived its matrix elements by the graphical method introduced in Sect. 3. In [18], to compute the closed formula, Pietri and Rovelli adopted the Kauffman's graphical method to deal with recoupling problems. Note that the idea in [18] to employ the first equality of (7.3) to calculate the volume operator can also be carried out by the unique and unambiguous rule of graphical calculation. From (3.5), we have

$$
\begin{aligned}
\hat{q}_{I J K} \cdot\left(i_{v}^{J ; \vec{a}}\right)_{m_{1} \cdots m_{I} \cdots m_{J} \cdots m_{K} \cdots m_{n}} & =-4 i \epsilon_{i j k} J_{e_{I}}^{i} J_{e_{J}}^{j} J_{e_{K}}^{k} \cdot\left(i_{v}^{J ; \vec{a}}\right)_{m_{1} \cdots m_{I} \cdots m_{J} \cdots m_{K} \cdots m_{n}}^{M} \\
& =\left(i_{v}^{J ; \vec{a}}\right)_{m_{1} \cdots m_{I}^{\prime} \cdots m_{J}^{\prime} \cdots m_{K}^{\prime} \cdots m_{n}}{ }^{M} 4 \epsilon_{i j k}\left[\pi_{j_{I}}\left(\tau_{i}\right)\right]^{m_{I}^{\prime}{ }_{m_{I}}}\left[\pi_{j_{J}}\left(\tau_{j}\right)\right]^{m_{J}^{\prime}}{ }_{m_{J}}\left[\pi_{j_{K}}\left(\tau_{k}\right)\right]^{m_{K}^{\prime}}{ }_{m_{K}} \\
& =\left(i_{v}^{J ; \vec{a}}\right)_{m_{1} \cdots m_{I}^{\prime} \cdots m_{J}^{\prime} \cdots m_{K}^{\prime} \cdots m_{n}} \stackrel{M}{ }(-4 i) \epsilon_{\mu v \rho}\left[\pi_{j_{I}}\left(\tau_{\mu}\right)\right]^{m_{I}^{\prime}}{ }_{m_{I}}\left[\pi_{j_{J}}\left(\tau_{v}\right)\right]^{m_{J}^{\prime}}{ }_{m_{J}}\left[\pi_{j_{K}}\left(\tau_{\rho}\right)\right]^{m_{K}^{\prime}}{ }_{m_{K}},
\end{aligned}
$$

where, in the last step, we have used the following identity (see Appendix B.6 for a proof):

$\epsilon_{i j k}\left[\pi_{j_{I}}\left(\tau_{i}\right)\right]^{m_{I}^{\prime}{ }_{m_{I}}}\left[\pi_{j_{J}}\left(\tau_{j}\right)\right]_{m_{J}}^{m_{J}^{\prime}}\left[\pi_{j_{K}}\left(\tau_{k}\right)\right]^{m_{K}^{\prime}}{ }_{m_{K}}=-i \epsilon_{\mu \nu \rho}\left[\pi_{j_{I}}\left(\tau_{\mu}\right)\right]_{m_{I}}^{m_{I}^{\prime}}\left[\pi_{j_{J}}\left(\tau_{\nu}\right)\right]^{m_{J}^{\prime}}{ }_{m_{J}}\left[\pi_{j_{K}}\left(\tau_{\rho}\right)\right]^{m_{K}^{\prime}}{ }_{m_{K}}$,

with

$\epsilon_{\mu \nu \rho}=\sqrt{6}\left(\begin{array}{lll}1 & 1 & 1 \\ \mu & \nu & \rho\end{array}\right)$

Note that here one has $\epsilon_{-10+1}=1$ (see also Appendix B.6 for a proof). Notice that both $\left[\pi_{j}\left(\tau_{\mu}\right)\right]^{m^{\prime}}{ }_{m}$ and $\epsilon_{\mu \nu \rho}$ (given by a special $3 j$-symbol) in Eq. (7.4) have corresponding graphical representations. The action of $\hat{q}_{I J K}$, corresponding to (7.4), on an intertwiner is given by

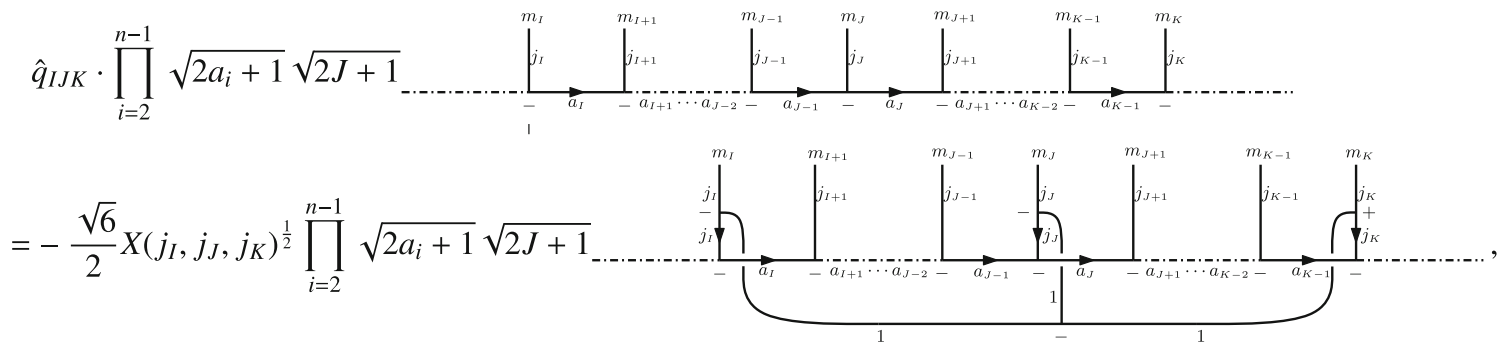

where $X\left(j_{I}, j_{J}, j_{K}\right) \equiv 2 j_{I}\left(2 j_{I}+1\right)\left(2 j_{I}+2\right) 2 j_{J}\left(2 j_{J}+1\right)\left(2 j_{J}+2\right) 2 j_{K}\left(2 j_{K}+1\right)\left(2 j_{K}+2\right)$. The derivation of the action of $\hat{q}_{I J K}$ on the intertwiner in the graphical method is to remove the three curves with spin 1 in (7.7) by using the previous 
rules of transforming graphs. The identities in Eqs. (4.14), (4.15), (4.16) and (4.17) enable us to reduce the graphical formula (7.7) as

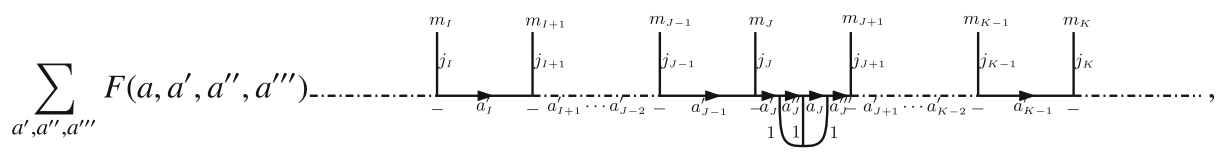

where the factor $F\left(a, a^{\prime}, a^{\prime \prime}, a^{\prime \prime \prime}\right)$ involves the intermediate momenta $a, a^{\prime}, a^{\prime \prime}$ and $a^{\prime \prime \prime}$ in the intertwiner. By the graphical identity

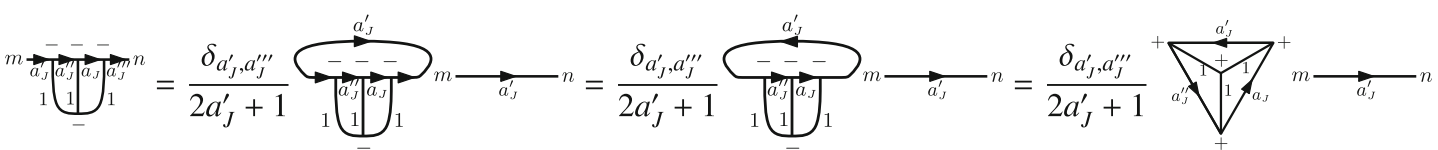

$$
\begin{aligned}
& =\frac{\delta_{a_{J}^{\prime}, a_{J}^{\prime \prime \prime}}}{2 a_{J}^{\prime}+1}\left\{\begin{array}{ccc}
1 & 1 & 1 \\
a_{J} & a_{J}^{\prime} & a_{J}^{\prime \prime}
\end{array}\right\} m \longrightarrow_{a_{J}^{\prime}}{ }^{n},
\end{aligned}
$$

we can remove the three curves with spin 1 and obtain the final result, which coincides with (4.23). The closed formula of the volume operator was also derived by Brunnemann and Thiemann in $[11,19]$ using the algebraic techniques. The derivation process in [11,19] is rigorous but rather abstract and awkward. Our graphical method is convenient and visual, and our result (4.27) coincides with the formula derived by the algebraic calculation for the case of $I>1$ and $J>I+1$ in [19]. Moreover, our analysis shows that Eq. (4.27) is also valid for other cases and hence can be regarded as a general expression.

In principle, in the light of the matrix elements of $\hat{q}_{I J K}$ in Eq. (4.27) we can finally write down the action of the volume operator on the spin-network states. We denote

$\hat{q}_{v} \equiv \frac{i \ell_{\mathrm{p}}^{6} \beta^{3}}{8 \times 4} \sum_{I<J<K, e_{I} \cap e_{J} \cap e_{K}=v} \varsigma\left(e_{I}, e_{J}, e_{K}\right) \hat{q}_{I J K}$.

With the matrix elements of $\hat{q}_{I J K}$, we can get the eigenvalues and corresponding eigenstates of $\hat{q}_{v}$ as

$\hat{q}_{v}\left|\lambda_{\hat{q}_{v}}\right\rangle=\lambda_{\hat{q}_{v}}\left|\lambda_{\hat{q}_{v}}\right\rangle$

Then we can write down the action of $\hat{V}_{v}$ on the intertwiner $\left|i_{v}\right\rangle$ associated to $v$ as

$\hat{V}_{v}\left|i_{v}\right\rangle=\sqrt{\left|\hat{q}_{v}\right|}\left|i_{v}\right\rangle=\sum_{\lambda_{\hat{q}_{v}}} \sqrt{\left|\hat{q}_{v}\right|}\left|\lambda_{\hat{q}_{v}}\right\rangle\left\langle\lambda_{\hat{q}_{v}} \mid i_{v}\right\rangle=\sum_{\lambda_{\hat{q}_{v}}}\left[\sqrt{\left|\lambda_{\hat{q}_{v}}\right|}\left\langle\lambda_{\hat{q}_{v}} \mid i_{v}\right\rangle\right]\left|\lambda_{\hat{q}_{v}}\right\rangle$

However, when the dimension of the intertwiner space associated to $v$ is bigger than nine, one cannot diagonalize $\hat{q}_{v}$ analytically. This prevents us from explicitly writing down the whole formula for the action of $\hat{V}_{v}$.

Since the volume operator is defined only by the flux operator (essentially the self-adjoint right-invariant operator). Hence the derivation of the closed formula for the matrix element of volume operator just involves the action of the self-adjoint right-invariant operator on spin-network states. The action involves the recoupling problem and can be dealt smoothly by simple rules of transforming graphs in the Brink original graphical method. Comparing to volume operator, the gravitational Hamiltonian constraint operator and the inverse volume operator depend also on holonomies in addition to fluxes. In order to calculate their actions on spin-network states in graphical method, we have to extend Brink's representation and propose a graphical representation and calculation for the holonomy. The corresponding graphical representation and calculation including holonomy is thus proposed in this paper, which enables us to do the graphical calculus uniformly in Brink's original graphical framework. The action of the Euclidean Hamiltonian $\hat{H}_{v}^{E}$ on the spin network states $T_{\gamma, \vec{j}, \vec{i}}^{v, s}(A)$ with trivalent vertex $v$ was shown in (5.28). The difference between our result (5.26) and (II.14) in [14] is the factor $1 / 2$. Since the factor is overall, the qualitative conclusion made in [14] is not affected by the missing factor $1 / 2$.

In the general case of matter fields coupled to gravity, the 3-metric $q_{a b}$ enters the Hamiltonian of matters. For instance, the information of gravity is encoded in the inverse of volume function in (6.2). In classical cosmological models, the 
Hamiltonian of matters will diverse at the big bang singularity (with zero volume). However, in LQC the inverse volume operator corresponding to the inverse of the scale factor is bounded above [25]. To see whether the boundedness of the inverse scale factor operator in LQC is maintained by the inverse volume operator in LQG, the expectation values of the inverse volume operator $\widehat{V^{-1}}$ alt,$v$ with respect to gauge-invariant states at a trivalent vertex, which is a non-trivial eigenstate with zero-eigenvalue, was calculated in LQG $[27,57]$. The conclusion drawn in [27,57] is that $\widehat{V}^{-1}$ alt, $v$ is unbounded. To crosscheck the algebraic calculation in [27], the same action of $\widehat{V}^{-1}$ alt, $v$ has been calculated by graphical method in this paper. Based on the gauge invariant operators $\hat{q}_{I J}(v)$, the inverse volume operator $\widehat{V^{-1}}$ alt,$v$ defined in (6.10) takes eigenvalues on the orthonormal spin network state $T_{\gamma, \vec{j}, \vec{i}}^{v, s, \text { norm }}(A)$. The eigenvalues of $\widehat{V^{-1}}$ alt, $v$, presented in (6.15), consist of the eigenvalues $Q_{I J}$ of $\hat{q}_{I J}(v)$. The different conclusions between Ref. [27] and ours come from the different eigenvalues $Q_{I J}$ on the same state $T_{\gamma, \vec{j}, i}^{v, s, \text { norm }}$. More concretely, there are two differences on $Q_{I J}$ : (i) a global sign and (ii) the coefficients of $Q_{I J}$. It turns out that there are two mistakes made in [27], which lead to the incorrect value of $Q_{I J}$. First, a minus sign was missed in the second step of Eq. (4.5) in [27], namely, the right formula should be $\left[\hat{e}_{I}^{i}(v)\right]^{\dagger}=-\hat{e}_{I}^{i}(v)$ rather than $\left[\hat{e}_{I}^{i}(v)\right]^{\dagger}=\hat{e}_{I}^{i}(v)$, which is the reason of (i). Second, the values of coefficients $C_{\tilde{J}}^{J j_{K}} \tilde{j}_{K}\left(A, M, g_{N-1}\right)$ defined in Eq. (3.4) in [27] were incorrect, namely, the factor $(-1)^{2 j_{K}}$ there should be replaced by 1. Hence the values of $Q_{I J}$ in Eq. (4.17) of [27] should be corrected. By taking the above two corrections and taking account of the different definitions of $\tau_{i}$ (differing for each other by the factor 2 ), the algebraic calculation would give the same results of $Q_{I J}$ as in this paper. There are similar corrections for other values of $Q_{I J}$. These corrections lead to a significant change of the conclusion, namely, the eigenvalue $\epsilon^{I J K} \epsilon^{L M N} Q_{I L} Q_{J M} Q_{K N}$ of $\widehat{V^{-1}}$ alt,$v$ on $T_{\gamma, \vec{j}, \vec{i}}^{v, s}(A)$ is indeed zero. In other words, on the contrary to the conclusion in [27], our calculation shows that the inverse volume operator $\widehat{V^{-1}}$ alt,$v$ is bounded (zero-valued) at a trivalent non-planar vertex of the gauge-invariant spin-network states. This conclusion coincides with the one made in [58], although different quantum versions of volume function are adopted.

In principle, the graphical calculation method can be applied to the general cases, where the spin-network states are defined on arbitrarily valent vertices and the holonomies appearing in the two operators are expressed in an arbitrary representation of the gauge group. However, for those general cases, the volume operator lacks the explicit matrix elements formula. This prevents us from doing further calculations. For the same reason, the matrix elements of the Lorentzian part of the full gravitational Hamiltonian constraint operator have not been explicitly written down even on the trivalent vertices except for certain special cases [23].

Acknowledgements The authors would like to thank Antonia Zipfel for helpful discussions. J. Y. would also like to thank Chopin Soo and Hoi-Lai Yu for useful discussions. J. Y. is supported in part by NSFC Grant No. 11347006, by the Institute of Physics, Academia Sinica, Taiwan, and by the Natural Science Foundation of Guizhou University (Grant No. 47 in 2013). Y. M. is supported in part by the NSFC (Grants No. 11475023 and No. 11235003) and the Research Fund for the Doctoral Program of Higher Education of China.

Open Access This article is distributed under the terms of the Creative Commons Attribution 4.0 International License (http://creativecomm ons.org/licenses/by/4.0/), which permits unrestricted use, distribution, and reproduction in any medium, provided you give appropriate credit to the original author(s) and the source, provide a link to the Creative Commons license, and indicate if changes were made.

Funded by SCOAP ${ }^{3}$.

\section{Appendix A: Elements of graphical representation and calculation}

\section{A.1 Representations of SU(2), Clebsch-Gordan decomposition, and the intertwiner}

To every non-negative integer or half-integer $j$ (i.e., for $j=0, \frac{1}{2}, 1, \frac{3}{2}, \ldots$ ), there exists an irreducible representation $\pi_{j}$ of $S U$ (2), specified by $j$, on a Hilbert space $\mathcal{H}_{j}$ with dimension $2 j+1$. The orthonormal basis of $\mathcal{H}_{j}$ may be denoted by $\left\{e_{m}^{(j)}\right\}$, or $\{|j m\rangle\}$ in Dirac's notation, where $m=-j,-j+1, \ldots, j$. Given two irreducible representations $\pi_{j_{1}}$ and $\pi_{j_{2}}$ of $S U(2)$ on $\mathcal{H}_{j_{1}}$ and $\mathcal{H}_{j_{2}}$, the tensor product representation $\pi_{j_{1}} \otimes \pi_{j_{2}}$ on $\mathcal{H}_{j_{1}} \otimes \mathcal{H}_{j_{2}}$ is $\left(2 j_{1}+1\right)\left(2 j_{2}+1\right)$-dimensional reducible representation of $S U(2)$. The Clebsch-Gordan theorem tells us that the representation $\pi_{j_{1}} \otimes \pi_{j_{2}}$ can be decomposed into a direct sum of irreducible representations $\pi_{J}$ on $\mathcal{H}_{J}$, where $J \in\left\{\left|j_{1}-j_{2}\right|, \ldots, j_{1}+j_{2}\right\}$, formally,

$I_{j_{1} j_{2}}\left(\pi_{j_{1}}(g) \otimes \pi_{j_{2}}(g)\right)\left(I_{j_{1} j_{2}}\right)^{-1}=\bigoplus_{J=\left|j_{1}-j_{2}\right|}^{j_{1}+j_{2}} \pi_{J}(g), \quad \forall g \in S U(2)$, 
where $I_{j_{1} j_{2}}$ is called the intertwining operator in the representation theory of groups [39]. Given bases $e_{m_{1}}^{\left(j_{1}\right)} \otimes e_{m_{2}}^{\left(j_{2}\right)} \in \mathcal{H}_{j_{1}} \otimes \mathcal{H}_{j_{2}}$ (or $\left|j_{1} m_{1} j_{2} m_{2}\right\rangle \equiv\left|j_{1} m_{1}\right\rangle \otimes\left|j_{2} m_{2}\right\rangle \in \mathcal{H}_{j_{1}} \otimes \mathcal{H}_{j_{2}}$ ) and $e_{M}^{(J)} \in \mathcal{H}_{J}$ (or $|J M\rangle \in \mathcal{H}_{J}$ ), the components of $I_{j_{1} j_{2}}$, as matrix elements, are given by

$$
\begin{aligned}
& \left(I_{j_{1} j_{2}}\right)_{m_{1} m_{2}}^{J M}=\left(e_{M}^{(J)}, e_{m_{1}}^{\left(j_{1}\right)} \otimes e_{m_{2}}^{\left(j_{2}\right)}\right) \mathcal{H}_{j_{1}} \otimes \mathcal{H}_{j_{2}}=e_{(J)}^{M}\left(e_{m_{1}}^{\left(j_{1}\right)} \otimes e_{m_{2}}^{\left(j_{2}\right)}\right), \\
& \quad \text { or }\left(I_{j_{1} j_{2}}\right)_{m_{1} m_{2}}{ }^{J M}=\left\langle J M \mid j_{1} m_{1} j_{2} m_{2}\right\rangle
\end{aligned}
$$

where $e_{(J)}^{M}$ is the dual basis or the basis of $\mathcal{H}_{J}^{*} \cdot\left(I_{j_{1} j_{2}}\right)_{m_{1} m_{2}}{ }^{J M}$ are called the complex conjugates of the Clebsch-Gordan coefficients (CGCs) or the intertwiners. In matrix form, the row and column indices of $I_{j_{1} j_{2}}$ are denoted by the latter (upper) and former (down) indices $J M$ and $m_{1} m_{2}$, respectively. Our convention enables us to regard $\left(I_{j_{1} j_{2}}\right)_{m_{1} m_{2}} J M$ as components of the intertwiner tensor whose indices can be lowered and raised by a "metric" which will be introduced by (A.6). For given $j_{1}, j_{2}$ and $J$, we denote $\left(I_{j_{1} j_{2}}^{J}\right)_{m_{1} m_{2}}^{M} \equiv\left(I_{j_{1} j_{2}}\right)_{m_{1} m_{2}}{ }^{J M}$ which projects the bases $e_{m_{1}}^{\left(j_{1}\right)} \otimes e_{m_{2}}^{\left(j_{2}\right)}$ of $\mathcal{H}_{j_{1}} \otimes \mathcal{H}_{j_{2}}$ onto $e_{M}^{(J)}$ of $\mathcal{H}_{J} \subset \mathcal{H}_{j_{1}} \otimes \mathcal{H}_{j_{2}}$. The corresponding matrix elements of representations $\pi_{j_{1}} \otimes \pi_{j_{2}}$ and $\pi_{J}$ in the two bases, respectively, are related to each other by the so-called Clebsch-Gordan series

$\sum_{m_{1}, m_{2}, n_{1}, n_{2}}\left(I_{j_{1} j_{2}}^{J}\right)_{m_{1} m_{2}}{ }^{M}\left[\pi_{j_{1}}(g)\right]_{n_{1}}^{m_{1}}\left[\pi_{j_{2}}(g)\right]_{n_{2}}^{m_{2}}\left(\left(I_{j_{1} j_{2}}^{J}\right)^{-1}\right)_{N}^{n_{1} n_{2}}=\left[\pi_{J}(g)\right]_{N}^{M}$.

The representation $\pi_{j}$ of $S U(2)$ on $\mathcal{H}_{j}$ induces a conjugate representation $\pi_{j}^{*}$ of $S U(2)$ on $\mathcal{H}_{j}^{*}$ via

$\left[\pi_{j}(g)^{*} e_{(j)}^{m}\right]\left(e_{n}^{(j)}\right):=e_{(j)}^{m}\left(\pi_{j}\left(g^{-1}\right) e_{n}^{(j)}\right)$

where $\left\{e_{(j)}^{m}\right\}$ is the orthogonal basis of $\mathcal{H}_{j}^{*}$. Furthermore, the irreducible and unitary properties of $\pi_{j}$ are preserved to its conjugate representation $\pi_{j}^{*}$. If the representation $\pi_{j}$ is unitary, there exists an unitary operator $C^{(j)}: \mathcal{H}_{j} \rightarrow \mathcal{H}_{j}^{*}$, such that [42]

$C^{(j)} \pi_{j}(g)=\pi_{j}(g)^{*} C^{(j)} \Leftrightarrow C^{(j)} \pi(g) C^{(j)^{-1}}=\pi_{j}(g)^{*}$.

The operator $C^{(j)}$ in fact defines an isomorphism between $\mathcal{H}_{j}$ and $\mathcal{H}_{j}^{*}$ by $e_{m}^{(j)}=C_{m m^{\prime}}^{(j)} e_{(j)}^{m^{\prime}}$ and $e_{(j)}^{m}=C_{(j)}^{m m^{\prime}} e_{m^{\prime}}^{(j)}$, where $C_{(j)}^{m n} \equiv\left(C^{(j)^{-1}}\right)^{m n}$. The operator $C^{(j)}$ and its inverse $C^{(j)^{-1}}$ play an important role also in quantum field theories, whose components in the bases of $\mathcal{H}_{j}$ and $\mathcal{H}_{j}^{*}$ are given by [42]

$C_{m n}^{(j)}:=(-1)^{j-n} \delta_{n,-m}=(-1)^{j+m} \delta_{m,-n}$,

$C_{(j)}^{m n} \equiv\left(C^{(j)^{-1}}\right)^{m n}:=(-1)^{j-m} \delta_{m,-n}=(-1)^{j+n} \delta_{n,-m}$,

satisfying

$C_{m n}^{(j)} C_{(j)}^{n m^{\prime}}=C_{(j)}^{m^{\prime} n} C_{n m}^{(j)}=\delta_{m}^{m^{\prime}}$,

$C_{n m}^{(j)} C_{(j)}^{n m^{\prime}}=C_{(j)}^{m^{\prime} n} C_{m n}^{(j)}=(-1)^{2 j} \delta_{m}^{m^{\prime}}$.

Obviously, $C_{m n}^{(j)}$ and $C_{(j)}^{m n}$ satisfy

$C_{m^{\prime} m}^{(j)}=(-1)^{2 j} C_{m m^{\prime}}^{(j)}, \quad C_{(j)}^{m m^{\prime}}=(-1)^{2 j} C_{(j)}^{m^{\prime} m}$, 
which implies that $C_{m n}^{(j)}$ and $C_{(j)}^{m n}$ are symmetric for integer $j$, and anti-symmetric for half-odd integer $j$. The operator $C^{(j)}$ and its inverse $C^{(j)^{-1}}$ can be used to lower and raise indices of the tensors on $\mathcal{H}_{j}$. Hence $C_{m n}^{(j)}$ behaves like a metric tensor. Equation (A.5) can be written in the form of its components as

$C_{m m^{\prime}}^{(j)}\left[\pi_{j}(g)\right]_{n^{\prime}}^{m^{\prime}} C_{(j)}^{n^{\prime} n}=\left[\pi_{j}(g)^{*}\right]_{m}{ }^{n}$

The fact that the representation $\pi_{j}$ is unitary implies

$\left[\pi_{j}\left(g^{-1}\right)\right]_{m}^{n}=\left[\pi_{j}(g)^{-1}\right]_{m}^{n}=\left[\pi_{j}(g)^{\dagger}\right]_{m}^{n}=\overline{\left[\pi_{j}(g)\right]_{n}^{m}}=\left[\pi_{j}(g)^{*}\right]_{m}{ }^{n}=C_{m m^{\prime}}^{(j)}\left[\pi_{j}(g)\right]^{m^{\prime}}{ }_{n^{\prime}} C_{(j)}^{n^{\prime} n}$,

where the overline denotes complex conjugation, and we have used (A.4) and (A.11) in the last two steps. By the map $C^{(j)}$ we can define a natural inner product on $\mathcal{H}_{j}^{*}$ as $\left(C^{(j)} f, C^{(j)} g\right)_{\mathcal{H}_{j}^{*}}:=(g, f)_{\mathcal{H}_{j}}, \forall f, g \in \mathcal{H}_{j}$. Then the base transformation $I_{j_{1}, j_{2}}^{J}$ in $\mathcal{H}_{j_{1}} \otimes \mathcal{H}_{j_{2}}$ induces the base transformation $I_{j_{1}, j_{2}}^{*}$ in $\mathcal{H}_{j_{1}}^{*} \otimes \mathcal{H}_{j_{2}}^{*}$ by

$\left(I_{j_{1}, j_{2}}^{*}\right)_{J M}^{m_{1} m_{2}}=\overline{\left(I_{j_{1} j_{2}}\right)_{m_{1} m_{2}}{ }^{J M}}=\sum_{m_{1}^{\prime}, m_{2}^{\prime}} C_{\left(j_{1}\right)}^{m_{1} m_{1}^{\prime}} C_{\left(j_{2}\right)}^{m_{2} m_{2}^{\prime}}\left(I_{j_{1} j_{2}}\right)_{m_{1}^{\prime} m_{2}^{\prime}}{ }^{J M^{\prime}} C_{M^{\prime} M}^{(J)}$,

Now let us consider the decomposition of the tensor product $\pi_{j_{1}} \otimes \cdots \otimes \pi_{j_{n}}$ of irreducible representations of $S U$ (2) for $n>2$. The composition involves $n-1$ decompositions of the tensor products of two representations as (A.1) and the choice of the decomposition schemes. Denote $a_{i}(i=2, \ldots, n-1)$ the irreducible representations that appeared in the $i$ th decomposition for a given scheme. In the following, we consider the standard scheme where we firstly decompose $\pi_{j_{1}} \otimes \pi_{j_{2}}$ into $\bigoplus \pi_{a_{2}}$, and then decompose $\pi_{a_{2}} \otimes \pi_{j_{3}}$ into $\bigoplus \pi_{a_{3}}$, and so on. We also denote $\vec{a} \equiv\left\{a_{2}, \ldots a_{n-1}\right\}$. For given $j_{1} \ldots, j_{n}$, allowable $J$, and compatible vector $\vec{a} \equiv\left\{a_{2}, \ldots a_{n-1}\right\}$, the corresponding Clebsch-Gordan series reads

$\sum_{m_{1}, \ldots, m_{n}, n_{1}, \ldots, n_{n}}\left(I_{j_{1} \cdots j_{n}}^{J ; \vec{a}}\right)_{m_{1} \cdots m_{n}}{ }^{M}\left[\pi_{j_{1}}(g)\right]_{n_{1}}^{m_{1}} \cdots\left[\pi_{j_{n}}(g)\right]_{n_{n}}^{m_{n}}\left(\left(I_{j_{1} \cdots j_{n}}^{J ; \vec{a}}\right)^{-1}\right)_{N}^{n_{1} \cdots n_{n}}=\left[\pi_{J}(g)\right]_{N}^{M}$,

where $\left(I_{j_{1} \cdots j_{n}}^{J ; \vec{a}}\right)_{m_{1} \cdots m_{n}}^{M} \equiv\left(I_{j_{1} \cdots j_{n}}^{\vec{a}}\right)_{m_{1} \cdots m_{n}}{ }^{J M} \equiv \sum_{k_{2}, \ldots, k_{n-1}}\left(I_{j_{1} j_{2}}\right)_{m_{1} m_{2}} a_{2} k_{2} \ldots\left(I_{a_{n-1} j_{n}}\right)_{k_{n-1} m_{n}} J M$ are the general CGCs, the intertwiners, and often rewritten in quantum mechanics in the form $\left\langle J M ; \vec{a} \mid j_{1} m_{1} j_{2} m_{2} \cdots j_{n} m_{n}\right\rangle=\sum_{k_{2}, \ldots, k_{n-1}}$ $\left\langle a_{2} k_{2} \mid j_{1} m_{1} j_{2} m_{2}\right\rangle \cdots\left\langle J M \mid a_{n-1} k_{n-1} j_{n} m_{n}\right\rangle$. For the convenience of a graphical representation, we introduce

$\left(\begin{array}{c}J ; \vec{a} \\ j_{j_{1} \cdots j_{n}}\end{array}\right)_{m_{1} \cdots m_{n}}^{M}:=(-1)^{j_{1}-\sum_{i=2}^{n} j_{i}-J}\left(\begin{array}{c}J ; \vec{a} \\ I_{j_{1} \cdots j_{n}}\end{array}\right)_{m_{1} \cdots m_{n}}^{M}=(-1)^{j_{1}-\sum_{i=2}^{n} j_{i}-J}\left\langle J M ; \vec{a} \mid j_{1} m_{1} j_{2} m_{2} \cdots j_{n} m_{n}\right\rangle$.

Notice that the factor $(-1)^{j_{1}-\sum_{i=2}^{n} j_{i}-J}$ in Eq. (A.15) involves only the spins $j_{1}, \ldots, j_{n}$ and $J$, not the intermediate momenta $a_{2}, \ldots, a_{n-1}$. From now on, the intertwiners refer in particular to $i_{j_{1} \cdots j_{n}}^{J ; \vec{a}}$. The Clebsch-Gordan series (A.14) can be written in terms of $i_{j_{1} \cdots j_{n}}^{J ; \vec{a}}$ as

$\sum_{m_{1}, \ldots, m_{n}, n_{1}, \ldots, n_{n}}\left(i_{j_{1} \cdots j_{n}}^{J ; \vec{a}}\right)_{m_{1} \cdots m_{n}}{ }^{M}\left[\pi_{j_{1}}(g)\right]_{n_{1}}^{m_{1}} \cdots\left[\pi_{j_{n}}(g)\right]_{n_{n}}^{m_{n}}\left(\left(i_{j_{1} \cdots j_{n}}^{J ; \vec{a}}\right)^{-1}\right)_{N}^{n_{1} \cdots n_{n}}=\left[\pi_{J}(g)\right]_{N}^{M}$

and its inverse reads

$\left[\pi_{j_{1}}(g)\right]_{n_{1}}^{m_{1}} \cdots\left[\pi_{j_{n}}(g)\right]_{n_{n}}^{m_{n}}=\sum_{J, M, N}\left(\left(i_{j_{1} \cdots j_{n}}^{J ; \vec{a}}\right)^{-1}\right)_{M}^{m_{1} \cdots m_{n}}\left[\pi_{J}(g)\right]_{N}^{M}\left(i_{j_{1} \cdots j_{n}}^{J ; \vec{a}}\right)_{n_{1} \cdots n_{n}}$.

It is easy to generalize the above results to the decomposition of the tensor product $\pi_{j_{1}} \otimes \cdots \otimes \pi_{j_{k}}(g) \otimes \pi_{j_{k+1}}(g)^{-1} \otimes \cdots \otimes$ $\pi_{j_{n}}(g)^{-1}$ of $k$ representations and $n-k$ inverse representations into a direct sum of irreducible representations $\pi_{J}$ on $\mathcal{H}_{J}$. The corresponding Clebsch-Gordan series read 


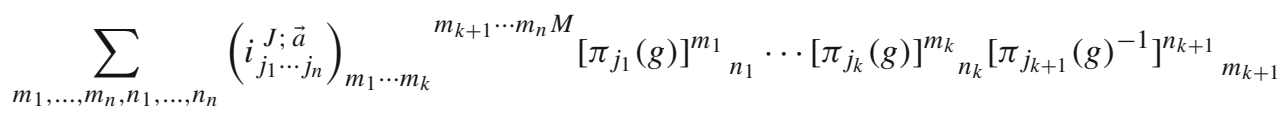

$$
\begin{aligned}
& \cdots\left[\pi_{j_{n}}(g)^{-1}\right]^{n_{n}}{ }_{m_{n}}\left(\left(i_{j_{1} \cdots j_{n}}^{J ; \vec{a}}\right)^{-1}\right)_{N n_{k+1} \cdots n_{n}}{ }^{n_{1} \cdots n_{k}}=\left[\pi_{J}(g)\right]^{M}{ }_{N},
\end{aligned}
$$

where

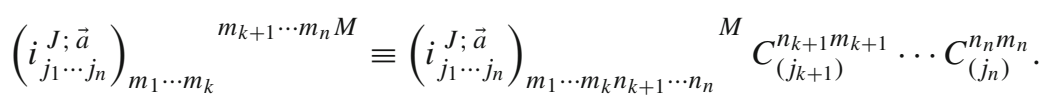

Equation (A.18) can be written as

$$
\begin{aligned}
& \sum_{m_{1}, \ldots, m_{n}}\left[\pi_{j_{k+1}}(g)^{-1}\right]^{n_{k+1}}{ }_{m_{k+1}} \cdots\left[\pi_{j_{n}}(g)^{-1}\right]_{m_{n}}^{n_{n}}\left(i_{j_{1} \cdots j_{n}} ; \dot{\vec{a}}\right)_{m_{1} \cdots m_{k}}{ }^{m_{k+1} \cdots m_{n} M}\left[\pi_{j_{1}}(g)\right]^{m_{1}}{ }_{n_{1}} \\
& \cdots\left[\pi_{j_{k}}(g)\right]^{m_{k}}{ }_{n_{k}}=\sum_{N}\left[\pi_{J}(g)\right]^{M}{ }_{N}\left(i_{j_{1} \cdots j_{n}}^{J ; \vec{a}}\right)_{n_{1} \cdots n_{k}}{ }_{n_{k+1} \cdots n_{n} N}
\end{aligned}
$$

which, in the case of $J=0$, reduces to

$$
\begin{aligned}
& \sum_{m_{1}, \ldots, m_{n}}\left[\pi_{j_{k+1}}(g)^{-1}\right]^{n_{k+1}}{ }_{m_{k+1}} \cdots\left[\pi_{j_{n}}(g)^{-1}\right]_{m_{n}}^{n_{n}}\left(I_{j_{1} \cdots j_{n}}^{0 ; \vec{a}}\right)_{m_{1} \cdots m_{k}}{ }^{m_{k+1} \cdots m_{n} 0}{ }_{\left[\pi_{j_{1}}(g)\right]^{m_{1}}}{ }_{n_{1}} \cdots\left[\pi_{j_{k}}(g)\right]^{m_{k}}{ }_{n_{k}} \\
& =\left(I_{j_{1} \cdots j_{n}}^{0 ; \vec{a}}\right)_{n_{1} \cdots n_{k}}{ }^{n_{k+1} \cdots n_{n} 0} .
\end{aligned}
$$

Hence the tensor $\left(\begin{array}{c}0 ; \vec{a} \\ i_{j_{1} \cdots j_{n}}\end{array}\right)_{m_{1} \cdots m_{k}} m_{k+1} \cdots m_{n} 0$ is also called the invariant tensor. In the special case of $n=2$, the Clebsch-Gordan series reads

$\left[\pi_{j_{1}}(g)\right]_{n_{1}}^{m_{1}}\left[\pi_{j_{2}}(g)\right]_{n_{2}}^{m_{2}}=\sum_{J, M, N}\left(\left(i_{j_{1} j_{2}}^{J}\right)^{-1}\right)_{M}^{m_{1} m_{2}}\left[\pi_{J}(g)\right]_{N}^{M}\left(i_{j_{1} j_{2}}^{J}\right)_{n_{1} n_{2}}{ }^{N}$.

The fact that the operator $i_{j_{1} \cdots j_{n}}^{J ; \vec{a}}$ is unitary and its matrix elements take real numbers results in $\left(\left(i_{j_{1} \cdots j_{n}}^{J ; \vec{a}}\right)^{-1}\right)_{M}^{m_{1} \cdots m_{n}}=$ $\left(\left(i_{j_{1} \cdots j_{n}}^{J ; \vec{a}}\right)^{\dagger}\right)_{M}^{m_{1} \cdots m_{n}}=\left(i_{j_{1} \cdots j_{n}}^{J ; \vec{a}}\right)_{m_{1} \cdots m_{n}}{ }^{M}$.

Given $n$ angular momenta $j_{1}, \ldots, j_{n}$, the intertwiner space $\mathcal{H}_{j_{1}, \ldots, j_{n}}$ consists of the intertwiners $\left(i_{j_{1} \cdots j_{n}}^{J ; \vec{a}}\right)_{m_{1} \cdots m_{n}} \quad{ }^{M}$ with the following inner product:

$$
\begin{aligned}
& \left\langle\vec{a}^{\prime} ; J^{\prime}, M^{\prime} \mid \vec{a} ; J, M\right\rangle_{\mathcal{H}_{j_{1}, \ldots, j_{n}}} \equiv \sum_{m_{1}, \ldots, m_{n}}\left(\left(i_{j_{1} \cdots j_{n}}^{J^{\prime} ; \vec{a}^{\prime}}\right)_{m_{1} \cdots m_{n}}{ }^{M^{\prime}},\left(i_{j_{1} \cdots j_{n}}^{J ; \vec{a}}\right)_{m_{1} \cdots m_{n}}{ }^{M}\right)_{\mathcal{H}_{j_{1}, \ldots, j_{n}}} \\
& :=\sum_{m_{1}, \ldots, m_{n}}\left(\left(i_{j_{1} \cdots j_{n}}^{J^{\prime} ; \vec{a}^{\prime}}\right)^{\dagger}\right)_{M^{\prime}}^{m_{1} \cdots m_{n}}\left(\begin{array}{c}
\left.i_{j_{1} \cdots j_{n}}^{J ; \vec{a}}\right)_{m_{1} \cdots m_{n}} \\
M
\end{array}\right. \\
& =\sum_{m_{1}, \ldots, m_{n}} \overline{\left(i_{j_{1} \cdots j_{n}}^{J^{\prime} ; \vec{a}^{\prime}}\right)_{m_{1} \cdots m_{n}}{ }^{M \prime}}\left(i_{j_{1} \cdots j_{n}}^{J ; \vec{a}}\right)_{m_{1} \cdots m_{n}} M \\
& =\delta_{J, J^{\prime}} \delta_{M, M^{\prime}} \delta_{\vec{a}, \vec{a}^{\prime}},
\end{aligned}
$$

where the last step can be arrived by using the fact that the matrix $\left(\underset{j_{1} \cdots j_{n}}{J ; \vec{a}}\right)_{m_{1} \cdots m_{n}}{ }^{M}$ is unitary. 
A.2 The basic components of the graphical representation and simple rules of transforming graphs

In this subsection, we introduce the basic components of the graphical representation and simple rules of transforming graphs [31]. A graphical representation for the matrix elements of irreducible representations of $S U$ (2) is also proposed. A graphical representation is a correspondence between graphical and algebraic formulas. Each term in an algebraic formula is represented by a component of an appropriate graph in a unique and unambiguous way.

The Wigner $3 j$-symbol is associated with the coupling of three angular momenta to give a zero resultant. The $3 j$-symbol has simple symmetric properties and hence is easier to handle than the CGC. The $3 j$-symbol is defined in terms of the CGC by $[41,42]$

$$
\left(\begin{array}{ccc}
j_{1} & j_{2} & j_{3} \\
m_{1} & m_{2} & m_{3}
\end{array}\right)=\frac{(-1)^{j_{1}-j_{2}-m_{3}}}{\sqrt{2 j_{3}+1}}\left\langle j_{3}-m_{3} \mid j_{1} m_{1} j_{2} m_{2}\right\rangle=\frac{(-1)^{j_{1}-j_{2}-j_{3}}}{\sqrt{2 j_{3}+1}} \sum_{m_{3}^{\prime}}\left\langle j_{3} m_{3}^{\prime} \mid j_{1} m_{1} j_{2} m_{2}\right\rangle C_{m_{3} m_{3}^{\prime}}^{\left(j_{3}\right)}
$$

or

$$
\left\langle j_{3} m_{3} \mid j_{1} m_{1} j_{2} m_{2}\right\rangle=(-1)^{j_{1}-j_{2}-j_{3}} \sqrt{2 j_{3}+1} \sum_{m_{3}^{\prime}}\left(\begin{array}{ccc}
j_{1} & j_{2} & j_{3} \\
m_{1} & m_{2} & m_{3}^{\prime}
\end{array}\right) C_{\left(j_{3}\right)}^{m_{3}^{\prime} m_{3}} .
$$

The $3 j$-symbol takes non-vanishing value when the parameters of the upper row $\left(j_{1}, j_{2}, j_{3}\right)$ satisfy the triangular condition (i.e., $\left.\left|j_{1}-j_{2}\right| \leqslant j_{3} \leqslant j_{1}+j_{2}\right)$ and when the sum of the parameters of the lower row $\left(m_{1}, m_{2}, m_{3}\right)$ is zero. The parameters $j_{i}$ and $m_{i}$ are simultaneously integers or half-integers, such that each of the numbers

$j_{i}+m_{i}, \quad j_{i}-m_{i}, \quad j_{1}+j_{2}+j_{3},-j_{1}+j_{2}+j_{3}, \quad j_{1}-j_{2}+j_{3}, \quad j_{1}+j_{2}-j_{3}$,

takes some integer. The $3 j$-symbol has the following properties. An even permutation of the columns leaves the numerical value unchanged, while an odd permutation is equivalent to a multiplication by $(-1)^{j_{1}+j_{2}+j_{3}}$, i.e.,

$$
\left(\begin{array}{ccc}
j_{1} & j_{2} & j_{3} \\
m_{1} & m_{2} & m_{3}
\end{array}\right)=(-1)^{j_{1}+j_{2}+j_{3}}\left(\begin{array}{ccc}
j_{1} & j_{3} & j_{2} \\
m_{1} & m_{3} & m_{2}
\end{array}\right)
$$

Moreover, the $3 j$-symbol has the symmetric property

$$
\left(\begin{array}{ccc}
j_{1} & j_{2} & j_{3} \\
m_{1} & m_{2} & m_{3}
\end{array}\right)=(-1)^{j_{1}+j_{2}+j_{3}}\left(\begin{array}{ccc}
j_{1} & j_{2} & j_{3} \\
-m_{1} & -m_{2} & -m_{3}
\end{array}\right)
$$

which reflects the fact that the $3 j$-symbol takes real numbers (dues to the real CGCs), i.e.,

$$
\begin{aligned}
& \left(\begin{array}{ccc}
j_{1} & j_{2} & j_{3} \\
m_{1} & m_{2} & m_{3}
\end{array}\right)=\sum_{m_{1}^{\prime}, m_{2}^{\prime}, m_{3}^{\prime}} C_{m_{1} m_{1}^{\prime}}^{\left(j_{1}\right)} C_{m_{2} m_{2}^{\prime}}^{\left(j_{2}\right)} C_{m_{3} m_{3}^{\prime}}^{\left(j_{3}\right)}\left(\begin{array}{ccc}
j_{1} & j_{2} & j_{3} \\
m_{1}^{\prime} & m_{2}^{\prime} & m_{3}^{\prime}
\end{array}\right)^{*}=\sum_{m_{1}^{\prime}, m_{2}^{\prime}, m_{3}^{\prime}} C_{m_{1} m_{1}^{\prime}}^{\left(j_{1}\right)} C_{m_{2} m_{2}^{\prime}}^{\left(j_{2}\right)} C_{m_{3} m_{3}^{\prime}}^{\left(j_{3}\right)}\left(\begin{array}{ccc}
j_{1} & j_{2} & j_{3} \\
m_{1}^{\prime} & m_{2}^{\prime} & m_{3}^{\prime}
\end{array}\right) \\
& \quad \times\left(\begin{array}{ccc}
j_{1} & j_{2} & j_{3} \\
m_{1} & m_{2} & m_{3}
\end{array}\right)=\left(\begin{array}{ccc}
j_{1} & j_{2} & j_{3} \\
m_{1} & m_{2} & m_{3}
\end{array}\right)^{*}=\sum_{m_{1}^{\prime}, m_{2}^{\prime}, m_{3}^{\prime}} C_{\left(j_{1}\right)}^{m_{1} m_{1}^{\prime}} C_{\left(j_{2}\right)}^{m_{2} m_{2}^{\prime}} C_{\left(j_{3}\right)}^{m_{3} m_{3}^{\prime}}\left(\begin{array}{ccc}
j_{1} & j_{2} & j_{3} \\
m_{1}^{\prime} & m_{2}^{\prime} & m_{3}^{\prime}
\end{array}\right)
\end{aligned}
$$

The orthogonality relation for the $3 j$-symbol is expressed as

$$
\sum_{m_{1}, m_{2}}\left(\begin{array}{ccc}
j_{1} & j_{2} & j_{3} \\
m_{1} & m_{2} & m_{3}
\end{array}\right)\left(\begin{array}{ccc}
j_{1} & j_{2} & j_{3}^{\prime} \\
m_{1} & m_{2} & m_{3}^{\prime}
\end{array}\right)=\frac{\delta_{j_{3}, j_{3}^{\prime}}}{2 j_{3}+1} \delta_{m_{3}, m_{3}^{\prime}}
$$

Furthermore, the $3 j$-symbol is normalized as

$\sum_{m_{1}, m_{2}, m_{3}}\left(\begin{array}{ccc}j_{1} & j_{2} & j_{3} \\ m_{1} & m_{2} & m_{3}\end{array}\right)\left(\begin{array}{ccc}j_{1} & j_{2} & j_{3} \\ m_{1} & m_{2} & m_{3}\end{array}\right)=1$. 
The graphical representation of the $3 j$-symbol was collected by Yutsis in [30] and slightly modified by Brink in [31] for convenience. The $3 j$-symbol is represented by an oriented node with three lines, which stand for three coupling angular momenta $j_{1}, j_{2}, j_{3}$ incident at the node [31]. The orientation of the node is meant for the cyclic order of the lines. A clockwise orientation is denoted by a - sign and an anti-clockwise orientation by a + sign. Rotation of the diagram does not change the cyclic order of lines, and the angles between two lines as well as their lengths at a node have no significance. Consequently, any geometrical deformation of the diagram which preserves the orientation of the node does not change the $3 j$-symbol represented by the diagram. The $3 j$-symbol can be written in the graphical form

$\left(\begin{array}{ccc}j_{1} & j_{2} & j_{3} \\ m_{1} & m_{2} & m_{3}\end{array}\right)=m_{1} \frac{j_{1}}{j_{2} \searrow_{m_{2}}^{j_{3}}}=m_{1} \overbrace{j_{3}}^{m_{3}} \overbrace{m_{3}}^{j_{2}}$

The property (A.27) of the $3 j$-symbol implies<smiles>CC(C)CCCCCCC(C)C</smiles>

The "metric" tensor $C_{m^{\prime} m}^{(j)}$ in Eq. (A.6), which occurs in the contraction of two $3 j$-symbols with the same $j$ values, is denoted by a line with an arrow on it as

$C_{m^{\prime} m}^{(j)}=(-1)^{j-m} \delta_{m,-m^{\prime}}=(-1)^{j+m^{\prime}} \delta_{m,-m^{\prime}}={ }_{m \longrightarrow} \stackrel{j}{\longrightarrow} m^{\prime}$,

and its inverse in Eq. (A.7) can be expressed as

$C_{(j)}^{m m^{\prime}}=(-1)^{j-m} \delta_{m,-m^{\prime}}=(-1)^{j+m^{\prime}} \delta_{m,-m^{\prime}}={ }_{m \longrightarrow} \stackrel{j}{\longrightarrow} m^{\prime}$.

A line with no arrow represents the expression

$\delta_{m, m^{\prime}}={ }_{m} \quad j$.

In a graphical representation, two lines representing the same angular momentum can be joined. Summation over a magnetic quantum number $m$ is graphically represented by joining the free ends of the corresponding lines. Equation (A.25) implies that the CGC can be represented graphically by

$\left\langle j_{3} m_{3} \mid j_{1} m_{1} j_{2} m_{2}\right\rangle=(-1)^{j_{1}-j_{2}-j_{3}} \sqrt{2 j_{3}+1} \overbrace{m_{2}}^{m_{1}} \overbrace{j_{2}}^{j_{1}} \longrightarrow m_{3}=\left.(-1)^{j_{1}-j_{2}-j_{3}} \sqrt{2 j_{3}+1} \underbrace{j_{j_{1}}^{m_{1}}}_{-j_{3}} m_{m_{3}}\right|_{j_{2}} ^{j_{2}}$

Therefore, the graphical representation of the intertwiner defined in Eq. (A.15) is

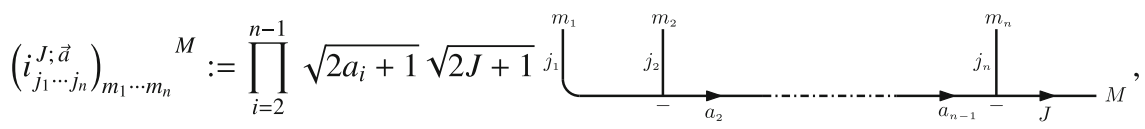

and the generalized intertwiner in Eq. (A.19) can be repressed by

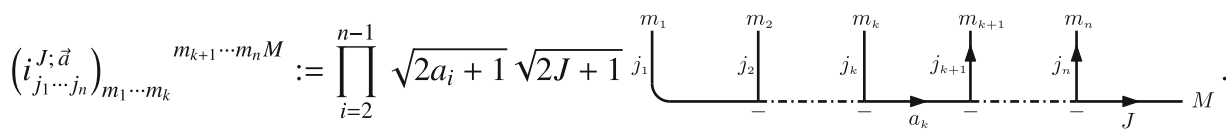


As an ingredient of the flux operator, the spherical tensor $\left[\pi_{j}\left(\tau_{\mu}\right)\right]^{m^{\prime}}{ }_{m}$ in Eq. (3.7) can be represented graphically by

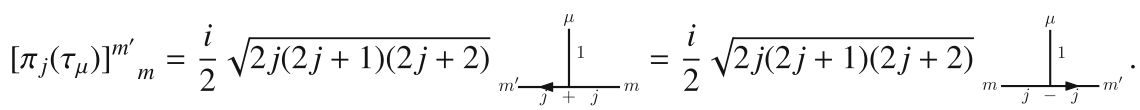

Now we outline some useful rules of transforming graphs, which can simplify our calculation of the action of operators on the quantum states in LQG. The frequently used rules for adding or removing arrows in a graph read

$\stackrel{j}{\longrightarrow} m^{\prime}={ }_{m \longrightarrow j}{ }^{j} m^{\prime}={ }_{m} \stackrel{j}{m^{\prime}}$,

$\underset{m \longrightarrow}{\longrightarrow} m^{\prime}=\stackrel{j}{\longleftarrow} m_{m}^{\prime}=(-1)^{2 j}{ }_{m} \stackrel{j}{\longleftarrow} m^{\prime}$,

$m_{m}^{j} m^{\prime}=(-1)^{2 j}{ }_{m} \stackrel{j}{m^{\prime}}$,

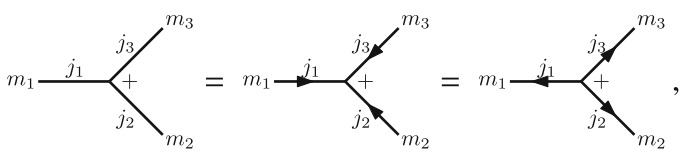

which correspond to the algebraic formulas in Eqs. (A.8), (A.9), (A.10) and (A.29). The rule to remove a closed loop in a graph reads

$m_{3} \stackrel{j_{3}}{\overbrace{j_{1}}^{j_{2}}} \overbrace{j_{3}^{\prime}}^{\prime} m_{3}^{\prime}=\frac{\delta_{j_{3}, j_{3}^{\prime}}}{2 j_{3}+1} m_{3} \longrightarrow j_{3} m_{3}^{\prime}$,

which is related to the orthogonality relation for the $3 j$-symbol in Eq. (A.30). Equation (A.31) implies

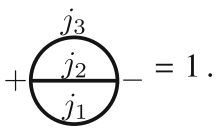

The special $3 j$-symbol with one zero-valued angular momentum is related to the "metric" tensor by

$\left(\begin{array}{ccc}j & j^{\prime} & 0 \\ m & m^{\prime} & 0\end{array}\right)=\frac{\delta_{j, j^{\prime}}}{\sqrt{2 j+1}} C_{m^{\prime} m}^{(j)} \Leftrightarrow m \longrightarrow \overbrace{j^{\prime}}^{j}=\frac{\delta_{j, j^{\prime}}}{\sqrt{2 j+1}} m \longrightarrow$

Coupling four angular momenta to a zero resultant will involve the $j m$-coefficients. The $j m$-coefficients corresponding to different coupling schemes are related by the $6 j$-symbol. The $6 j$-symbol is defined by ([41] p. 94)

$$
\begin{aligned}
\left\{\begin{array}{lll}
j_{1} & j_{2} & j_{3} \\
j_{4} & j_{5} & j_{6}
\end{array}\right\}: & \sum_{\text {all } m, m^{\prime}}\left(\begin{array}{ccc}
j_{1} & j_{2} & j_{3} \\
m_{1} & m_{2} & m_{3}
\end{array}\right)\left(\begin{array}{ccc}
j_{1} & j_{5} & j_{6} \\
m_{1}^{\prime} & m_{5} & m_{6}^{\prime}
\end{array}\right)\left(\begin{array}{ccc}
j_{4} & j_{2} & j_{6} \\
m_{4}^{\prime} & m_{2}^{\prime} & m_{6}
\end{array}\right)\left(\begin{array}{ccc}
j_{4} & j_{5} & j_{3} \\
m_{4} & m_{5}^{\prime} & m_{3}^{\prime}
\end{array}\right) \\
& \times C_{\left(j_{1}\right)}^{m_{1}^{\prime} m_{1}} C_{\left(j_{2}\right)}^{m_{2}^{\prime} m_{2}} C_{\left(j_{3}\right)}^{m_{3}^{\prime} m_{3}} C_{\left(j_{4}\right)}^{m_{4}^{\prime} m_{4}} C_{\left(j_{5}\right)}^{m_{5}^{\prime} m_{5}} C_{\left(j_{6}\right)}^{m_{6}^{\prime} m_{6}} .
\end{aligned}
$$

Graphically, we can express the $6 j$-symbol in Eq. (A.48) as

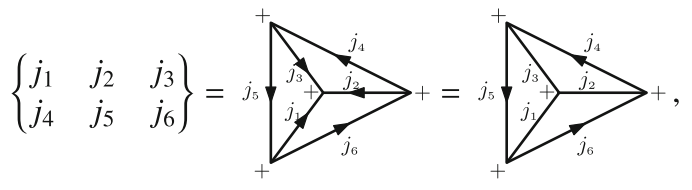


where in the last step we have used Eq. (A.44) to remove three arrows. Taking account of the definition of $6 j$-symbol in Eq. (A.48) and the fact that the $3 j$-symbol is normalized in Eq. (A.31), we can easily show the following identity (see [41], p. 95):

$\sum_{m_{4}, m_{5}, m_{6}, m_{4}^{\prime}, m_{5}^{\prime}, m_{6}^{\prime}}\left(\begin{array}{ccc}j_{1} & j_{5} & j_{6} \\ m_{1} & m_{5} & m_{6}^{\prime}\end{array}\right)\left(\begin{array}{ccc}j_{4} & j_{2} & j_{6} \\ m_{4}^{\prime} & m_{2} & m_{6}\end{array}\right)\left(\begin{array}{ccc}j_{4} & j_{5} & j_{3} \\ m_{4} & m_{5}^{\prime} & m_{3}\end{array}\right) C_{\left(j_{4}\right)}^{m_{4}^{\prime} m_{4}} C_{\left(j_{5}\right)}^{m_{5}^{\prime} m_{5}} C_{\left(j_{6}\right)}^{m_{6}^{\prime} m_{6}}=\left(\begin{array}{ccc}j_{1} & j_{2} & j_{3} \\ m_{1} & m_{2} & m_{3}\end{array}\right)\left\{\begin{array}{lll}j_{1} & j_{2} & j_{3} \\ j_{4} & j_{5} & j_{6}\end{array}\right\}$.

Hence we have the following graphical identity:

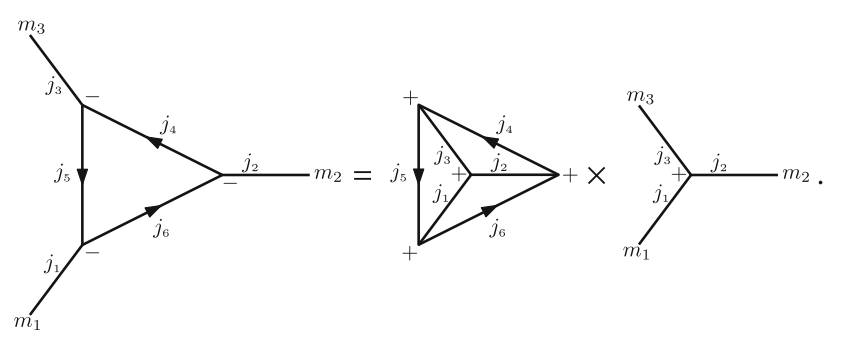

The following algebraic relation between two different coupling schemes:

$$
\sum_{m_{5}, m_{5}^{\prime}}\left(\begin{array}{ccc}
j_{1} & j_{3} & j_{5} \\
m_{1} & m_{3} & m_{5}
\end{array}\right) C_{\left(j_{5}\right)}^{m_{5} m_{5}^{\prime}}\left(\begin{array}{ccc}
j_{5} & j_{2} & j_{4} \\
m_{5}^{\prime} & m_{2} & m_{4}
\end{array}\right)=\sum_{j_{6}, m_{6}, m_{6}^{\prime}}\left(2 j_{6}+1\right)(-1)^{j_{2}+j_{3}+j_{5}+j_{6}}\left\{\begin{array}{lll}
j_{1} & j_{2} & j_{6} \\
j_{4} & j_{3} & j_{5}
\end{array}\right\}\left(\begin{array}{ccc}
j_{1} & j_{2} & j_{6} \\
m_{1} & m_{2} & m_{6}
\end{array}\right) C_{\left(j_{6}\right)}^{m_{6} m_{6}^{\prime}}\left(\begin{array}{ccc}
j_{6} & j_{3} & j_{4} \\
m_{6}^{\prime} & m_{3} & m_{4}
\end{array}\right)
$$

corresponds to the following rule of transforming graphs:

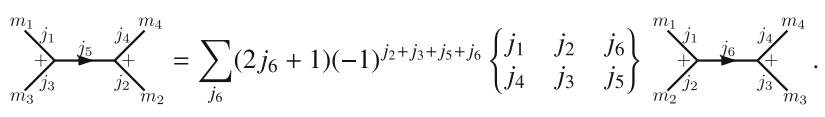

Using Eq. (A.33) and the symmetric properties of $6 j$-symbol, from Eq. (A.53), we can get

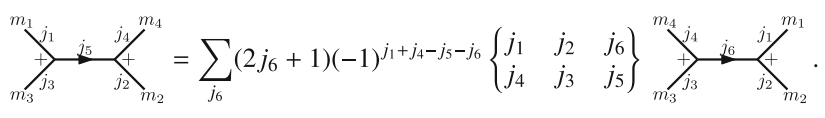

Similarly, using Eqs. (A.33) and (A.54), we have

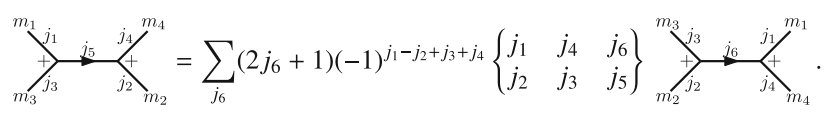

Note that Brink's graphical representation in [31] does not involve how to represent graphically the matrix element of the representation of $S U(2)$. Here, we will extend the Brink representation and propose a graphical representation for the unitary irreducible representation $\pi_{j}$ of $S U(2)$. The matrix element $\left[\pi_{j}(g)\right]_{n}^{m}$ is denoted by a blue line with a hollow arrow (triangle) in it,

$\left[\pi_{j}(g)\right]_{n}^{m}=: m \longrightarrow$

The orientation of the arrow is from its row index $m$ to its column index $n$. The matrix element $\left[\pi_{j}\left(g^{-1}\right)\right]_{m}^{n}$ in Eq. (A.12) can be presented by

$\left[\pi_{j}\left(g^{-1}\right)\right]_{m}^{n}={ }_{n} \longrightarrow \mathrm{g}^{j} \mathrm{C}_{m}={ }_{n} \longrightarrow \mathrm{j} \longrightarrow$ 
Up to now, we have expressed the quantum states [the spin-network states in (2.13)], and the two elementary operators (the holonomy and flux operators in (2.17) and (2.19)) of LQG in the graphical form. The Clebsch-Gordan series in (A.22) can be represented by

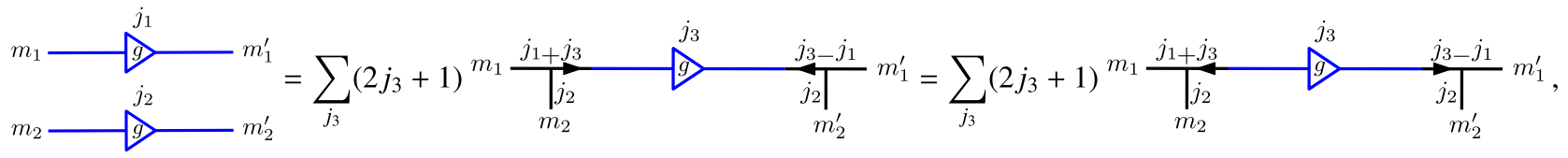

where, in the second step, we used (A.43) to flip the orientations of two arrows. Using Eqs. (A.57) and (A.58), we have

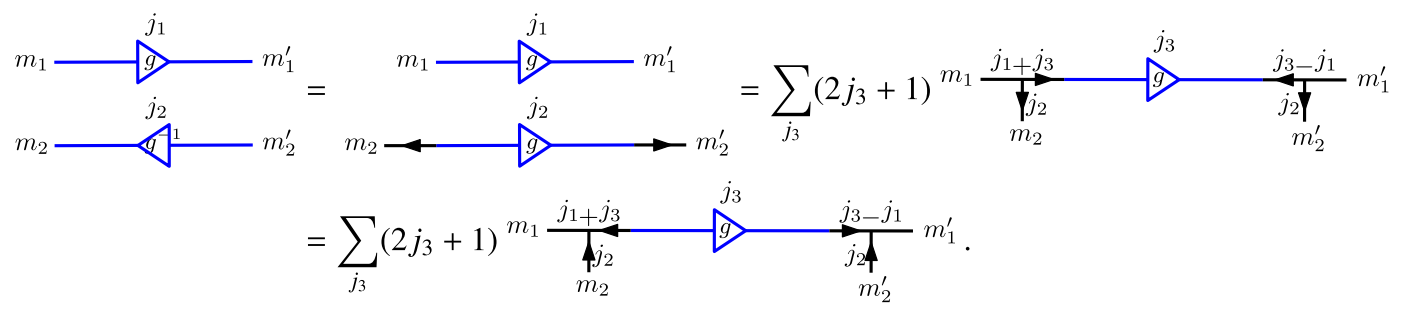

\section{Appendix B Proofs for some identities}

B.1 Proofs of algebraic identities in Eqs. (3.7) and (4.8)

By the definition of the matrix element

$\left[\pi_{j}\left(\tau_{i}\right)\right]_{m}^{m^{\prime}}:=\left.\frac{\mathrm{d}}{\mathrm{d} t}\right|_{t=0}\left[\pi_{j}\left(e^{t \tau_{i}}\right)\right]_{m}^{m^{\prime}}$,

we get

$\left[\pi_{j}\left(\tau_{1}\right)\right]^{m^{\prime}}{ }_{m}=-\frac{i}{2} \sqrt{j(j+1)-m^{\prime}\left(m^{\prime}+1\right)} \delta_{m^{\prime}, m-1}-\frac{i}{2} \sqrt{j(j+1)-m^{\prime}\left(m^{\prime}-1\right)} \delta_{m^{\prime}, m+1}$,

$\left[\pi_{j}\left(\tau_{2}\right)\right]^{m^{\prime}}{ }_{m}=\frac{1}{2} \sqrt{j(j+1)-m^{\prime}\left(m^{\prime}+1\right)} \delta_{m^{\prime}, m-1}-\frac{1}{2} \sqrt{j(j+1)-m^{\prime}\left(m^{\prime}-1\right)} \delta_{m^{\prime}, m+1}$,

$\left[\pi_{j}\left(\tau_{3}\right)\right]^{m^{\prime}}{ }_{m}=-i m^{\prime} \delta_{m^{\prime}, m}$.

Hence the matrix elements of $\tau_{\mu}(\mu=0, \pm 1)$ defined in Eq. (3.6) read

$$
\begin{aligned}
& {\left[\pi_{j}\left(\tau_{0}\right)\right]^{m^{\prime}}{ }_{m}=\left[\pi_{j}\left(\tau_{3}\right)\right]^{m^{\prime}}{ }_{m}=-i m^{\prime} \delta_{m^{\prime}, m},} \\
& {\left[\pi_{j}\left(\tau_{+1}\right)\right]_{m}^{m^{\prime}}=-\frac{1}{\sqrt{2}}\left(\left[\pi_{j}\left(\tau_{1}\right)\right]^{m^{\prime}}{ }_{m}+i\left[\pi_{j}\left(\tau_{2}\right)\right]^{m^{\prime}}{ }_{m}\right)=+i \frac{\sqrt{2}}{2} \sqrt{j(j+1)-m^{\prime}\left(m^{\prime}-1\right)} \delta_{m^{\prime}, m+1},} \\
& {\left[\pi_{j}\left(\tau_{-1}\right)\right]_{m}^{m^{\prime}}=+\frac{1}{\sqrt{2}}\left(\left[\pi_{j}\left(\tau_{1}\right)\right]_{m}^{m^{\prime}}-i\left[\pi_{j}\left(\tau_{2}\right)\right]^{m^{\prime}}{ }_{m}\right)=-i \frac{\sqrt{2}}{2} \sqrt{j(j+1)-m^{\prime}\left(m^{\prime}+1\right)} \delta_{m^{\prime}, m-1} .}
\end{aligned}
$$

Taking account of the specialized formulas for the CGCs,

$$
\left\langle j m 10 \mid j m^{\prime}\right\rangle=\frac{m^{\prime}}{\sqrt{j(j+1)}} \delta_{m^{\prime}, m}=-\sqrt{2 j+1}\left(\begin{array}{ccc}
j & 1 & j \\
m & 0 & m^{\prime \prime}
\end{array}\right) C_{(j)}^{m^{\prime \prime} m^{\prime}},
$$


$\left\langle j m 11 \mid j m^{\prime}\right\rangle=-\sqrt{\frac{j(j+1)-m^{\prime}\left(m^{\prime}-1\right)}{2 j(j+1)}} \delta_{m^{\prime}, m+1}=-\sqrt{2 j+1}\left(\begin{array}{ccc}j & 1 & j \\ m & 1 & m^{\prime \prime}\end{array}\right) C_{(j)}^{m^{\prime \prime} m^{\prime}}$,
$\left\langle j m 1-1 \mid j m^{\prime}\right\rangle=+\sqrt{\frac{j(j+1)-m^{\prime}\left(m^{\prime}+1\right)}{2 j(j+1)}} \delta_{m^{\prime}, m-1}=-\sqrt{2 j+1}\left(\begin{array}{ccc}j & 1 & j \\ m & -1 & m^{\prime \prime}\end{array}\right) C_{(j)}^{m^{\prime \prime} m^{\prime}}$,

we get

$\left[\pi_{j}\left(\tau_{\mu}\right)\right]^{m^{\prime}}{ }_{m}=\frac{i}{2} \sqrt{2 j(2 j+1)(2 j+2)}\left(\begin{array}{ccc}j & 1 & j \\ m & \mu & m^{\prime \prime}\end{array}\right) C_{(j)}^{m^{\prime \prime} m^{\prime}}=\frac{i}{2} \sqrt{2 j(2 j+1)(2 j+2)}\left(\begin{array}{ccc}1 & j & j \\ \mu & m^{\prime \prime} & m\end{array}\right) C_{(j)}^{m^{\prime \prime} m^{\prime}}$.

By definition (3.6), we have

$\tau_{1}=-\frac{1}{\sqrt{2}}\left(\tau_{+1}-\tau_{-1}\right), \quad \tau_{2}=\frac{i}{\sqrt{2}}\left(\tau_{+1}+\tau_{-1}\right), \quad \tau_{3}=\tau_{0}$

Then

$$
\begin{aligned}
& {\left[\pi_{j_{I}}\left(\tau_{i}\right)\right]_{m_{I}}^{m_{I}^{\prime}}\left[\pi_{j_{J}}\left(\tau_{i}\right)\right]^{m_{J}^{\prime}}{ }_{m_{J}}=-\left[\pi_{j_{I}}\left(\tau_{+1}\right)\right]^{m_{I}^{\prime}{ }_{m_{I}}}\left[\pi_{j_{J}}\left(\tau_{-1}\right)\right]^{m_{J}^{\prime}}{ }_{m_{J}}-\left[\pi_{j_{I}}\left(\tau_{-1}\right)\right]^{m_{I}^{\prime}{ }_{m_{I}}}\left[\pi_{j_{J}}\left(\tau_{+1}\right)\right]^{m_{J}^{\prime}}{ }_{m_{J}}} \\
& +\left[\pi_{j_{I}}\left(\tau_{0}\right)\right]^{m_{I}^{\prime} m_{I}}\left[\pi_{j_{J}}\left(\tau_{0}\right)\right]^{m_{J}^{\prime}}{ }_{m_{J}} \\
& =-\left[\pi_{j_{I}}\left(\tau_{\mu}\right)\right]_{m_{I}}^{m_{I}^{\prime}} C_{(1)}^{\mu^{\prime} \mu}\left[\pi_{j_{J}}\left(\tau_{\mu^{\prime}}\right)\right]^{m_{J}^{\prime}}{ }_{m_{J}} \text {. }
\end{aligned}
$$

B.2 Proofs of graphical identities in Eqs. (4.14), (4.15), (4.16) and (4.17)

Equation (4.14) can be proved by

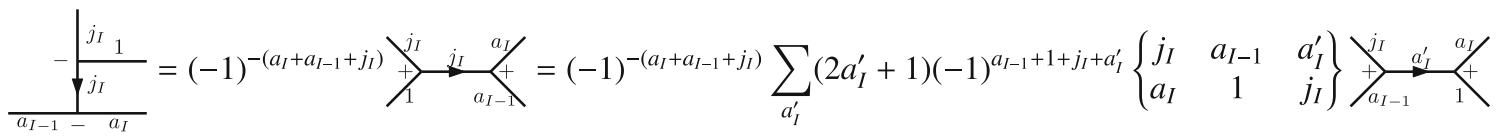

$$
\begin{aligned}
& =(-1)^{-\left(a_{I}+a_{I-1}+j_{I}\right)} \sum_{a_{I}^{\prime}}\left(2 a_{I}^{\prime}+1\right)(-1)^{a_{I-1}+1+j_{I}+a_{I}^{\prime}}(-1)^{a_{I}+a_{I}^{\prime}+1}(-1)^{j_{I}+a_{I-1}+a_{I}^{\prime}}\left\{\begin{array}{ccc}
j_{I} & a_{I-1} & a_{I}^{\prime} \\
a_{I} & 1 & j_{I}
\end{array}\right\} \sum_{a_{I-1}}^{j_{I}}{\stackrel{a}{a_{I}^{\prime}}}_{1}^{a_{I}} \\
& =\left.\sum_{a_{I}^{\prime}}\left(2 a_{I}^{\prime}+1\right)(-1)^{a_{I-1}-a_{I}^{\prime}+j_{I}}\left\{\begin{array}{ccc}
a_{I-1} & j_{I} & a_{I} \\
1 & a_{I}^{\prime} & j_{I}
\end{array}\right\} \stackrel{b_{I I}}{a_{I-1}-a_{I}^{\prime}}\right|_{1} ^{a_{I}},
\end{aligned}
$$

where Eq. (A.33) was used in the first and third steps, (A.53) was used in the second step, and we used the fact $(-1)^{-4 a_{1}^{\prime}}=1$ and the symmetric properties of the $6 j$-symbol in the last step.

Equation (4.15) can be shown by

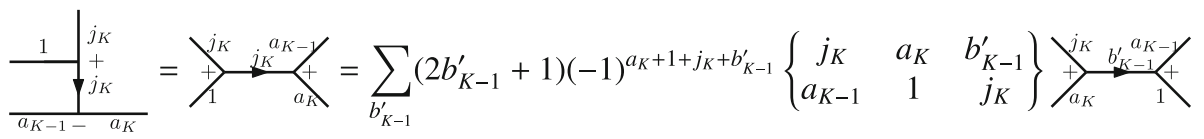

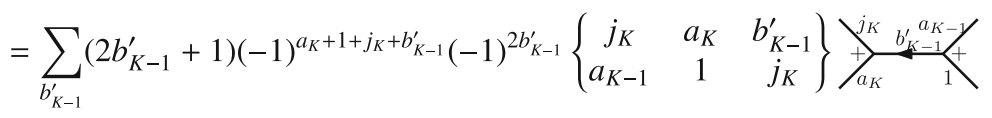

$$
\begin{aligned}
& =\sum_{b_{K-1}^{\prime}}\left(2 b_{K-1}^{\prime}+1\right)(-1)^{a_{K}-b_{K-1}^{\prime}+j_{K}+1}\left\{\begin{array}{ccc}
a_{K} & j_{K} & a_{K-1} \\
1 & b_{K-1}^{\prime} & j_{K}
\end{array}\right\} \underset{\left.a_{K-1}\right|_{1} ^{b_{K-1}^{\prime}-a_{K}}}{-}
\end{aligned}
$$

where Eq. (A.53) was used in the second step, and in the last step we used Eq. (A.43), the fact $(-1)^{-4 b_{K-1}^{\prime}}=1$, and the symmetric properties of the $6 j$-symbol. 
Equation (4.16) can be proved by

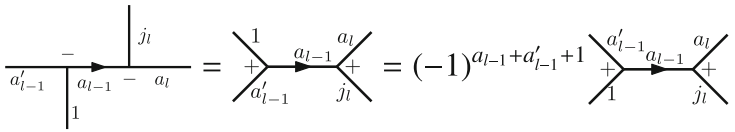

$$
\begin{aligned}
& =(-1)^{a_{l-1}+a_{l-1}^{\prime}+1} \sum_{a_{l}^{\prime}}\left(2 a_{l}^{\prime}+1\right)(-1)^{j_{l}+1+a_{l-1}+a_{l}^{\prime}}\left\{\begin{array}{ccc}
a_{l-1}^{\prime} & j_{l} & a_{l}^{\prime} \\
a_{l} & 1 & a_{l-1}
\end{array}\right\} \overbrace{j_{l}}^{a_{l-1}^{\prime}} \underbrace{a_{l}^{\prime}}_{1} a_{+}^{a_{l}}+ \\
& =\left.(-1)^{a_{l-1}+a_{l-1}^{\prime}+1} \sum_{a_{l}^{\prime}}\left(2 a_{l}^{\prime}+1\right)(-1)^{j_{l}+1+a_{l-1}+a_{l}^{\prime}}(-1)^{-\left(a_{l}+a_{l}^{\prime}+1\right)}\left\{\begin{array}{ccc}
a_{l-1}^{\prime} & j_{l} & a_{l}^{\prime} \\
a_{l} & 1 & a_{l-1}
\end{array}\right\} \frac{\left.\right|_{a_{l-1}^{\prime}-} ^{j_{l}}}{-a_{l}^{\prime}}\right|_{1} ^{a_{l}} \\
& =\sum_{a_{l}^{\prime}}\left(2 a_{l}^{\prime}+1\right)(-1)^{a_{l-1}^{\prime}+a_{l-1}+1}(-1)^{a_{l-1}-a_{l}+j_{l}}\left\{\begin{array}{ccc}
j_{l} & a_{l-1}^{\prime} & a_{l}^{\prime} \\
1 & a_{l} & a_{l-1}
\end{array}\right\} \frac{\mid j_{l-1}-\vec{a}_{l}^{\prime}}{a_{l}^{\prime}},
\end{aligned}
$$

where Eq. (A.33) was used in the second and fourth steps, (A.53) was used in the third step, and in the last step we used the symmetric properties of the $6 j$-symbol and the exponents were simplified.

Equation (4.17) can be proved by

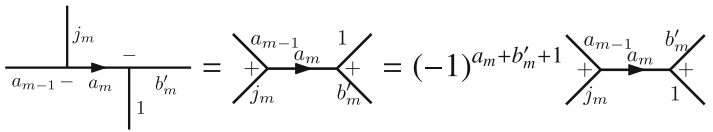

$$
\begin{aligned}
& =(-1)^{a_{m}+b_{m}^{\prime}+1} \sum_{b_{m-1}^{\prime}}\left(2 b_{m-1}^{\prime}+1\right)(-1)^{1+j_{m}+a_{m}+b_{m-1}^{\prime}}\left\{\begin{array}{ccc}
a_{m-1} & 1 & b_{m-1}^{\prime} \\
b_{m}^{\prime} & j_{m} & a_{m}
\end{array}\right\} \overbrace{1}^{a_{m-1}-1} \underbrace{b_{m}^{\prime}}_{b_{m-1}^{\prime}}
\end{aligned}
$$

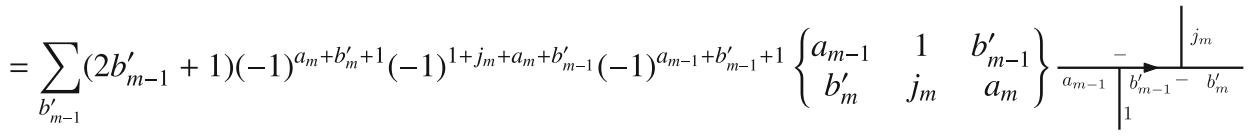

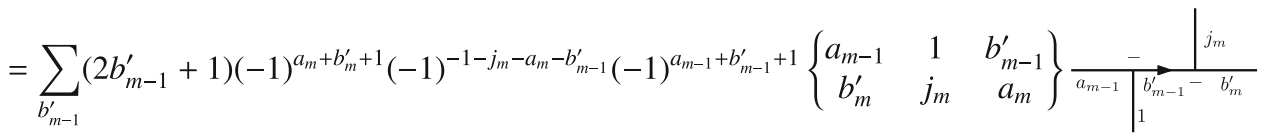

$$
\begin{aligned}
& =\sum_{b_{m-1}^{\prime}}\left(2 b_{m-1}^{\prime}+1\right)(-1)^{b_{m-1}^{\prime}+a_{m-1}+1}(-1)^{b_{m}^{\prime}-b_{m-1}^{\prime}-j_{m}}\left\{\begin{array}{ccc}
j_{m} & b_{m-1}^{\prime} & b_{m}^{\prime} \\
1 & a_{m} & a_{m-1}
\end{array}\right\} \underset{a_{m-1}}{-\underset{b_{m-1}^{\prime}-b_{m}^{\prime}}{\mid}},
\end{aligned}
$$

where Eq. (A.33) was used in the second and fourth steps, (A.53) was used in the third step, and in the last step we used the symmetric properties of the $6 j$-symbol and the exponents were simplified.

B.3 Proofs of graphical identities in Eqs. (4.18) and (4.24)

Equation (4.18) can be proved by

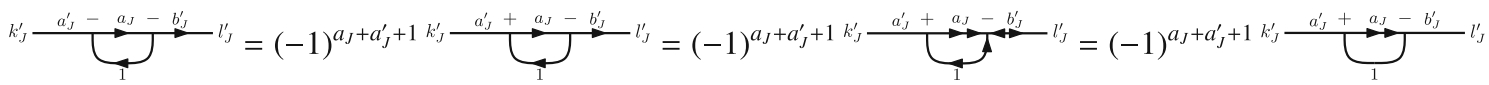

$$
\begin{aligned}
& =(-1)^{a_{J}+a_{J}^{\prime}+1}(-1)^{2 a_{J} k_{J}^{\prime}} \underbrace{b_{J}^{\prime}}_{a_{J}^{\prime}+a_{J}-b_{J}^{\prime}}=(-1)^{a_{J}^{\prime}-a_{J}+1} \frac{\delta_{a_{J}^{\prime}, b_{J}^{\prime}}}{2 a_{J}^{\prime}+1} k_{J}^{\prime}{\stackrel{a_{J}^{\prime}}{ }}_{l_{J}^{\prime}},
\end{aligned}
$$

where the identities in Eqs. (A.33), (A.44), (A.41), (A.42) and (A.45) were used from the first to last steps. 
Equation (4.24) can be proved by

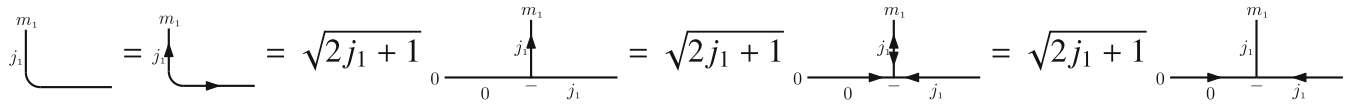

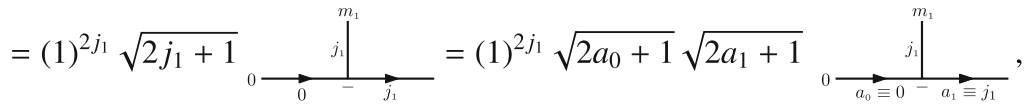

where the rules (A.41), (A.47), (A.44), (A.41) and (A.43) were used from the first to fifth steps, and in last step we denoted $a_{0} \equiv 0, a_{1} \equiv j_{1}$.

B.4 Proofs of graphical identities in Eqs. (5.21) and (5.22)

The graph on the left-hand side of (A.51) can be transformed to
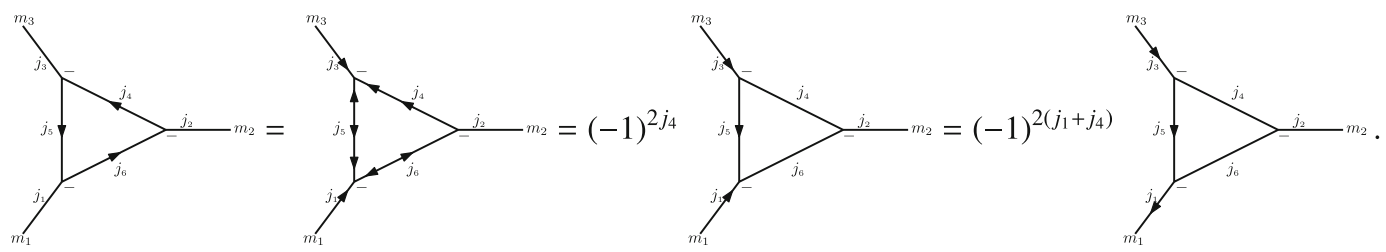

The first graph on the right-hand side of (A.51) represents the $6 j$-symbol, which can be transformed as
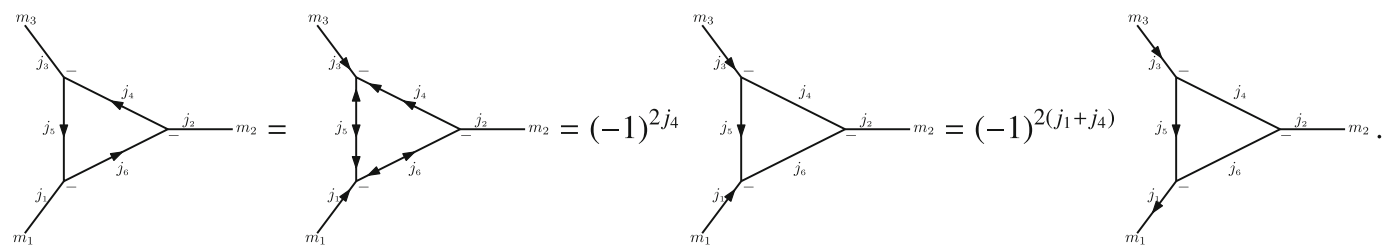

where we have used the rules (A.41)-(A.44) of transforming graphs. Hence Eq. (A.51) is equal to the following graphical identity:

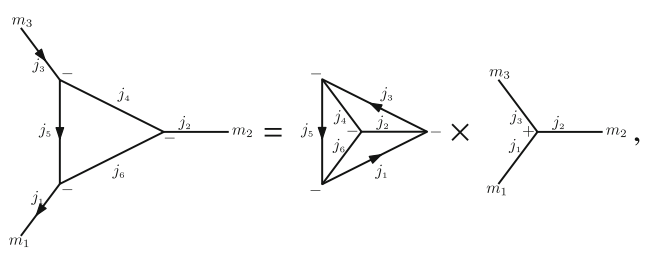

which implies

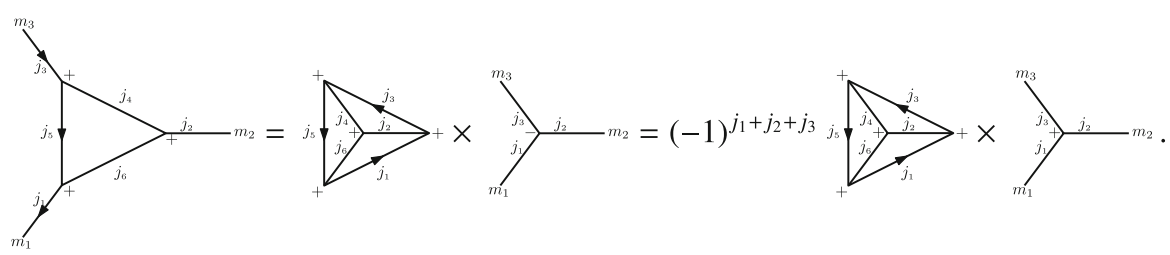


Graphically, Eq. (5.21) can be proved by

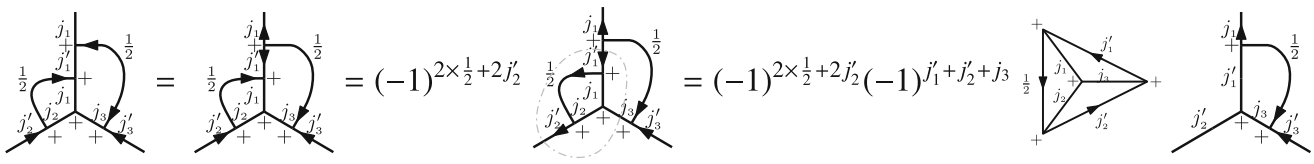

$$
\begin{aligned}
& =(-1)^{\frac{1}{2} \times 2+2 j_{2}^{\prime}}(-1)^{j_{1}^{\prime}+j_{2}^{\prime}+j_{3}}(-1)^{2 \times \frac{1}{2}} \\
& =(-1)^{\frac{1}{2} \times 2+2 j_{2}^{\prime}}(-1)^{j_{1}^{\prime}+j_{2}^{\prime}+j_{3}}(-1)^{2 \times \frac{1}{2}}(-1)^{j_{1}+j_{2}^{\prime}+j_{3}^{\prime}} \frac{1}{2} \\
& \left.=-(-1)^{j_{1}+j_{1}^{\prime}+\frac{1}{2}}(-1)^{j_{3}+j_{3}^{\prime}+\frac{1}{2}}\left\{\begin{array}{lll}
j_{1} & \frac{1}{2} & j_{1}^{\prime} \\
j_{2}^{\prime} & j_{3} & j_{2}
\end{array}\right\}\left\{\begin{array}{ccc}
j_{1} & \frac{1}{2} & j_{1}^{\prime} \\
j_{3} & j_{2}^{\prime} & j_{3}^{\prime}
\end{array}\right\} j_{2}^{j_{1}}\right\}_{j_{3}^{\prime}} \text {, }
\end{aligned}
$$

where we have used the rules (A.41)-(A.44), and (B.23) in the third and fifth steps, respectively. Similarly, Eq. (5.22) can be shown by

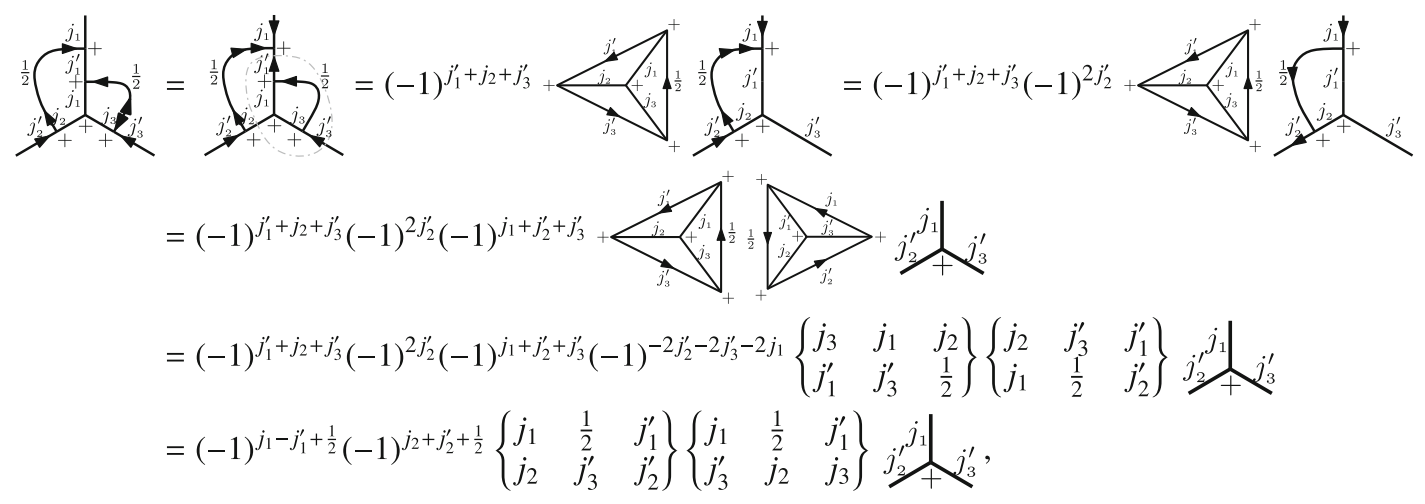

where we have used (B.23) in the second and fourth steps, and used the fact that the allowed triple $\left(j_{2}^{\prime}, j_{3}^{\prime}, j_{1}\right)$ satisfy the triangular condition in the fifth step.

B.5 Proofs of graphical identities in Eqs. (6.18) and (6.25)

The identity (6.18) can be proved by

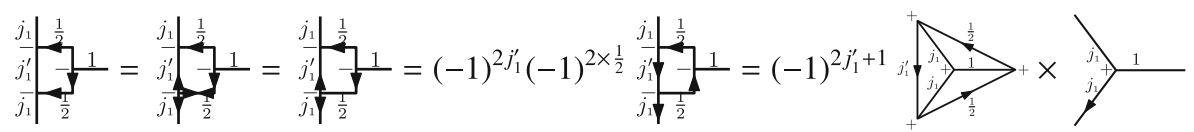

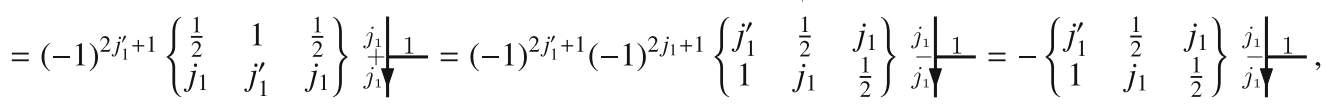

where we have used (A.51) in fourth step, and used the fact that $(-1)^{2 j_{1}^{\prime}+2 j_{1}+1}=1$ in the last step, since the allowed triple $\left(j_{1}^{\prime}, j_{1}, \frac{1}{2}\right)$ satisfy the triangular condition. 
Equation (6.25) can be obtained from

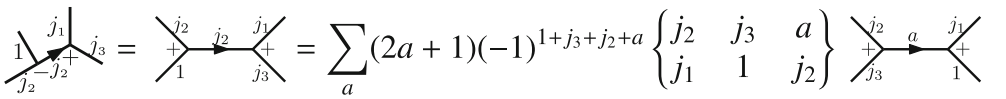

$$
\begin{aligned}
& =\sum_{a}(2 a+1)(-1)^{1+j_{3}+j_{2}+a}\left\{\begin{array}{ccc}
j_{2} & j_{3} & a \\
j_{1} & 1 & j_{2}
\end{array}\right\}(-1)^{j_{1}+a+1}(-1)^{2 a}>_{j_{3}}^{j_{2}} \underbrace{j_{1}}_{-a} \\
& \left.=(-1)^{j_{1}+j_{2}+j_{3}} \sum_{a}(2 a+1)\left\{\begin{array}{ccc}
j_{3} & j_{2} & a \\
1 & j_{1} & j_{2}
\end{array}\right\}, \begin{array}{c}
j_{1} \\
j_{2}
\end{array}\right\}_{j_{3}},
\end{aligned}
$$

where in the second step we have used the identity (A.53).

B.6 Proofs of algebraic identities in Eqs. (7.5) and (7.6)

To prove Eq. (7.5), we denote $\left[\pi_{j_{I}}\left(\tau_{i}\right)\right]^{n_{I}}{ }_{m_{I}}\left[\pi_{j_{J}}\left(\tau_{j}\right)\right]^{n_{J}{ }_{m_{J}}}\left[\pi_{j_{K}}\left(\tau_{k}\right)\right]^{n_{K}}{ }_{m_{K}}$ by $\left[\tau_{i} \tau_{j} \tau_{k}\right]_{n_{I} m_{I} n_{J} m_{J} n_{K} m_{K}}$. Taking account of (B.12), we have

$\tau_{1} \tau_{2}-\tau_{2} \tau_{1}=-\frac{1}{\sqrt{2}} \frac{i}{\sqrt{2}}\left[\left(\tau_{+1}-\tau_{-1}\right)\left(\tau_{+1}+\tau_{-1}\right)-\left(\tau_{+1}+\tau_{-1}\right)\left(\tau_{+1}-\tau_{-1}\right)\right]=-i\left(\tau_{+1} \tau_{-1}-\tau_{-1} \tau_{+1}\right)$

Then we have

$$
\begin{aligned}
\epsilon_{i j k} & {\left[\pi_{j_{I}}\left(\tau_{i}\right)\right]_{m_{I}}^{n_{I}}\left[\pi_{j_{J}}\left(\tau_{j}\right)\right]_{m_{J}}^{n_{J}}\left[\pi_{j_{K}}\left(\tau_{k}\right)\right]^{n_{K}}{ }_{m_{K}}=+\epsilon_{123}\left[\tau_{1} \tau_{2} \tau_{3}\right]_{n_{I} m_{I} n_{J} m_{J} n_{K} m_{K}}+\epsilon_{132}\left[\tau_{1} \tau_{3} \tau_{2}\right]_{n_{I} m_{I} n_{J} m_{J} n_{K} m_{K}} } \\
& +\epsilon_{213}\left[\tau_{2} \tau_{1} \tau_{3}\right]_{n_{I} m_{I} n_{J} m_{J} n_{K} m_{K}}+\epsilon_{312}\left[\tau_{3} \tau_{1} \tau_{2}\right]_{n_{I} m_{I} n_{J} m_{J} n_{K} m_{K}} \\
& +\epsilon_{231}\left[\tau_{2} \tau_{3} \tau_{1}\right]_{n_{I} m_{I} n_{J} m_{J} n_{K} m_{K}}+\epsilon_{321}\left[\tau_{3} \tau_{2} \tau_{1}\right]_{n_{I} m_{I} n_{J} m_{J} n_{K} m_{K}} \\
= & {\left[\tau_{1} \tau_{2}-\tau_{2} \tau_{1}\right]_{n_{I} m_{I} n_{J} m_{J}}\left[\tau_{3}\right]_{n_{K} m_{K}}+\left[\tau_{3}\right]_{n_{I} m_{I}}\left[\tau_{1} \tau_{2}-\tau_{2} \tau_{1}\right]_{n_{J} m_{J} n_{K} m_{K}} } \\
& -\left[\tau_{1} \tau_{3} \tau_{2}-\tau_{2} \tau_{3} \tau_{1}\right]_{n_{I} m_{I} n_{J} m_{J} n_{K} m_{K}} \\
= & -i\left[\tau_{+1} \tau_{-1}-\tau_{-1} \tau_{+1}\right]_{n_{I} m_{I} n_{J} m_{J}}\left[\tau_{0}\right]_{n_{K} m_{K}}-i\left[\tau_{0}\right]_{n_{I} m_{I}}\left[\tau_{+1} \tau_{-1}\right. \\
& \left.-\tau_{-1} \tau_{+1}\right]_{n_{J} m_{J} n_{K} m_{K}}-(-i)\left[\tau_{+1} \tau_{0} \tau_{-1}-\tau_{-1} \tau_{0} \tau_{+1}\right]_{n_{I} m_{I} n_{J} m_{J} n_{K} m_{K}} \\
= & -i\left[\epsilon_{+1-10} \tau_{+1} \tau_{-1} \tau_{0}+\epsilon_{-1+10} \tau_{-1} \tau_{+1} \tau_{0}+\epsilon_{0+1-1} \tau_{0} \tau_{+1} \tau_{-1}\right. \\
& \left.+\epsilon_{0-1+1} \tau_{0} \tau_{-1} \tau_{+1}+\epsilon_{-10+1} \tau_{-1} \tau_{0} \tau_{+1}+\epsilon_{+10-1} \tau_{+1} \tau_{0} \tau_{-1}\right]_{n_{I} m_{I} n_{J} m_{J} n_{K} m_{K}} \\
= & -i \epsilon_{\mu v \rho}\left[\tau_{\mu} \tau_{\nu} \tau_{\rho}\right]_{n_{I} m_{I} n_{J} m_{J} n_{K} m_{K}} \\
= & -i \epsilon_{\mu \nu \rho}\left[\pi_{j_{I}}\left(\tau_{\mu}\right)\right]_{{ }_{m_{I}}}^{n_{I}}\left[\pi_{j_{J}}\left(\tau_{v}\right)\right]_{m_{J}}^{n_{J}}\left[\pi_{j_{K}}\left(\tau_{\rho}\right)\right]^{n_{K}}{ }_{m_{K}},
\end{aligned}
$$

where $\epsilon_{\mu \nu \rho}$ is the Levi-Cività symbol defined by $\epsilon_{-10+1}=1$.

To show Eq. (7.6), notice that

$$
\left(\begin{array}{ccc}
1 & 1 & 1 \\
-1 & 0 & 1
\end{array}\right)=\frac{1}{\sqrt{6}}=\frac{1}{\sqrt{6}} \epsilon_{-10+1}
$$

Recalling the symmetric property of the $3 j$-symbol in Appendix A.2, an even permutation of the columns leaves the numerical value unchanged, while an odd permutation will lead to a factor $(-1)^{1+1+1}=-1$ for the $3 j$-symbol in Eq. (A.33). These symmetries of the $3 j$-symbol are the same as those of $\epsilon_{\mu \nu \rho}$. Hence we have $\epsilon_{\mu \nu \rho}=\sqrt{6}\left(\begin{array}{lll}1 & 1 & 1 \\ \mu & \nu & \rho\end{array}\right)$. 


\section{Appendix C The diagonalization of the volume operator in 2-d intertwiner space}

We denote

$\left|\alpha_{1}\right\rangle \equiv\left|1 / 2, j_{1}^{\prime}, j_{2}, j_{3} ; a_{2}=j_{1}^{\prime}-1 / 2, a_{3}=j_{3}, J=0\right\rangle \equiv \sqrt{2 a_{2}+1} \sqrt{2 a_{3}+1} \underbrace{\left.\left.\stackrel{\frac{1}{2}}{j_{1}^{\prime}}\right|_{J=0} ^{j_{2}}\right|_{j_{3}}}_{a_{2}=j_{1}^{\prime}-\frac{1}{2} a_{3}=j_{3}-J=0}$,

$\left|\alpha_{2}\right\rangle \equiv\left|1 / 2, j_{1}^{\prime}, j_{2}, j_{3} ; a_{2}=j_{1}^{\prime}+1 / 2, a_{3}=j_{3}, J=0\right\rangle \equiv \sqrt{2 a_{2}+1} \sqrt{2 a_{3}+1} \underbrace{\left.\left.\right|_{j_{1}^{\prime}}\right|_{j_{2}} ^{j_{3}}}_{a_{a_{2}=j_{1}^{\prime}+\frac{-}{2}} a_{3}=j_{3}-j_{J=0}}$.

The matrix of the operator $i \hat{q}_{j_{1}^{\prime} j_{2} j_{3}}$ in the above two states reads

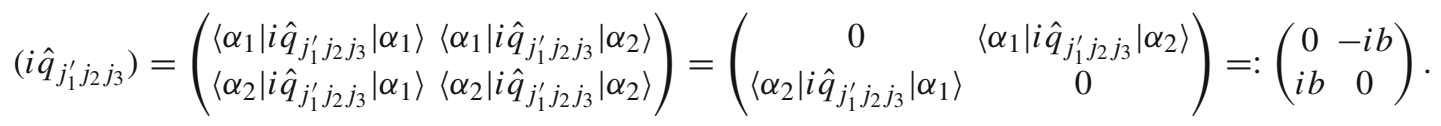

The eigenvalues and corresponding (normalized) eigenvectors of $i \hat{q}_{j_{1}^{\prime} j_{2} j_{3}}$ are given by

$\lambda_{1}=-b \quad \rightarrow \quad\left|e_{1}\right\rangle=\frac{1}{\sqrt{2}}\left(\begin{array}{l}i \\ 1\end{array}\right), \lambda_{2}=b \quad \rightarrow \quad\left|e_{2}\right\rangle=\frac{1}{\sqrt{2}}\left(\begin{array}{c}-i \\ 1\end{array}\right)$.

Hence we obtain

$\sqrt{\left|i \hat{q}_{j_{1}^{\prime} j_{2} j_{3}}\right|}\left|\alpha_{i}\right\rangle=\sum_{k=1}^{2}\left|e_{k}\right\rangle\left\langle e_{k}\left|\sqrt{\left|i \hat{q}_{j_{1}^{\prime} j_{2} j_{3}}\right|}\right| \alpha_{i}\right\rangle=\sum_{k=1}^{2} \sqrt{\left|\lambda_{k}\right|}\left|e_{k}\right\rangle\left\langle e_{k} \mid \alpha_{i}\right\rangle=\sqrt{|b|}\left|\alpha_{i}\right\rangle, \quad i=1,2$.

Now we derive the value of $|b|$. Using the matrix elements of $\left\langle\vec{a}^{\prime}\left|\hat{q}_{234}\right| \vec{a}\right\rangle$ in Eq. (4.36), we have

$$
\begin{aligned}
\left\langle\alpha_{1}\left|\hat{q}_{j_{1}^{\prime} j_{2} j_{3}}\right| \alpha_{2}\right\rangle \equiv & \left\langle a_{2}-1=j_{1}^{\prime}-\frac{1}{2}\left|\hat{q}_{j_{1}^{\prime} j_{2} j_{3}}\right| a_{2}=j_{1}^{\prime}+\frac{1}{2}\right\rangle \\
= & -\frac{1}{4}(-1)^{\frac{1}{2}+j_{1}^{\prime}+j_{3}}(-1)^{j_{1}^{\prime}+\frac{1}{2}-\left(j_{1}^{\prime}-\frac{1}{2}\right)} X\left(j_{1}^{\prime}, j_{2}\right)^{\frac{1}{2}} X\left(j_{2}, j_{3}\right)^{\frac{1}{2}} \\
& \times \sqrt{2 j_{1}^{\prime}\left(2 j_{1}^{\prime}+2\right)}\left(2 j_{3}+1\right)\left\{\begin{array}{ccc}
\frac{1}{2} & j_{1}^{\prime} & j_{1}^{\prime}+\frac{1}{2} \\
1 & j_{1}^{\prime}-\frac{1}{2} & j_{1}^{\prime}
\end{array}\right\}\left\{\begin{array}{lll}
0 & j_{3} & j_{3} \\
1 & j_{3} & j_{3}
\end{array}\right\} \\
& \times\left[(-1)^{j_{1}^{\prime}-\frac{1}{2}+j_{3}}\left\{\begin{array}{ccc}
j_{3} & j_{2} & j_{1}^{\prime}+\frac{1}{2} \\
1 & j_{1}^{\prime}-\frac{1}{2} & j_{2}
\end{array}\right\}\left\{\begin{array}{rrr}
j_{1}^{\prime}-\frac{1}{2} & j_{2} & j_{3} \\
1 & j_{3} & j_{2}
\end{array}\right\}\right. \\
& \left.\times-(-1)^{j_{1}^{\prime}+\frac{1}{2}+j_{3}}\left\{\begin{array}{ccc}
j_{3} & j_{2} & j_{1}^{\prime}+\frac{1}{2} \\
1 & j_{1}^{\prime}-\frac{1}{2} & j_{2}
\end{array}\right\}\left\{\begin{array}{rrr}
j_{1}^{\prime}+\frac{1}{2} & j_{2} & j_{3} \\
1 & j_{3} & j_{2}
\end{array}\right\}\right] \\
= & {\left[\left(j_{1}^{\prime}+j_{2}+j_{3}+\frac{3}{2}\right)\left(j_{1}^{\prime}+j_{2}-j_{3}+\frac{1}{2}\right)\left(j_{1}^{\prime}-j_{2}+j_{3}+\frac{1}{2}\right)\left(-j_{1}^{\prime}+j_{2}+j_{3}+\frac{1}{2}\right)\right]^{\frac{1}{2}} . }
\end{aligned}
$$

Therefore, we have

$$
\begin{aligned}
\hat{V}\left|\alpha_{i}\right\rangle=\frac{\ell_{\mathrm{p}}^{3} \beta^{3 / 2}}{4 \sqrt{2}} \sqrt{\left|i \hat{q}_{j_{1}^{\prime} j_{2} j_{3}}\right|}\left|\alpha_{i}\right\rangle=\frac{\ell_{\mathrm{p}}^{3} \beta^{3 / 2}}{4 \sqrt{2}} \sqrt{|b|}\left|\alpha_{i}\right\rangle= & \frac{\ell_{\mathrm{p}}^{3} \beta^{3 / 2}}{4 \sqrt{2}}\left[\left(j_{1}^{\prime}+j_{2}+j_{3}+\frac{3}{2}\right)\left(j_{1}^{\prime}+j_{2}-j_{3}+\frac{1}{2}\right)\right. \\
& \left.\times\left(j_{1}^{\prime}-j_{2}+j_{3}+\frac{1}{2}\right)\left(-j_{1}^{\prime}+j_{2}+j_{3}+\frac{1}{2}\right)\right]^{\frac{1}{4}}\left|\alpha_{i}\right\rangle \\
& \equiv V\left(1 / 2, j_{1}^{\prime}, j_{2}, j_{3} ; a_{2}=j_{1}^{\prime}+1 / 2, a_{3}=j_{3}\right)\left|\alpha_{i}\right\rangle, \quad i=1,2,
\end{aligned}
$$

which reveals that the volume operator is diagonal in the 2-dimensional intertwiner space. 


\section{References}

1. A. Ashtekar, J. Lewandowski, Background independent quantum gravity: a status report. Class. Quant. Grav. 21, R53 (2004). doi:10.1088/ 0264-9381/21/15/R01. arXiv:gr-qc/0404018 [gr-qc]

2. M. Han, Y. Ma, W. Huang, Fundamental structure of loop quantum gravity. Int. J. Mod. Phys. D. 16, 1397-1474 (2007). doi:10.1142/ S0218271807010894. arXiv:gr-qc/0509064 [gr-qc]

3. C. Rovelli, Quantum Gravity (Cambridge University Press, Cambridge, 2004)

4. T. Thiemann, Modern Canonical Quantum General Relativity (Cambridge University Press, Cambridge, 2007)

5. T. Thiemann, A length operator for canonical quantum gravity. J. Math. Phys. 39, 3372-3392 (1998). doi:10.1063/1.532445. arXiv:gr-qc/9606092 [gr-qc]

6. E. Bianchi, The length operator in loop quantum gravity. Nucl. Phys. B. 807, 591-624 (2009). doi:10.1016/j.nuclphysb.2008.08.013. arXiv:0806.4710 [gr-qc]

7. Y. Ma, C. Soo, J. Yang, New length operator for loop quantum gravity. Phys. Rev. D 81, 124026 (2010). doi:10.1103/PhysRevD.81.124026. arXiv:1004.1063 [gr-qc]

8. C. Rovelli, L. Smolin, Discreteness of area and volume in quantum gravity. Nucl. Phys. B. 442, 593-622 (1995). doi:10.1016/ 0550-3213(95)00150-Q. arXiv:gr-qc/9411005 [gr-qc]

9. A. Ashtekar, J. Lewandowski, Quantum theory of geometry: I. Area operators. Class. Quant. Grav. 14, A55-A82 (1997). doi:10.1088/ 0264-9381/14/1A/006. arXiv:gr-qc/9602046 [gr-qc]

10. A. Ashtekar, J. Lewandowski, Quantum theory of geometry: II. Volume operators. Adv. Theor. Math. Phys 1, $388-429$ (1997). arXiv:gr-qc/9711031 [gr-qc]

11. T. Thiemann, Closed formula for the matrix elements of the volume operator in canonical quantum gravity. J. Math. Phys. 39, 3347-3371 (1998). doi:10.1063/1.532259. arXiv:gr-qc/9606091 [gr-qc]

12. K. Noui, A. Perez, Three-dimensional loop quantum gravity: Physical scalar product and spin foam models. Class. Quant. Grav. 22, 1739-1762 (2005). doi:10.1088/0264-9381/22/9/017. arXiv:gr-qc/0402110 [gr-qc]

13. E. Alesci, C. Rovelli, Regularization of the Hamiltonian constraint compatible with the spinfoam dynamics. Phys. Rev. D 82, 044007 (2010), doi:10.1103/PhysRevD.82.044007. arXiv:1005.0817 [gr-qc]

14. E. Alesci, T. Thiemann, A. Zipfel, Linking covariant and canonical LQG: new solutions to the Euclidean scalar constraint. Phys. Rev. D 86, 024017 (2012). doi:10.1103/PhysRevD.86.024017. arXiv:1109.1290 [gr-qc]

15. T. Thiemann, A. Zipfel, Linking covariant and canonical LQG II: spin foam projector. Class. Quant. Grav. 31, 125008 (2014). doi:10.1088/ 0264-9381/31/12/125008. arXiv:1307.5885 [gr-qc]

16. T. Thiemann, Quantum spin dynamics (QSD). Class. Quant. Grav. 15, 839-873 (1998). doi:10.1088/0264-9381/15/4/011. arXiv:gr-qc/9606089 [gr-qc]

17. T. Thiemann, Quantum spin dynamics (QSD): V. Quantum gravity as the natural regulator of matter quantum field theories. Class. Quant. Grav. 15, 1281-1314 (1998). doi:10.1088/0264-9381/15/5/012. arXiv:gr-qc/9705019 [gr-qc]

18. R. De Pietri, C. Rovelli, Geometry eigenvalues and scalar product from recoupling theory in loop quantum gravity. Phys. Rev. D. 54, 2664-2690 (1996). doi:10.1103/PhysRevD.54.2664. arXiv:gr-qc/9602023 [gr-qc]

19. J. Brunnemann, T. Thiemann, Simplification of the spectral analysis of the volume operator in loop quantum gravity. Class. Quant. Grav. 23, 1289-1346 (2006). doi:10.1088/0264-9381/23/4/014. arXiv:gr-qc/0405060 [gr-qc]

20. N.H. Dass, M. Mathur, On loop states in loop quantum gravity. Class. Quant. Grav. 24, 2179-2192 (2007). doi:10.1088/0264-9381/24/9/002. arXiv:gr-qc/0611156 [gr-qc]

21. R. Borissov, R. De Pietri, C. Rovelli, Matrix elements of Thiemann's Hamiltonian constraint in loop quantum gravity. Class. Quant. Grav. 14, 2793-2823 (1997). doi:10.1088/0264-9381/14/10/008. arXiv:gr-qc/9703090 [gr-qc]

22. M. Gaul, C. Rovelli, A generalized Hamiltonian constraint operator in loop quantum gravity and its simplest Euclidean matrix elements. Class. Quant. Grav. 18, 1593-1624 (2001). doi:10.1088/0264-9381/18/9/301. arXiv:gr-qc/0011106 [gr-qc]

23. E. Alesci, K. Liegener, A. Zipfel, Matrix elements of Lorentzian Hamiltonian constraint in loop quantum gravity. Phys. Rev. D 88, 084043 (2013). doi:10.1103/PhysRevD.88.084043. arXiv:1306.0861 [gr-qc]

24. M. Han, Y. Ma, Dynamics of scalar field in polymer-like representation. Class. Quant. Grav. 23, 2741-2760 (2006). doi:10.1088/0264-9381/ 23/7/031. arXiv:gr-qc/0602101 [gr-qc]

25. A. Ashtekar, M. Bojowald, J. Lewandowski, Mathematical structure of loop quantum cosmology. Adv. Theor. Math. Phys. 7, 233-268 (2003). doi:10.4310/ATMP.2003.v7.n2.a2. arXiv:gr-qc/0304074 [gr-qc]

26. B. Gupt, P. Singh, Contrasting features of anisotropic loop quantum cosmologies: The role of spatial curvature. Phys. Rev. D 85, 044011 (2012). doi:10.1103/PhysRevD.85.044011. arXiv:1109.6636 [gr-qc]

27. J. Brunnemann, T. Thiemann, Unboundedness of triad-like operators in loop quantum gravity. Class. Quant. Grav. 23, 1429-1484 (2006). doi:10.1088/0264-9381/23/5/002. arXiv:gr-qc/0505033 [gr-qc]

28. N. Kawamoto, N. Sato, Y. Uchida, 4-dimensional BF gravity on the lattice. Nucl. Phys. B. 574, 809-848 (2000). doi:10.1016/ S0550-3213(00)00017-1. arXiv:hep-th/9911228 [hep-th]

29. E. Alesci, C. Rovelli, Complete LQG propagator: difficulties with the Barrett-Crane vertex. Phys. Rev. D 76, 104012 (2007). doi:10.1103/ PhysRevD.76.104012. arXiv:0708.0883 [gr-qc]

30. A.P. Yutsis, I.B. Levinson, V.V. Vanagas, Mathematical Apparatus of the Theory of Angular Momentum. (Israel Program for Scientific Translation, Jerusalem, 1962) Translated from the Russian by A. Sen and R. N, Sen

31. D.M. Brink, G.R. Satchler, Angular Momentum (Oxford Library of the Physical Sciences, Clarendon Press, 1968)

32. D.A. Varshalovich, A.N. Moskalev, V.K. Khersonsky, Quantum Theory of Angular Momentum: Irreducible Tensors, Spherical Harmonics, Vector Coupling Coefficients, 3nj Symbols (World Scientific, Singapore, 1988)

33. L.H. Kauffman, S.L. Lins, Temperley-Lieb Recoupling Theory and Invariant of 3-Manifolds (Princeton University Press, Princeton, 1994) 
34. E. Alesci, F. Cianfrani, Quantum-reduced loop gravity: cosmology. Phys. Rev. D 87, 083521 (2013). doi:10.1103/PhysRevD.87.083521. arXiv:1301.2245 [gr-qc]

35. E. Alesci, M. Assanioussi, J. Lewandowski, I. Mäkinen, Hamiltonian operator for loop quantum gravity coupled to a scalar field. Phys. Rev. D 91, 124067 (2015). doi:10.1103/PhysRevD.91.124067. arXiv:1504.02068 [gr-qc]

36. J. Yang, Y. Ma, New Hamiltonian constraint operator for loop quantum gravity. Phys. Lett. B. 751, 343-347 (2015). doi:10.1016/j.physletb. 2015.10.062. arXiv:1507.00986 [gr-qc]

37. A. Ashtekar, New Hamiltonian formulation of general relativity. Phys. Rev. D 36, 1587-1602 (1987). doi:10.1103/PhysRevD.36.1587

38. J.F.G. Barbero, Real Ashtekar variables for Lorentzian signature space times. Phys. Rev. D. 51, 5507-5510 (1995). doi:10.1103/PhysRevD. 51.5507. arXiv:gr-qc/9410014 [gr-qc]

39. M. Fecko, Differential Geometry and Lie Groups for Physicists (Cambridge University Press, 2006)

40. A. Ashtekar, J. Lewandowski, D. Marolf, J. Mourao, T. Thiemann, Quantization of diffeomorphism invariant theories of connections with local degrees of freedom. J. Math. Phys. 36, 6456-6493 (1995). doi:10.1063/1.531252. arXiv:gr-qc/9504018 [gr-qc]

41. A.R. Edmonds, Angular Momentum in Quantum Mechanics (Princeton University Press, Princeton, 1974)

42. E.P. Wigner, Group Theory and Its Application to the Quantum Mechanics of Atomic Spectra (Academic Press Inc., New York, 1959)

43. Y. Ding, C. Rovelli, The volume operator in covariant quantum gravity. Class. Quant. Grav. 27, 165003 (2010). doi:10.1088/0264-9381/27/ 16/165003. arXiv:0911.0543 [gr-qc]

44. Y. Ding, C. Rovelli, Physical boundary Hilbert space and volume operator in the Lorentzian new spin-foam theory. Class. Quant. Grav. 27, 205003 (2010). doi:10.1088/0264-9381/27/20/205003. arXiv:1006.1294 [gr-qc]

45. K. Giesel, T. Thiemann, Consistency check on volume and triad operator quantization in loop quantum gravity: I. Class. Quant. Grav. 23, 5667-5692 (2006). doi:10.1088/0264-9381/23/18/011. arXiv:gr-qc/0507036 [gr-qc]

46. K. Giesel, T. Thiemann, Consistency check on volume and triad operator quantization in loop quantum gravity: II. Class. Quant. Grav. 23, 5693-5772 (2006). doi:10.1088/0264-9381/23/18/012. arXiv:gr-qc/0507037 [gr-qc]

47. J. Yang, Y. Ma, Graphical method in loop quantum gravity: I. Derivation of the closed formula for the matrix element of the volume operator. arXiv: 1505.00223 [gr-qc]

48. C. Rovelli, L. Smolin, The physical Hamiltonian in nonperturbative quantum gravity. Phys. Rev. Lett. 72, 446-449 (1994). doi:10.1103/ PhysRevLett.72.446. arXiv:gr-qc/9308002 [gr-qc]

49. N. Bodendorfer, T. Thiemann, A. Thurn, New variables for classical and quantum gravity in all dimensions: III. Quantum theory. Class. Quant. Grav. 30, 045003 (2013). doi:10.1088/0264-9381/30/4/045003. arXiv:1105.3705 [gr-qc]

50. X. Zhang, Y. Ma, Extension of loop quantum gravity to $f(R)$ theories. Phys. Rev. Lett. 106, 171301 (2011). doi:10.1103/PhysRevLett.106. 171301. arXiv:1101.1752 [gr-qc]

51. X. Zhang, Y. Ma, Nonperturbative loop quantization of scalar-tensor theories of gravity. Phys. Rev. D 84, 104045 (2011). doi:10.1103/ PhysRevD.84.104045. arXiv:1107.5157 [gr-qc]

52. M. Bojowald, Loop quantum cosmology: III. Wheeler-Dewitt operators. Class. Quant. Grav. 18, 1055-1070 (2001). doi:10.1088/0264-9381/ 18/6/307. arXiv:gr-qc/0008052 [gr-qc]

53. M. Bojowald, R. Swiderski, Spherically symmetric quantum geometry: Hamiltonian constraint. Class. Quant. Grav. 23, 2129-2154 (2006). doi:10.1088/0264-9381/23/6/015. arXiv:gr-qc/0511108 [gr-qc]

54. X. Zhang, Higher dimensional loop quantum cosmology. Eur. Phys. J. C 76, 395 (2016). doi:10.1140/epjc/s10052-016-4249-8. arXiv: 1506.05597 [gr-qc]

55. X. Zhang, Loop quantum cosmology in 2+1 dimension. Phys. Rev. D 90, 124018 (2014). doi:10.1103/PhysRevD.90.124018. arXiv:1411.5201 [gr-qc]

56. T. Thiemann, Kinematical Hilbert spaces for Fermionic and Higgs quantum field theories. Class. Quant. Grav. 15, 1487-1512 (1998). doi:10. 1088/0264-9381/15/6/006. arXiv:gr-qc/9705021 [gr-qc]

57. J. Brunnemann, T. Thiemann, On (cosmological) singularity avoidance in loop quantum gravity. Class. Quant. Grav. 23, 1395-1428 (2006). doi:10.1088/0264-9381/23/5/001. arXiv:gr-qc/0505032 [gr-qc]

58. J. Yang, Y. Ma, New volume and inverse volume operators for loop quantum gravity. Phys. Rev. D 94, 044003 (2016). doi:10.1103/PhysRevD. 94.044003. arXiv:1602.08688 [gr-qc] 